\title{
Grupos de tranças do espaço projetivo
}

\author{
Vinicius Casteluber Laass
}


SERVIÇO DE PÓS-GRADUAÇÃO DO ICMC-USP

Data de Depósito:

Assinatura:

\title{
Grupos de tranças do espaço projetivo
}

\author{
Vinicius Casteluber Laass
}

Orientador: Prof. Dr. Oziride Manzoli Neto

Dissertação apresentada ao Instituto de Ciências Matemáticas e de Computação - ICMC-USP, como parte dos requisitos para obtenção do título de Mestre em Ciências - Matemática. VERSÃO REVISADA.

USP - São Carlos

Abril / 2011 
Ficha catalográfica elaborada pela Biblioteca Prof. Achille Bassi e Seção Técnica de Informática, ICMC/USP, com os dados fornecidos pelo(a) autor(a)

Laass, Vinicius Casteluber
Grupos de tranças do espaço projetivo / Vinicius
Casteluber Lass, orientador Oziride Manzoli Neto --
São Carlos, 2011 .
80 p.
Dissertação (Mestrado - Programa de Pós-Graduação en
Matemática) -- Instituto de Ciências Matemáticas e
de Computação, Universidade de São Paulo, 2011.
1. Grupos de tranças. 2. Espaço projetivo. 3.
Mspaços de configuraço. 4. Apresentação de grupos. I.
Manzoli Neto, Oziride, orient. II. Título.


Aos meus

irmãos Gabriel

e Diogo. 



\section{Agradecimentos}

Uma vez, quando fazia o ensino médio, no Cefetes de Vitória, fui perguntar a professora Vânia, que era minha professora de matemática, se era verdade que professor de matemática morria de fome. E ela disse, "olhe para mim, eu tenho cara de quem passa fome", e eu ri. Ela falou muito sobre matemática, sobre a graduação dela e como ela gostava de ensinar matemática. Eu me empolguei e resolvi fazer matemática. Mas lembro ela dizendo que um colega dela fez pós - graduação em matemática pura e que isso era coisa de doido. Hoje é minha vez de perguntar, "professora Vânia, eu tenho cara de doido?". Espero que ela não responda sinceramente. Serei eternamente grato a professora Vânia por aquela conversa.

Gostaria de agradecer muito também aos professores da UFES, Ana Claudia e Ricardo, que diferentemente da professora Vânia, não disseram que pós - graduação em matemática era coisa de doido, mas me incentivaram e deram conselhos.

Agradeço ao professor Magno, que durante a sua aula de Estruturas Algébricas, resolveu dar um exemplo de um grupo de construção não trivial. Era um tal de grupo fundamental.

E para completar o time de professores da UFES, agraço muitíssimo ao professor Luiz Fernando, pelos papos e pelo belo curso de Espaços Métricos. Sem sombra de dúvidas foi o curso mais legal que fiz na graduação e que mais me incentivou a procurar saber o que era uma tal de topologia.

Já em São Carlos, conheci o professor Oziride, que me encantou com seus desenhos numa palestra do verão e logo após aceitou me orientar. Obrigado professor Oziride por ter me proposto o tema "Grupos de Tranças". Nas palavras do próprio Oziride, "o tema é duro, mas é divertidíssimo". 
Já nesse "mundo topológico", gostaria de agradecer a todos os participantes do grupo de topologia de São Paulo, pelos encontros, e por ter tido a chance de conhecer pessoas tão gente boa.

Quanto aos amigos, faltam palavras... Andreza, Nelson, Rafael, Carlos, Northon, Alex, Greciane, Jorge, Ferraz. Simplesmente obrigado por vocês existirem. Espero que estes não fiquem com ciúmes, mas seria uma indelicadeza se eu não dedicasse a 3 pessoas, um parágrafo para cada um.

Ao Renato, mais que um amigo, um irmão, obrigado pelo conselhos pessoais e matemáticos.

Ao Apoenã, popular Popô, obrigado pelas conversas de botecos.

A Thaís, que por definição é uoh, que sempre esteve com seu ombro amigo a disposição, muito obrigado.

Meus agradecimentos aos docentes e funcionários da USP - São Carlos, sempre tão atenciosos, e ao CNPq, pelo auxílio financeiro.

Deixei para falar de duas pessoas por último. Gostaria de escrever não um parágrafo, mas várias páginas sobre elas, mas como não é possível... Ao meu pai, Sérgio, e a minha mãe, Dora, minha eterna gratidão por terem se empenhado tanto para me oferecer a melhor educação. Obrigado pelo carinho e por estarem sempre presentes, mesmo que a 1300 quilômetros de distância.

Por fim, agradeço a Deus, por ter me dado a oportunidade de fazer o mestrado e por ter colocado todas as pessoas que citei na minha vida. 
Dada uma superfície $M$, definiremos os grupos de tranças de $M$, denotado por $B_{n}(M)$, geometricamente e usando a noção de espaços de configuração. Mostraremos a equivalência das definições. Na mesma linha de raciocínio, definiremos os grupos de tranças puras de superfícies $P_{n}(M)$. Apresentaremos as propriedades mais importantes dos grupos de tranças do plano e mostraremos que $B_{n}\left(\mathbb{R}^{2}\right)$ injeta em $B_{n}(M)$, para muitas superfícies $M$. Mais detalhadamente, obteremos a apresentação de $B_{n}\left(\mathbb{R P}^{2}\right)$ e $P_{n}\left(\mathbb{R P}^{2}\right)$. 

For a surface $M$, we define the braid groups of $M, B_{n}(M)$, geometricaly and using the notion of configuration spaces. We show the equivalence of these definitions. In the sequence, we define the pure braid group of $M, P_{n}(M)$. We present the most important properties of braid groups of the plane and we show that $B_{n}\left(\mathbb{R}^{2}\right)$ embedds in $B_{n}(M)$, for almost all $M$. In a more detailed fashion, we present $B_{n}\left(\mathbb{R P}^{2}\right)$ and $P_{n}\left(\mathbb{R P}^{2}\right)$. 

1 Pré-Requisitos $\quad 13$

1.1 Fibração e Sequência Exata de Homotopia . . . . . . . . . . . . . . . . 13

1.2 Espaços de Configuração . . . . . . . . . . . . . . . . . . 15

1.3 Método de Reidemeister - Schreier . . . . . . . . . . . . . . . . . 20

1.4 Apresentação de uma extensão . . . . . . . . . . . . . . . . . . 27

1.5 Grupos Dicíclicos . . . . . . . . . . . . . . . . . . . . 29

1.6 Alguns resultados sobre grupos . . . . . . . . . . . . . . . 30

2 Grupos de Tranças de uma Superfície 33

2.1 Introdução . . . . . . . . . . . . . . . . . . . . 33

2.2 Grupos de Tranças Geométrico . . . . . . . . . . . . . . . . . . 33

2.3 Grupos de Tranças e Espaços de Configuração . . . . . . . . . . . . . . 37

3 Grupos de Tranças do Plano $\quad 43$

3.1 Introdução . . . . . . . . . . . . . . . . . . . . . . . . . . . . . 43

3.2 Apresentação de $B_{n}\left(\mathbb{R}^{2}\right) \ldots \ldots \ldots \ldots \ldots$. . . . . . . . . . . 43

3.3 Apresentação de $P_{n}\left(\mathbb{R}^{2}\right) \ldots \ldots \ldots \ldots \ldots \ldots$

$3.4 B_{n}\left(\mathbb{R}^{2}\right)$ como subgrupo de $B_{n}(M) \ldots \ldots \ldots \ldots \ldots$

4 Grupos de Tranças do Plano Projetivo $\quad 57$

4.1 Introdução . . . . . . . . . . . . . . . . . . . . 57

4.2 Propriedades de $G_{n}\left(\mathbb{R P}^{2}\right) \ldots \ldots \ldots \ldots \ldots$. . . . . . . . . 58

4.3 Propriedades de $B_{n}\left(\mathbb{R P}^{2}\right) \ldots \ldots \ldots \ldots$. . . . . . . . . . . 65

4.4 O isomorfismo entre $G_{n}\left(\mathbb{R P}^{2}\right)$ e $B_{n}\left(\mathbb{R P}^{2}\right) \ldots \ldots \ldots$. . . . . . . 69 
5 Grupos de Tranças Puras do Espaço Projetivo 
Os grupos de tranças do plano $\mathbb{R}^{2}$ foram definidos por Artin em [1] e muito estudados em [2] e [3]. Fox, em [4], definiu o grupo de tranças para qualquer espaço topológico, generalizando a definição dada por Artin. Em [5], Birman mostrou que se o espaço topológico em questão for uma variedade diferenciável de dimensão maior ou igual que 3, então a teoria de tranças é de pouco interesse. Por este motivo, os grupos de tranças de superfícies tem sido amplamente estudados.

O grupo de $n$-tranças de uma superfície $M$, denotado por $B_{n}(M)$, surge da seguinte maneira: escolhemos um conjunto $X$ de $n$ pontos distintos de $M$. Em $M \times[0,1]$, ligamos os pontos de $X \times 0$ aos pontos de $X \times 1$ por $n$ caminhos. O produto de tranças é definido por concatenação e re-escalamento. As deformações permitidas são homotopias de cordas individuais de tal modo que, durante o processo, cordas distintas nunca se intersectam. Existe um homomorfismo natural de $B_{n}(M)$ no grupo de permutações de $n$ letras. O núcleo deste homomorfismo é o grupo de $n$-tranças puras de $M$, denotado por $P_{n}(M)$.

O objetivo geral desta dissertação é mostrar algumas diferenças entre o grupo de tranças do plano e o grupo de tranças do espaço projetivo. O trabalho está dividido em cinco capítulos.

No primeiro capítulo, expomos alguns pré-requisitos necessários para uma boa leitura desta dissertação, os quais destacamos Espaços de Configuração, Método de Reidemeister - Schreier e Método de apresentação de uma extensão. É esperado que o leitor tenha uma familiaridade com os conceitos de homotopia, grupo fundamental, espaços de recobrimento e apresentação de grupos.

$\mathrm{Na}$ primeira seção do capítulo 2, faremos a definição formal de uma trança e de uma trança pura de uma superfície. Esta construção engloba a definição de Artin para o caso em que a superfície for o plano. Definiremos os grupos de tranças de uma 
superfície, bem como os grupos de tranças puras. Na seção 2, definiremos os grupos de tranças tais como Fox definiu. Ainda na mesma seção, mostraremos a equivalência de definições.

Nas seções 1 e 2 do capítulo 3, faremos um resumo sobre as apresentações de $B_{n}\left(\mathbb{R}^{2}\right)$ e $P_{n}\left(\mathbb{R}^{2}\right)$, respectivamente. Na seção 3 , mostraremos que $B_{n}\left(\mathbb{R}^{2}\right)$ injeta em $B_{n}(M)$ para um número grande de superfícies.

Nos capítulo 4 e 5, obteremos apresentações dos grupos de tranças do espaço projetivo e dos grupos de tranças puras do espaço projetivo. 


\section{Capítulo}

\section{Pré-Requisitos}

\subsection{Fibração e Sequência Exata de Homotopia}

Generalizando a construção do grupo fundamental de um espaço topólogico, temos os grupos de homotopia de dimensão mais alta. Nesta seção faremos um breve comentário sobre estes grupos e sobre a sequência exata de homotopia de uma fibração. As referências indicadas para este assunto são [12] e [14].

Sejam $I^{0}=\{0,1\}$ e $\partial I^{0}=\{0\}$ e para cada $n \geq 1$, sejam $I^{n}=\underbrace{[0,1] \times \ldots \times[0,1]}_{n \text {-vezes }}$ e $\partial I^{n}$ o bordo de $I^{n}$.

Para cada $n \geq 0$, seja $\mathcal{C}_{n}\left(X, x^{0}\right)=\left\{\alpha:\left(I^{n}, \partial I^{n}\right) \rightarrow\left(X, x^{0}\right) \mid \alpha\right.$ é contínua $\}$, sendo $X$ um espaço topológico e $x^{0}$ um ponto fixado de $X$.

Fixemos $n \geq 1$.

Se $\alpha, \beta \in \mathcal{C}_{n}\left(X, x^{0}\right)$, então a função contínua $\alpha * \beta:\left(I^{n}, \partial I^{n}\right) \rightarrow\left(X, x^{0}\right)$, definida por

$$
\alpha * \beta\left(t_{1}, t_{2}, \ldots, t_{n}\right)=\left\{\begin{array}{l}
\alpha\left(2 t_{1}, t_{2}, \ldots, t_{n}\right), \text { se } 0 \leq t_{1} \leq \frac{1}{2} \\
\beta\left(2 t_{1}-1, t_{2}, \ldots, t_{n}\right), \text { se } \frac{1}{2} \leq t_{1} \leq 1
\end{array}\right.
$$

é chamada o produto de $\alpha$ por $\beta$ e pertence a $\mathcal{C}_{n}\left(X, x^{0}\right)$. 
$\alpha$ é dita equivalente a $\beta$, se existe uma homotopia de pares $H:\left(I^{n} \times[0,1], \partial I^{n} \times\right.$ $[0,1]) \rightarrow\left(X, x^{0}\right)$, tal que $H(t, 0)=\alpha(t)$ e $H(t, 1)=\beta(t)$ e neste caso escrevemos $\alpha \cong \beta$.

$\cong$ é uma relação de equivalência. O conjunto $\mathcal{C}_{n}\left(X, x^{0}\right) / \cong$ com a operação induzida * é um grupo, chamado o grupo de homotopia de dimensão $n$ de $X$ com ponto base $x^{0}$ e denotado por $\pi_{n}\left(X, x^{0}\right)$. Notemos que quando $n=1$, temos o grupo fundamental de $X$ com ponto base $x^{0}$.

Se $f:\left(X, x^{0}\right) \rightarrow\left(Y, y^{0}\right)$ é uma função contínua, então a função $f_{*}: \pi_{n}\left(X, x^{0}\right) \rightarrow$ $\pi_{n}\left(Y, y^{0}\right)$, definida por $f_{*}([\alpha])=[f \circ \alpha]$, é um homomorfismo de grupos, chamado homomorfismo induzido por $f$.

Se $p:\left(E, e^{0}\right) \rightarrow\left(B, b^{0}\right)$ é uma aplicação de recobrimento, então $p_{*}: \pi_{n}\left(E, e^{0}\right) \rightarrow$ $\pi_{n}\left(B, b^{0}\right)$ é injetora para $n=1$ e é isomorfismo para todo $n \geq 2$.

Os grupos de homotopia são invariantes por equivalência de homotopia. Mais precisamente, se $f:\left(X, x^{0}\right) \rightarrow\left(Y, y^{0}\right)$ é uma equivalência de homotopia, então $f_{*}$ : $\pi_{n}\left(X, x^{0}\right) \rightarrow \pi_{n}\left(Y, y^{0}\right)$ é isomorfismo.

Para $n \geq 2, \pi_{n}\left(X, x^{0}\right)$ é grupo abeliano.

Se $x^{0}$ e $x^{1}$ pertencem a mesma componente conexa por caminhos de $X$, então $\pi_{n}\left(X, x^{0}\right)$ e $\pi_{n}\left(X, x^{1}\right)$ são isomorfos para todo $n$.

Se $X$ é um espaço topológico contrátil e $x^{0} \in X$, então $\pi_{n}\left(X, x^{0}\right)$ é trivial para todo $n \geq 1$.

O conjunto $\pi_{0}\left(X, x^{0}\right)$ é definido de modo análogo aos conjuntos $\pi_{n}\left(X, x^{0}\right)$, entranto não tem estrutura de grupo. A cardinalidade do conjunto $\pi_{0}\left(X, x^{0}\right)$ é igual a cardinalidade do conjunto de componentes conexas por caminho de $X$.

Se $f:\left(X, x^{0}\right) \rightarrow\left(Y, y^{0}\right)$ é uma função contínua, então $f_{*}: \pi_{0}\left(X, x^{0}\right) \rightarrow \pi_{0}\left(Y, y^{0}\right)$ dada por $f_{*}([\alpha])=[f \circ \alpha]$ é uma função bem definida, chamada induzida de $f$ em $\pi_{0}$.

Um função contínua e sobrejetora $p: E \rightarrow B$ é dita uma fibração localmente trivial com fibra típica $F$, se para cada $x \in B$, existe uma vizinhança aberta $U \ni x$ e um homeomorfismo $\phi: U \times F \rightarrow p^{-1}(U)$, tal que se $p_{U}: U \times F \rightarrow U$ é a projeção no primeiro fator, então é comutativo o seguinte diagrama:

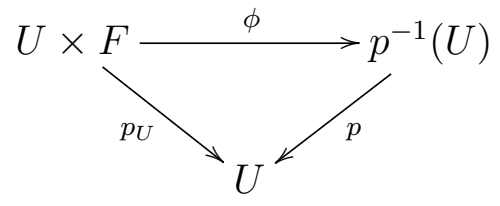

A vizinhança $U$ é dita uma vizinhança distinguida de $x$. O homeomorfismo $\phi$ é chamado de trivialização local. Notemos que $F$ é homeomorfo a $p^{-1}(x)$, para cada cada $x \in B$, logo existe um mergulho $i: F \rightarrow E$. Portanto, podemos considerar $F$ como subespaço de $E$ e $i: F \rightarrow E$ como sendo a inclusão. 
Seja $p:\left(E, e^{0}\right) \rightarrow\left(B, b^{0}\right)$ uma fibração localmente trivial com fibra típica $F$. Então, para todo $n \geq 1$, existe uma função $\partial: \pi_{n}\left(B, b^{0}\right) \rightarrow \pi_{n-1}\left(F, e^{0}\right)$, que é homomorfismo para $n \geq 2$, tal que a seguinte sequência é exata:

$$
\begin{gathered}
\cdots \stackrel{\partial}{\longrightarrow} \pi_{n}\left(F, e^{0}\right) \stackrel{i_{*}}{\longrightarrow} \pi_{n}\left(E, e^{0}\right) \stackrel{p_{*}}{\longrightarrow} \pi_{n}\left(B, b^{0}\right) \stackrel{\partial}{\longrightarrow} \pi_{n-1}\left(F, e^{0}\right) \stackrel{i_{*}}{\longrightarrow} \cdots \\
\cdots \stackrel{p_{*}}{\longrightarrow} \pi_{2}\left(B, b^{0}\right) \stackrel{\partial}{\longrightarrow} \pi_{1}\left(F, e^{0}\right) \stackrel{i_{*}}{\longrightarrow} \pi_{1}\left(E, e^{0}\right) \stackrel{p_{*}}{\longrightarrow} \pi_{1}\left(B, b^{0}\right) \stackrel{\partial}{\longrightarrow} \\
\pi_{0}\left(F, e^{0}\right) \stackrel{i_{*}}{\longrightarrow} \pi_{0}\left(E, e^{0}\right) \stackrel{p_{*}}{\longrightarrow} \pi_{0}\left(B, b^{0}\right)
\end{gathered}
$$

Na ponta final, definimos o núcleo de uma função como sendo o conjunto de todos os elementos que são mandados na classe da função constante.

A sequência acima é chamada sequência exata de homotopia associada a fibração $p$.

A sequência exata de homotopia satisfaz uma condição de naturalidade, no seguinte sentido: sejam $p: E \rightarrow B$ e $p^{\prime}: E^{\prime} \rightarrow B^{\prime}$ fibrações localmente triviais com fibras típicas $F$ e $F^{\prime}$ respectivamente. Sejam $i: F \rightarrow E$ e $i^{\prime}: F^{\prime} \rightarrow E^{\prime}$ as inclusões. Suponhamos que existam aplicações contínuas $f: F \rightarrow F^{\prime}$, e $: E \rightarrow E^{\prime}$ e $b: B \rightarrow B^{\prime}$, tais que tornam comutativo o seguinte diagrama:

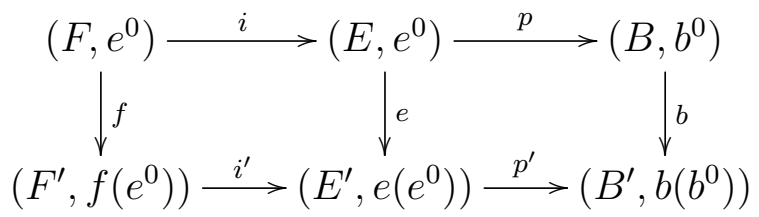

Então, é comutativo o seguinte diagrama (omitimos os pontos bases):

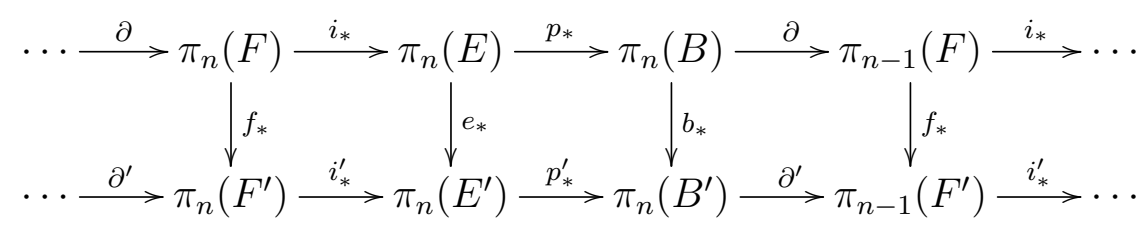

\subsection{Espaços de Configuração}

Definição 1.2.1. Dado um espaço topológico $X$, o conjunto $F_{n}(X)=\left\{\left(x_{1}, \ldots, x_{n}\right) \in\right.$ $X \times \ldots \times X \mid x_{i} \neq x_{j}$ se $\left.i \neq j\right\}$, munido da topologia induzida, é chamado de Espaço de Configuração de $n$ pontos ordenados de $X$. 
Os espaços de configuração são de grande importância na teoria de Tranças, e sendo nosso interesse em grupos de Tranças de Superfícies, fixemos um superfície topológica conexa e sem bordo $\boldsymbol{M}$ e vamos fazer algumas considerações sobre $F_{n}(M)$. Neste caso, $F_{n}(M)$ é uma variedade conexa de dimensão $2 n$. Logo, seus grupos de homotopia independem do ponto base.

Agora, para cada $m$ inteiro não negativo, seja $Q_{m}=\left\{q_{1}, \ldots, q_{m}\right\}$ um conjunto de $m$ pontos distintos de $M$. Notemos que $Q_{0}$ é o conjunto vazio. No estudo dos espaços de configuração é importante considerarmos o espaço

$$
F_{m, n}(M):=F_{n}\left(M-Q_{m}\right)
$$

Notemos que $F_{m, n}(M)$ também é variedade de dimensão $2 n$.

Se $Q_{m}^{\prime}=\left\{q_{1}^{\prime}, \ldots, q_{m}^{\prime}\right\}$ é outro subconjunto de pontos dois a dois distintos de $M$, é bem sabido que existe um homeomorfismo de $M$ que manda $Q_{m}$ em $Q_{m}^{\prime}$. Logo $M-Q_{m}$ é homeomorfo a $M-Q_{m}^{\prime}$ e portanto $F_{n}\left(M-Q_{m}\right)$ é homeomorfo a $F_{n}\left(M-Q_{m}^{\prime}\right)$, ou seja, a escolha do conjunto $Q_{m}$ é irrelevante.

Notemos que $F_{0, n}(M)=F_{n}(M)$ e $F_{m, 1}=\left(M-Q_{m}\right)$.

O próximo teorema é de grande importância no estudo dos Grupos de Tranças.

Teorema 1.2.2 (Fadell e Neuwirth). Suponhamos $n \geq 2$ e $1 \leq r<n$. Então as aplicações

$$
\epsilon, \pi: F_{m, n}(M) \rightarrow F_{m, r}(M)
$$

definidas por

$$
\begin{aligned}
\epsilon\left(x_{1}, \ldots, x_{r}, x_{r+1}, \ldots, x_{n}\right) & =\left(x_{1}, \ldots, x_{r}\right) \\
\pi\left(x_{1}, \ldots, x_{n-r}, x_{n-r+1}, \ldots, x_{n}\right) & =\left(x_{n-r+1}, \ldots, x_{n}\right),
\end{aligned}
$$

são fibrações localmente triviais com fibra típica $F_{m+r, n-r}(M)$.

Prova: Vamos fazer apenas a prova que $\epsilon$ é um fibração localmente trivial. A prova que $\pi$ é fibração localmente trivial é totalmente análoga.

Seja $x^{0}=\left(x_{1}^{0}, \ldots, x_{r}^{0}\right) \in F_{m, r}(M)$. Primeiro vamos mostrar que $\epsilon^{-1}(x)$ é homeomorfo a $F_{m+r, n-r}(M)$.

Seja $Q_{m}=\left\{q_{1}, \ldots, q_{m}\right\}$ o conjunto usado para construir $F_{m, n}(M)$ e $F_{m, r}(M)$. Notemos que $\epsilon^{-1}\left(x^{0}\right)=\left\{\left(x_{1}^{0}, \ldots, x_{r}^{0}, y_{1}, \ldots, y_{n-r}\right) \mid y_{i} \in M-Q_{m}\right.$ e todas as coordenadas são diferentes $\}$.

Ponhamos $Q_{m+r}=Q_{m} \cup\left\{x_{1}^{0}, \ldots, x_{r}^{0}\right\}$. Então

$$
F_{m+r, n-r}(M)=\left\{\left(y_{1}, \ldots, y_{n-r}\right) \mid y_{i} \in M-Q_{m+r} \text { e } y_{i} \neq y_{j} \text { se } i \neq j\right\}
$$


e é claro que aplicação $h: F_{m+r, n-r}(M) \rightarrow \epsilon^{-1}\left(x^{0}\right)$, dada por

$$
h\left(y_{1}, \ldots, y_{n-r}\right)=\left(x_{1}^{0}, \ldots, x_{r}^{0}, y_{1}, \ldots, y_{n-r}\right)
$$

é um homeomorfismo.

Agora vamos mostrar que $\epsilon$ é localmente trivial em torno de $x^{0}$.

Sendo $M$ superfície, então para cada $i \in\{1, \ldots r\}$, existe uma vizinhança $U_{i}$ de $x_{i}^{0}$ com as seguintes propriedades:

(a) $U_{i}$ é homeomorfa ao disco aberto unitário;

(b) $\overline{U_{i}}$ é homeomorfo ao disco fechado unitário;

(c) $\bar{U}_{i} \cap Q_{m}=\emptyset$;

(d) $\overline{U_{k}} \cap \overline{U_{j}}=\emptyset$ se $k \neq j$.

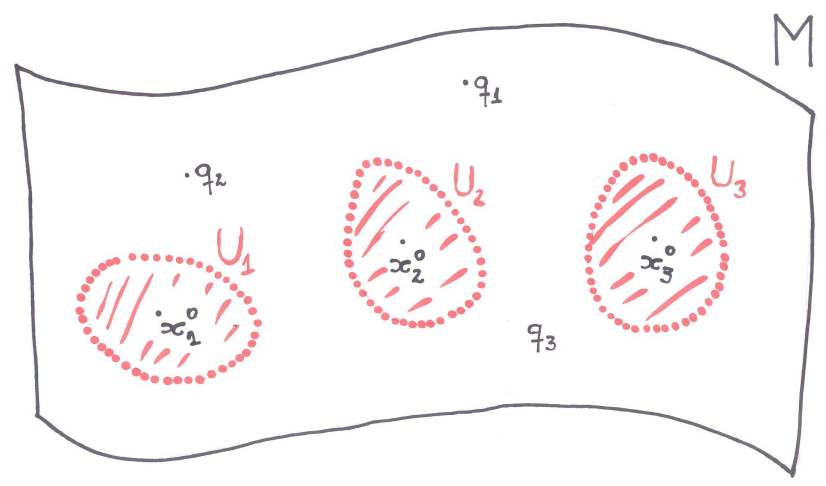

Figura 1.1: os abertos $U_{i}$

Notemos que $U=U_{1} \times \ldots \times U_{r}$ é um subconjunto de $F_{m, r}$ e é uma vizinhança aberta de $x^{0}$.

Para cada $i \in\{1, \ldots, r\}$, seja $\theta_{i}: U_{i} \times \overline{U_{i}} \rightarrow \overline{U_{i}}$ uma aplicação com as seguintes propriedades:

(i) $\theta_{i}^{x}: \overline{U_{i}} \rightarrow \overline{U_{i}}$ dada por $\theta_{i}^{x}(y)=\theta_{i}(x, y)$ é um homeomorfismo que fixa o bordo de $U_{i}$ (que é homeomorfo ao círculo unitário);

(ii) $\theta_{i}^{x}(x)=x_{i}^{0}$.

Na observação a seguir mostramos que tais funções existem.

Pela construção dos $U_{i}$ 's e das $\theta_{i}$ 's, está bem definida a aplicação

$$
\theta: U \times M \rightarrow M
$$

dada por 


$$
\theta\left(x_{1}, \ldots, x_{r}, y\right)=\left\{\begin{array}{l}
\theta_{i}^{x_{i}}(y), \text { se } y \in U_{i} \\
y, \text { se } y \notin U_{1} \cup \ldots \cup U_{r}
\end{array}\right.
$$

Notemos que para cada $x=\left(x_{1}, \ldots, x_{r}\right) \in U$, então a função $\theta^{x}: M \rightarrow M$, definida por $\theta^{x}(y)=\theta\left(x_{1}, \ldots, x_{r}, y\right)$ é um homeomorfismo.

Definimos $\Phi: U \times F_{m+r, n-r}(M) \rightarrow \epsilon^{-1}(U)$ por:

$$
\Phi(\underbrace{x_{1}, \ldots, x_{r}}_{x}, y_{1}, \ldots, y_{n-r})=\left(x_{1}, \ldots, x_{r},\left(\theta^{x}\right)^{-1}\left(y_{1}\right), \ldots,\left(\theta^{x}\right)^{-1}\left(y_{n-r}\right)\right) .
$$

Vamos mostrar que $\Phi$ é bem definida. Primeiro vamos mostrar que as coordenadas de $\Phi\left(x, y_{1}, \ldots, y_{n-r}\right)$ são duas a duas diferentes.

Como $x \in U \subset F_{m, r}(M)$, então $x_{i} \neq x_{j}$ sempre que $i \neq j$;

Se $i \neq j$, então $y_{i} \neq y_{j}$, pois $y_{i}, y_{j} \in F_{m+r, n-r}(M)$ e sendo $\left(\theta^{x}\right)^{-1}$ homeomorfismo, então $\left(\theta^{x}\right)^{-1}\left(y_{i}\right) \neq\left(\theta^{x}\right)^{-1}\left(y_{j}\right)$.

Suponhamos que algum $x_{i}=\left(\theta^{x}\right)^{-1}\left(y_{j}\right)$. Então $\theta^{x}\left(x_{i}\right)=y_{j}$. Mas $x_{i} \in U_{i}$. Logo $\theta^{x}\left(x_{i}\right)=\theta_{i}^{x_{i}}\left(x_{i}\right)=x_{i}^{0}$, pelas definições de $\theta$ e $\theta_{i}^{x_{i}}$ e portanto $x_{i}^{0}=y_{j}$, o que é um absurdo, pois $y_{j} \in M-Q_{m+r}$ e $x_{i}^{0} \in Q_{m+r}$. Segue que $x_{i} \neq\left(\theta^{x}\right)^{-1}\left(y_{j}\right)$.

Agora vamos mostrar que nenhuma das coordenadas de $\Phi$ pertence a $Q_{m}$.

Para todo $i \in\{1, \ldots, r\}, x_{i} \notin Q_{m}$, pois $x=\left(x_{1}, \ldots, x_{r}\right) \in U \subset F_{m, r}(M)$.

Suponhamos que algum $\left(\theta^{x}\right)^{-1}\left(y_{j}\right)=q_{i}$, com $q_{i} \in Q_{m}$, o que implica $y_{j}=\theta^{x}\left(q_{i}\right)$. Como $q_{i} \notin U_{1} \cup \ldots \cup U_{r}$, então $\theta^{x}\left(q_{i}\right)=q_{i}$ e portanto $y_{j}=q_{i}$, o que é um absurdo, pois $y_{j} \in M-Q_{m+r}$ e $Q_{m} \subset Q_{m+r}$.

Agora, definimos $\Psi: \epsilon^{-1}(U) \rightarrow U \times F_{m+1, n-r}(M)=U_{1} \times \ldots \times U_{r} \times F_{m+1, n-r}(M)$ por

$$
\Psi(\underbrace{x_{1}, \ldots, x_{r}}_{x}, y_{1}, \ldots, y_{n-r})=\left(x, \theta^{x}\left(y_{1}\right), \ldots, \theta^{x}\left(y_{r}\right)\right)=\left(x_{1}, \ldots, x_{r}, \theta^{x}\left(y_{1}\right), \ldots, \theta^{x}\left(y_{r}\right)\right)
$$

Por argumentos semelhantes aos anteriores mostra-se que $\Psi$ está bem definida. É fácil ver $\Phi$ e $\Psi$ são inversas uma da outra. Logo são homeomorfismos.

Também é fácil ver que se $p: U \times F_{m+r, n-r}(M) \rightarrow U$ é a projeção em $U$, então o diagrama abaixo é comutativo.

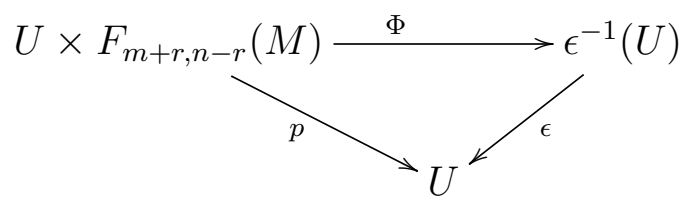

Portanto, $\Phi$ é localmente trivial em torno de $x^{0}$. 


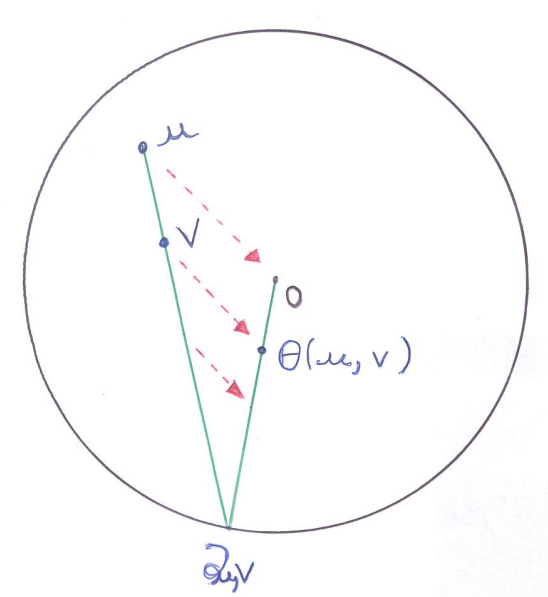

Figura 1.2: função $\theta$

Para mostrar que existem funções $\theta_{i}$ com as propriedades exigidas no teorema, notemos que é suficiente mostrar que existe uma função $\theta$ : $D \times \bar{D} \rightarrow \bar{D}(D$ é o disco unitário aberto do plano) tal que:

(i) Para cada $u \in D, \theta^{u}: \bar{D} \rightarrow \bar{D}$, definida por $\theta^{u}(v)=\theta(u, v)$ é um homeomorfismo que fixa $\partial \bar{D}=\mathbb{S}^{1}$;

(ii) $\theta^{u}(u)=0$ (origem), para todo $u \in D$.

Tal função é construída da seguinte maneira: Seja $(u, v) \in D$. Se $u=v$, colocamos $\theta(u, v)=0$ (a propriedade (ii) já está satisfeita). Se $u \neq v$, então $v-u \neq 0$ e existe um único $t=t(u, v)$ positivo tal que $\partial_{u, v}:=u+t .(v-u) \in S^{1}$. Definimos $\theta(u, v)=\frac{1}{t}$. $\partial_{u, v}$. A figura 1.2 nos mostra a interpetação geométrica da função $\theta$.

Observação 1.2.3. (Recordando) Seja $S_{n}$ o conjunto de todas as bijeções de $\{1, \ldots, n\}$. Se $s, s^{\prime} \in S_{n}$, então definindo o produto $s . s^{\prime}:\{1, \ldots, n\} \rightarrow\{1, \ldots, n\}$ por $s . s^{\prime}(i)=$ $s^{\prime} \circ s(i), S_{n}$ torna-se um grupo com esta operação, chamado Grupo das Permutações de $n$ letras, ou simplesmente, Grupo das Permutações, quando o índice $n$ estiver subtendido. Se $s \in S_{n}$, a notação $\left(\begin{array}{ccc}1 & \ldots & n \\ s(1) & \ldots & s(n)\end{array}\right)$ representa totalmente a função $s$.

Para cada $i=1, \ldots, n-1$, o elemento $s_{i} \in S_{n}$ definido por:

$$
s_{i}(j)=\left\{\begin{array}{l}
j, \text { se } j \neq i, i+1 \\
i, \text { se } j=i+1 \\
i+1, \text { se } j=i
\end{array}\right.
$$

é chamado uma transposição e denotado por $(i, i+1)$. As transposições geram $S_{n}$ e valem as seguintes relações:

(i) $s_{i} . s_{j}=s_{j} . s_{i}$, se $|i-j| \geq 2$;

(ii) $s_{i} . s_{i+1} . s_{i}=s_{i+1} . s_{i} . s_{i+1}$;

(iii) $s_{i}^{2}=I d$.

O próximo teorema é de simples verificação:

Teorema 1.2.4. A funçãoo $\mu: F_{n}(M) \times S_{n} \rightarrow F_{n}(M)$ definida por

$$
\mu\left(x_{1}, \ldots, x_{n}, s\right)=\left(x_{s(1)}, \ldots, x_{s(n)}\right)
$$


é uma ação livre a direita.

Definição 1.2.5. O espaço $C_{n}(M)=F_{n}(M) / S_{n}$, munido da topologia quociente, é chamado o Espaço de Configuração de $n$ pontos não ordenados de $X$.

Corolário 1.2.6. A projeção natural $p: F_{n}(X) \rightarrow C_{n}(X)$ é um recobrimento regular a $n$ !-folhas.

\subsection{Método de Reidemeister - Schreier}

Seja $H$ um subgrupo de $G=\langle X \mid \Re\rangle$. Será possível conseguir uma apresentação de $H$ a partir da apresentação de $G$ ? A resposta é afirmativa se tivermos um conjunto de representantes das classe laterais à esquerda de $H$ em $G$ com um certa propriedade especial, chamada propriedade de Schreier. Este processo é chamado de Método de Reidemeister - Schreier. Nesta seção vamos dar um descrição deste método (incluindo as demonstrações).

Comecemos primeiro com o caso de um subgrupo $L$ de um grupo livre $F(X)$.

Definição 1.3.1. Dizemos que $x=x_{1}^{\epsilon_{1}} \ldots x_{n}^{\epsilon_{n}} \in F(X)$ está escrita na forma reduzida, ou simplesmente, é uma palavra reduzida, se $\epsilon_{i}= \pm 1$ para todo $i=1, \ldots, n$ e $x_{i}^{\epsilon_{i}} x_{i+1}^{\epsilon_{i+1}} \neq$ 1 para todo $i=1, \ldots, n-1$. Se $x=x_{1}^{\epsilon_{1}} \ldots x_{n}^{\epsilon_{n}} \in F(X)$ é uma palavra reduzida, o tamanho de $x$ é, por definição, o número $n$.

Definição 1.3.2. Um conjunto de Schreier de $L$ é um subconjunto $S \subseteq F(X)$, consistindo exatamente de um representante de cada classe lateral à esquerda de $L$ em $F(X)$ com as seguintes propriedades:

(i) O representante da classe $L$ é a palavra vazia 1 ;

(ii) Se $x_{1}^{\epsilon_{1}} \ldots x_{n}^{\epsilon_{n}} \in S$ é uma palavra reduzida, então $x_{2}^{\epsilon_{2}} \ldots x_{n}^{\epsilon_{n}} \in S$.

Dizemos que os elementos de $S$ possuem a propriedade de Schreier.

Segue da definição que se $x_{1}^{\epsilon_{1}} \ldots x_{n}^{\epsilon_{n}} \in S$ é uma palavra reduzida, então $x_{i}^{\epsilon_{i}} x_{i+1}^{\epsilon_{i+1}} \ldots x_{n}^{\epsilon_{n}} \in$ $S$ para todo $i=1, \ldots, n$.

Se $S$ é um conjunto de Schreier de $L$, então dado $x \in F(X)$, denotaremos por $\bar{x} \in S$ o representante da classe $x L$. A pergunta que se põe é: será que sempre existe um conjunto de Schreier de $L$ ? O lema abaixo dá a resposta:

Lema 1.3.3. Se L é um subgrupo de $F(X)$, então existe um conjunto de Schreier de $L$. 
Prova: Dada um classe lateral à esquerda de $L$ em $F(X)$, digamos $S$, chamaremos tamanho de $S$ o tamanho da menor palavra reduzida contida em $S$. A prova é construtiva, usando indução no tamanho das classes. Para a classe $L$, que é a única de tamanho zero, ponhamos 1 como seu representante, ou seja, se $x \in L, \bar{x}=1$. Se $S_{1}$ é uma classe de tamanho 1 , então existe uma palavra reduzida de tamanho $1 \mathrm{em}$ $S_{1}$, digamos $x_{1}^{\epsilon_{1}}$. Ponhamos esta palavra como representante desta classe. Fazemos esta construção para todas as classes de tamanho 1. Se $S_{2}$ é uma classe de tamanho 2 , então existe uma palavra reduzida $x_{1}^{\epsilon_{1}} x_{2}^{\epsilon_{2}} \in S_{2}$. Notemos que $x_{2}^{\epsilon_{2}}$ é uma palavra reduzida de tamanho 1. Logo, pertence a uma classe de tamanho menor ou igual que 1, e portanto, já existe $\overline{x_{2}^{\epsilon_{2}}}$. Pela definição de classe lateral a esquerda, existe $x \in L$, tal que $\overline{x_{2}^{\epsilon_{2}}}=x_{2}^{\epsilon_{2}} x \Rightarrow x_{1}^{\epsilon_{1}} \overline{x_{2}^{\epsilon_{2}}}=x_{1}^{\epsilon_{1}} x_{2}^{\epsilon_{2}} x \Rightarrow x_{1}^{\epsilon_{1}} \overline{x_{2}^{\epsilon_{2}}} \in x_{1}^{\epsilon_{1}} x_{2}^{\epsilon_{2}} L=S_{2}$. Como $\overline{x_{2}^{\epsilon_{2}}}$ é uma palavra reduzida de tamanho menor ou igual a 1 e $x_{1}^{\epsilon_{1}} \overline{x_{2}^{\epsilon_{2}}} \in S_{2}$, então $x_{1}^{\epsilon_{1}} \overline{x_{2}^{\epsilon_{2}}}$ é uma palavra reduzida de tamanho 2. Ponhamos $x_{1}^{\epsilon_{1}} \overline{x_{2}^{\epsilon_{2}}}$ como representante da classe $S_{2}$. Fazemos esta construção para todas as classes de tamanho 2. O processo segue indutivamente e assim obtemos um conjunto de Schreier de L.

Sejam $F(X)$ um grupo livre, $L$ um subgrupo de $F(X)$ e $S$ um conjunto de Schreier de L. Agora, sejam

$$
Y=\{(x, s) \in X \times S \mid \overline{x s} \neq x s\},
$$

$F(Y)$ o grupo livre gerado por $Y$ e $\phi: F(Y) \rightarrow L$ o homomorfismo definido na base por

$$
\phi(x, s)=(\overline{x s})^{-1} x s
$$

e estendido por linearidade.

Notemos que $\phi$ está bem definida. De fato, $(\overline{x s})^{-1} x s L=(\overline{x s})^{-1}(x s L)=(\overline{x s})^{-1}(\overline{x s} L)$ $=(\overline{x s})^{-1} \overline{x s} L=L$.

Teorema 1.3.4 (Nielsen - Schreier). $\phi$ é um isomorfismo entre $L$ e $F(Y)$.

Notemos que o teorema de Nilsen - Schreier fornece uma apresentação para $L$ e uma demonstração que todo subgrupo de um grupo livre é livre. Antes de fazermos a prova, enunciamos o seguinte resultado que será usado muitas vezes na demonstração do teorema:

Lema 1.3.5. Se $a, b \in F(X)$, ent $\tilde{a} o \overline{a \bar{b}}=\overline{a b}$.

Prova: $\bar{b} L=b L \Rightarrow a \bar{b} L=a b L \Rightarrow \overline{a \bar{b}}=\overline{a b}$. 
Prova:(Nielsen - Schreier) A demonstração será construir um homomorfismo $\psi: L \rightarrow$ $F(Y)$ inverso de $\phi$. Seja $x=x_{1}^{\epsilon_{1}} \ldots x_{n}^{\epsilon_{n}} \in L$ escrita na forma reduzida. Para cada $i=1, \ldots, n+1$, definimos

$$
s_{i}=\left\{\begin{array}{l}
\overline{x_{i}^{\epsilon_{i}} \ldots x_{n}^{\epsilon_{n}}}, \text { se } i \neq n+1 \\
1, \text { se } i=n+1
\end{array}\right.
$$

Notemos que $s_{1}=\overline{x_{1}^{\epsilon_{1}} \ldots x_{n}^{\epsilon_{n}}}=\bar{x}=1$, pois $x \in L$.

Se $i<n$, pelo lema 1.3.5, temos $s_{i}=\overline{x_{i}^{\epsilon_{i}} x_{i+1}^{\epsilon_{i+1}} \ldots x_{n}^{\epsilon_{n}}}=\overline{x_{i}^{\epsilon_{i}} \overline{x_{i+1}^{\epsilon_{i+1}} \ldots x_{n}^{\epsilon_{n}}}}=\overline{x_{i}^{\epsilon_{i}} s_{i+1}}$. Esta relação também vale para $i=n$. De fato, $s_{n}=\overline{x_{n}^{\epsilon_{n}}}=\overline{x_{n}^{\epsilon_{n}} 1}=\overline{x_{n}^{\epsilon_{n}} s_{n+1}}$. Portanto

$$
s_{i}=\overline{x_{i}^{\epsilon_{i}} s_{i+1}} \text { para todo } i=1, \ldots, n
$$

Agora, seja $a_{i}=\left(\overline{x_{i}^{\epsilon_{i}} s_{i+1}}\right)^{-1} x_{i}^{\epsilon_{i}} s_{i+1}, i=1, \ldots, n$.

Notemos que

$a_{1} a_{2} \ldots a_{n-1} a_{n}=\left(\overline{x_{1} s_{2}}\right)^{-1} x_{1}^{\epsilon_{1}} s_{2}\left(\overline{x_{2}^{\epsilon_{2}} s_{3}}\right)^{-1} x_{2}^{\epsilon_{2}} s_{3} \ldots\left(\overline{x_{n-1}^{\epsilon_{n-1}} s_{n}}\right)^{-1} x_{n-1}^{\epsilon_{n-1}} s_{n}\left(\overline{x_{n}^{\epsilon_{n}} s_{n+1}}\right)^{-1} x_{n}^{\epsilon_{n}} s_{n+1}$ $\stackrel{(*)}{=} s_{1}^{-1} x_{1}^{\epsilon_{1}} s_{2} s_{2}^{-1} x_{2}^{\epsilon_{2}} s_{3} s_{3}^{-1} \ldots s_{n-1} s_{n-1}^{-1} x_{n-1}^{\epsilon_{n-1}} s_{n} s_{n}^{-1} x_{n}^{\epsilon_{n}} s_{n+1}=x_{1}^{\epsilon_{1}} \ldots x_{n}^{\epsilon_{n}}=x$.

Suponhamos $a_{i} \neq 1$.

Se $\epsilon_{i}=1$, então $1 \neq a_{i}=\left(\overline{x_{i} s_{i+1}}\right)^{-1} x_{i} s_{i+1}=\phi\left(x_{i}, s_{i+1}\right)$.

Se $\epsilon_{i}=-1$, então, usando $\left(^{*}\right)$ e o lema $1.3 .5,1 \neq a_{i}=\left(\overline{x_{i}^{-1} s_{i+1}}\right)^{-1} x_{i}^{-1} s_{i+1}$

$=\left[s_{i+1}^{-1} x_{i} \overline{x_{i}^{-1} s_{i+1}}\right]^{-1}$

$=\left[\left(\overline{s_{i+1}}\right)^{-1} x_{i} s_{i}\right]^{-1}$

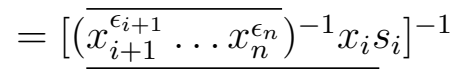

$=\left[\left(\overline{\left(x_{i} x_{i}^{-1} x_{i+1}^{\epsilon_{i+1}} \ldots x_{n}^{\epsilon_{n}}\right.}\right)^{-1} x_{i} s_{i}\right]^{-1}$

$=\left[\left(\overline{x_{i} \overline{x_{i}^{\epsilon_{i}} x_{i+1}^{\epsilon_{i+1}} \ldots x_{n}^{\epsilon_{n}}}}\right)^{-1} x_{i} s_{i}\right]^{-1}$

$=\left[\left(\overline{x_{i} s_{i}}\right)^{-1} x_{i} s_{i}\right]^{-1}=\left[\phi\left(x_{i}, s_{i}\right)\right]^{-1}$.

Observação 1.3.6. Notemos que os argumentos acima mostram que os elementos do conjunto $\left\{(\overline{x s})^{-1} x s \mid x \in X, s \in S\right\}$ geram o grupo $L$.

Definimos $V_{i}=\left\{\begin{array}{l}\left(x_{i}, s_{i+1}\right), \text { se } a_{i} \neq 1, \epsilon_{i}=1 \\ \left(x_{i}, s_{i}\right)^{-1}, \text { se } a_{i} \neq 1, \epsilon_{i}=-1 \\ 1, \text { se } a_{i}=1\end{array}\right.$ e $\psi(x)=V_{1} \ldots V_{n}$.

Vamos dispor todos os elementos utilizados para construir $\psi$ numa tabela, a qual chamamos de tabela de inversão de $x$. 


\begin{tabular}{c|c|c|c|c|c}
$x_{1}^{\epsilon_{1}}$ & $\ldots$ & $x_{i}^{\epsilon_{i}}$ & $\ldots$ & $x_{n}^{\epsilon_{n}}$ & \\
\hline$s_{1}=1$ & $\ldots$ & $s_{i}$ & $\ldots$ & $s_{n}$ & $s_{n+1}=1$ \\
\hline$a_{1}$ & $\ldots$ & $a_{i}$ & $\ldots$ & $a_{n}$ & \\
\hline$V_{1}$ & $\ldots$ & $V_{i}$ & $\ldots$ & $V_{n}$ &
\end{tabular}

Observação 1.3.7. Para definir $\psi$, exigimos que $x=x_{1}^{\epsilon_{1}} \ldots x_{n}^{\epsilon_{n}}$ estivesse na forma reduzida. Entretanto, não é necessário. Suponhamos $x$ escrita da forma

$$
x=x_{1}^{\epsilon_{1}} \ldots x_{i-1}^{\epsilon_{i-1}} x^{\epsilon} x^{-\epsilon} x_{i}^{\epsilon_{i}} \ldots x_{n}^{\epsilon_{n}},
$$

a qual não é reduzida.

Montando a tabela de inversão de $x$ escrita desta última maneira, temos:

\begin{tabular}{c|c|c|c|c|c|c|c|c}
$x_{1}^{\epsilon_{1}}$ & $\ldots$ & $x_{i-1}^{\epsilon_{i-1}}$ & $x^{\epsilon}$ & $x^{-\epsilon}$ & $x_{i}^{\epsilon_{i}}$ & $\ldots$ & $x_{n}^{\epsilon_{n}}$ & \\
\hline$s_{1}=1$ & $\ldots$ & $s_{i-1}$ & $s_{i}$ & $s$ & $s_{i}$ & $\ldots$ & $s_{n}$ & $s_{n+1}=1$ \\
\hline$a_{1}$ & $\ldots$ & $a_{i-1}$ & $a$ & $a^{\prime}$ & $a_{i}$ & $\ldots$ & $a_{n}$ & \\
\hline$V_{1}$ & $\ldots$ & $V_{i-1}$ & $V$ & $V^{\prime}$ & $V_{i}$ & $\ldots$ & $V_{n}$ &
\end{tabular}

$s=\overline{x^{-\epsilon} x_{i}^{\epsilon_{i}} \ldots x_{n}^{\epsilon_{n}}}$

$a=\left(\overline{x^{\epsilon} s}\right)^{-1} x^{\epsilon} s=\left(\overline{x^{\epsilon} \overline{x^{-\epsilon} x_{i}^{\epsilon_{i}} \ldots x_{n}^{\epsilon_{n}}}}\right)^{-1} x^{\epsilon} \overline{x^{-\epsilon} x_{i}^{\epsilon_{i}} \ldots x_{n}^{\epsilon_{n}}}=\left(\overline{x^{\epsilon} x^{-\epsilon} x_{i}^{\epsilon_{i}} \ldots x_{n}^{\epsilon_{n}}}\right)^{-1} x^{\epsilon} \overline{x^{-\epsilon} x_{i}^{\epsilon_{i}} \ldots x_{n}^{\epsilon_{n}}}$

$=\left(\overline{x_{i}^{\epsilon_{i}} \ldots x_{n}^{\epsilon_{n}}}\right)^{-1} x^{\epsilon} \overline{x^{-\epsilon} x_{i}^{\epsilon_{i}} \ldots x_{n}^{\epsilon_{n}}}$

$a^{\prime}=\left(\overline{x^{-\epsilon} s_{i}}\right)^{-1} x^{-\epsilon} s_{i}=\left(\overline{x^{-\epsilon} \overline{x_{i}^{\epsilon_{i}} \ldots x_{n}^{\epsilon_{n}}}}\right)^{-1} x^{-\epsilon} \overline{x_{i}^{\epsilon_{i}} \ldots x_{n}^{\epsilon_{n}}}=\left(\overline{x^{-\epsilon} x_{i}^{\epsilon_{i}} \ldots x_{n}^{\epsilon_{n}}}\right)^{-1} x^{-\epsilon} \overline{x_{i}^{\epsilon_{i}} \ldots x_{n}^{\epsilon_{n}}}$

$\Rightarrow a^{\prime}=a^{-1}$.

Se $a=1$, então $a^{\prime}=1$ e portanto $V=V^{\prime}=1$.

Se $a \neq 1$ e $\epsilon=1$, então $a^{\prime} \neq 1$ e $-\epsilon=-1$. Neste caso, $V=(x, s)$ e $V^{\prime}=(x, s)^{-1}$.

Se $a \neq 1$ e $\epsilon=-1$, então $a^{\prime} \neq 1$ e $-\epsilon=1$. Neste caso, $V=\left(x, s_{i}\right)^{-1}$ e $V^{\prime}=\left(x, s_{i}\right)^{-1}$.

Em todo caso $V^{\prime}=V^{-1}$ e portanto $V_{1} \ldots V_{i-1} V V^{\prime} V_{i} \ldots V_{n}=V_{1} \ldots V_{i-1} V_{i} \ldots V_{n}=$ $\psi(x)$.

Da observação acima, segue que $\psi$ é homomorfismo e da maneira que foi construída, segue que $\phi \circ \psi=I d_{L}$.

Para concluir a demonstração, resta mostrarmos que $\psi \circ \phi=I d_{F(Y)}$. Como $F(Y)$ é livre, basta mostrarmos esta afirmação para os geradores de $F(Y)$, ou seja, os elementos de $Y$. Seja então $(x, s) \in Y \Rightarrow(\overline{x s})^{-1} x s \neq 1$. Sejam $s=x_{1}^{\epsilon_{1}} \ldots x_{n}^{\epsilon_{n}}$ e $\overline{x s}={\tilde{x_{1}}}^{\gamma_{1}} \ldots{\tilde{x_{m}}}^{\gamma_{m}}$ escritas na forma reduzida. Usaremos fortemente o fato que $s$ e $\overline{x s}$ tem a propriedade de Schreier e o lema 1.3.5.

Vamos montar a tabela de inversão $\phi(x, s)=(\overline{x s})^{-1} x s={\tilde{x_{m}}}^{-\gamma_{m}} \ldots{\tilde{x_{1}}}^{-\gamma_{1}} x x_{1}^{\epsilon_{1}} \ldots x_{n}^{\epsilon_{n}}$.

\begin{tabular}{c|c|c|c|c|c|c|c|c|c|c|c}
$\tilde{x_{m}}{ }^{-\gamma_{m}}$ & $\ldots$ & ${\tilde{x_{i}}}^{-\gamma_{i}}$ & $\ldots$ & $\tilde{x}_{1}^{-\gamma_{1}}$ & $x$ & $x_{1}^{\epsilon}$ & $\ldots$ & $x_{j}^{\epsilon_{j}}$ & $\ldots$ & $x_{n}^{\epsilon_{n}}$ & \\
\hline$\tilde{s_{m}}=1$ & $\ldots$ & $\tilde{s_{i}}$ & $\ldots$ & $\tilde{s_{1}}$ & $s^{\prime}$ & $s_{1}$ & $\ldots$ & $s_{j}$ & $\ldots$ & $s_{n}$ & $s_{n+1}=1$ \\
\hline$\tilde{a_{m}}$ & $\ldots$ & $\tilde{a_{i}}$ & $\ldots$ & $\tilde{a_{1}}$ & $a$ & $a_{1}$ & $\ldots$ & $a_{j}$ & $\ldots$ & $a_{n}$ & \\
\hline$\tilde{V_{m}}$ & $\ldots$ & $\tilde{V_{i}}$ & $\ldots$ & $\tilde{V_{1}}$ & $V$ & $V_{1}$ & $\ldots$ & $V_{j}$ & $\ldots$ & $V_{n}$ &
\end{tabular}


$\tilde{s}_{i}=\overline{\tilde{x}_{i}{ }^{-\gamma_{i}} \ldots \tilde{x}_{1}^{-\gamma_{1}} x x_{1}^{\epsilon_{1}} \ldots x_{n}^{\epsilon_{n}}}=\overline{\tilde{x}_{i}^{-\gamma_{i}} \ldots{\tilde{x_{1}}}^{-\gamma_{1}} x \overline{x_{1}^{\epsilon_{1}} \ldots x_{n}^{\epsilon_{n}}}}=\overline{{\tilde{x_{i}}}^{-\gamma_{i}} \ldots{\tilde{x_{1}}}^{-\gamma_{1}} x \bar{s}}=\overline{{\tilde{x_{i}}}^{-\gamma_{i}} \ldots \tilde{x}^{-\gamma_{1}} \overline{x s}}$

$=\overline{{\tilde{x_{i}}}^{-\gamma_{i}} \ldots{\tilde{x_{1}}}^{-\gamma_{1}}{\tilde{x_{1}}}^{\gamma_{1}} \ldots \tilde{x}^{\gamma_{i}} \ldots{\tilde{x_{m}}}^{\gamma_{m}}}=\overline{\tilde{x}_{i+1}^{\gamma_{i+1}} \ldots \tilde{x}^{\gamma_{m}}}=\tilde{x_{i+1}}{ }^{\gamma_{i+1}} \ldots \tilde{x_{m}}{ }^{\gamma_{m}}$

$s^{\prime}=\overline{x x_{1}^{\epsilon_{1}} \ldots x_{n}^{\epsilon_{n}}}=\overline{x s}={\tilde{x_{1}}}^{\gamma_{1}} \ldots{\tilde{x_{m}}}^{\gamma_{m}}$

$s_{j}=\overline{x_{j}^{\epsilon_{j}} \ldots x_{n}^{\epsilon_{n}}}=x_{j}^{\epsilon_{j}} \ldots x_{n}^{\epsilon_{n}}$

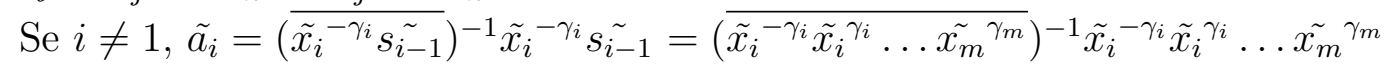

$=\left(\overline{\tilde{x}_{i+1}^{\gamma_{i+1}} \ldots \tilde{x_{m}}{ }^{\gamma_{m}}}\right)^{-1} \tilde{x}_{i+1}^{\gamma_{i+1}} \ldots \tilde{x_{m}}{ }^{\gamma_{m}}=\left(\tilde{x_{i+1}}{ }^{\gamma_{i+1}} \ldots{\tilde{x_{m}}}^{\gamma_{m}}\right)^{-1} \tilde{x}_{i+1}^{\gamma_{i+1}} \ldots{\tilde{x_{m}}}^{\gamma_{m}}=1$

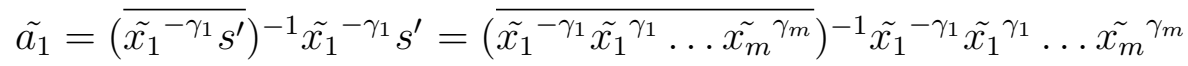

$=\left(\tilde{{\tilde{x_{2}}}^{\gamma_{2}} \ldots{\tilde{x_{m}}}^{\gamma_{m}}}\right)^{-1}{\tilde{x_{2}}}^{\gamma_{2}} \ldots \tilde{x_{m}}{ }^{\gamma_{m}}=\left(\tilde{x_{2}}{ }^{\gamma_{2}} \ldots{\tilde{x_{m}}}^{\gamma_{m}}\right)^{-1}{\tilde{x_{2}}}^{\gamma_{2}} \ldots{\tilde{x_{m}}}^{\gamma_{m}}=1$

$\tilde{V}_{i}=1$, para todo $i=1, \ldots, m$

$a=\left(\overline{x s_{1}}\right)^{-1} x s_{1}=\left(\overline{x x_{1}^{\epsilon_{1}} \ldots x_{n}^{\epsilon_{n}}}\right)^{-1} x x_{1}^{\epsilon_{1}} \ldots x_{n}^{\epsilon_{n}}=(\overline{x s})^{-1} x s \neq 1$

$V=\left(x, s_{1}\right)=\left(x, x_{1}^{\epsilon_{1}} \ldots x_{n}^{\epsilon_{n}}\right)=(x, s)$

$a_{j}=\left(\overline{x_{j}^{\epsilon_{j}} s_{j+1}}\right)^{-1} x_{j}^{\epsilon_{j}} s_{j+1}=\left(\overline{x_{j}^{\epsilon_{j}} x_{j+1}^{\epsilon j+1} \ldots x_{n}^{\epsilon_{n}}}\right)^{-1} x_{j}^{\epsilon_{j}} x_{j+1}^{\epsilon j+1} \ldots x_{n}^{\epsilon_{n}}=\left(x_{j}^{\epsilon_{j}} \ldots x_{n}^{\epsilon_{n}}\right)^{-1} x_{j}^{\epsilon_{j}} \ldots x_{n}^{\epsilon_{n}}=$ 1

$V_{i}=1$, para todo $i=1, \ldots, n$.

Portanto, $\psi(\phi(x, s))=\tilde{V}_{m} \ldots \tilde{V}_{1} V V_{1} \ldots V_{n}=V=(x, s)$ e isto encerra a demonstração.

Agora vamos tratar do caso geral de um subgrupo $H$ de um grupo $G=\langle X \mid \Re\rangle$.

Seja $\lambda: F(X) \rightarrow G$ a projeção natural. Logo, o núcleo de $\lambda$ é o menor subgrupo normal de $F(X)$ que contém as palavras de $\Re$, o qual denotamos por $\left\langle\Re^{F(X)}\right\rangle$. Isto significa que temos a seguinte sequência exata:

$$
1 \longrightarrow\left\langle\Re^{F(X)}\right\rangle \stackrel{i}{\longrightarrow} F(X) \stackrel{\lambda}{\longrightarrow} G \longrightarrow 1
$$

Seja $L=\lambda^{-1}(H) \subset F(X)$. O lema 1.3.3 diz que existe um conjunto de Schreier $S$ de $L$ e o teorema de Nilsen - Schreier fornece um isomorfismo $\phi: F(Y) \rightarrow L$. Definimos $\mu=\lambda \circ \phi: F(Y) \rightarrow H$. Temos então o seguinte diagrama comutativo, cuja sequência na horizontal é exata:

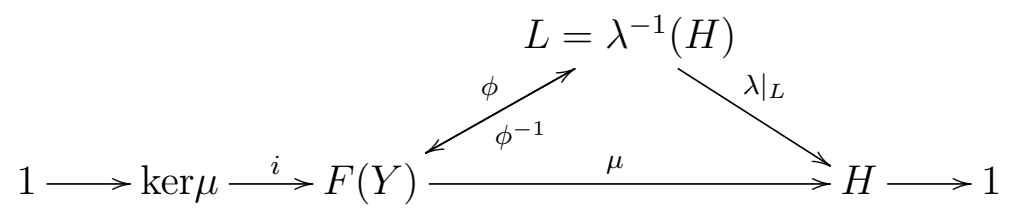

Se encontrarmos um subconjunto $\Im \subset F(Y)$, tal que o menor subgrupo normal contendo $\Im$ seja igual a $\operatorname{ker} \mu$, então teremos uma apresentação para $H$.

Seja então $v \in \operatorname{ker} \mu \Rightarrow \phi(v) \in \operatorname{ker} \lambda=\left\langle\Re^{F(X)}\right\rangle$. Logo

$$
\phi(v)=\left(u_{1}^{-1} r_{1}^{\epsilon_{1}} u_{1}\right) \ldots\left(u_{n}^{-1} r_{n}^{\epsilon_{n}} u_{n}\right)
$$


$\operatorname{com} u_{i} \in F(X), r_{i} \in \Re$ e $\epsilon_{i}= \pm 1$.

Cada $u_{i}$ pertence a alguma classe lateral à esquerda de $L$ em $F(X), \operatorname{logo} u_{i}=s_{i} \tilde{u}_{i}$ para algum $\tilde{u}_{i} \in L$ e para algum $s_{i} \in S$ e portanto

$\phi(v)=\left[\left(s_{1} \tilde{u_{1}}\right)^{-1} r_{1}^{\epsilon_{1}} s_{1} \tilde{u_{n}}\right] \ldots\left[\left(s_{n} \tilde{u_{n}}\right)^{-1} r_{n}^{\epsilon_{n}} s_{n} \tilde{u_{n}}\right]=\left[{\tilde{u_{1}}}^{-1}\left(s_{1}^{-1} r_{1}^{\epsilon_{1}} s_{1}\right) \tilde{u}_{1}\right] \ldots\left[\tilde{u}_{n}^{-1}\left(s_{n}^{-1} r_{n}^{\epsilon_{n}} s_{n}\right) \tilde{u_{n}}\right]$

Cada $\left(s_{i}^{-1} r_{i} s_{i}\right) \in \operatorname{ker} \lambda$ e como $\operatorname{ker} \lambda \subseteq L$, então podemos aplicar $\phi^{-1}$ em $\left(s_{i}^{-1} r_{i} s_{i}\right)$. Colocando $v_{i}=\phi^{-1}\left(\tilde{u}_{i}\right)$, então

$$
v=v_{1}^{-1}\left[\phi^{-1}\left(s_{1}^{-1} r_{1} s_{1}\right)\right]^{\epsilon_{1}} v_{1} \ldots v_{n}^{-1}\left[\phi^{-1}\left(s_{n}^{-1} r_{n} s_{n}\right)\right]^{\epsilon_{n}} v_{n} .
$$

Isto mostra que se definirmos $\Im=\left\{\phi^{-1}\left(s^{-1} r s\right) \mid s \in S, r \in \Re\right\}$, então $\operatorname{ker} \mu \subset$ $\left\langle\Im^{F(Y)}\right\rangle$, sendo $\left\langle\Im^{F(Y)}\right\rangle$ o menor subgrupo normal de $F(Y)$ que contém $\Im$.

Agora se $q \in \Im$, então $q=\phi^{-1}\left(s^{-1} r s\right)$, para algum $s \in S$ e algum $r \in \Re$. Então

$$
\mu(q)=\lambda\left(\phi\left(\phi^{-1}\left(s^{-1} r s\right)\right)\right)=\lambda\left(s^{-1} r s\right)=1 .
$$

Logo $q \in \operatorname{ker} \mu$, o que mostra que $\Im \subset \operatorname{ker} \mu$ e como ker $\mu$ é subgrupo normal de $F(Y)$, então $\left\langle\Im^{F(Y)}\right\rangle \subset \operatorname{ker} \mu$.

Isto mostra o:

Teorema 1.3.8 (Reidemeister - Schreier). O subgrupo H pode ser apresentado como $\langle Y \mid \Im\rangle$, sendo $\Im=\left\{\phi^{-1}\left(s^{-1} r s\right) \mid s \in S, r \in \Re\right\}$.

Vamos transformar todo este arcabouço teórico apresentado até aqui num método mais prático, justificando cada passagem.

Como $\phi$ é isomorfismo, então $H=F(Y) /\langle\Im\rangle \approx L / \phi(\langle\Im\rangle) \approx L /\langle\phi(\Im)\rangle$. Agora $\phi(\Im)=\left\{s^{-1} r s \mid s \in S, r \in \Re\right\}$, pela definição do conjunto $\Im$.

Pela observação 1.3.6, o conjunto $\left\{(\overline{x s})^{-1} x s \mid x \in X, s \in S\right\}$ gera L. Portanto, para obter uma nova apresentação de $H$ resta apenas escrever cada elemento de $\phi(\Im)$ em termos destes geradores de $L$. Mas isto é feito também usando a observação 1.3.6.

É possível simplificar ainda mais este processo.

Suponhamos $x L=x^{\prime} L$. Logo, existe $l \in L$, tal que $x=x^{\prime} l \Rightarrow \lambda(x)=\lambda\left(x^{\prime}\right) \lambda(l)$. Como $L=\lambda^{-1}(H)$, então $\lambda(l) \in H$ e portanto $\lambda(x) H=\lambda\left(x^{\prime}\right) H$. Isto mostra que está bem definida a função

$$
\begin{gathered}
\tilde{\lambda}: F(X) / L \rightarrow G / H \\
(x L) \mapsto \lambda(x) H
\end{gathered}
$$


Por argumentos semelhantes, mostra-se que esta função é uma bijeção, logo $[F(X)$ : $L]=[G: H]$.

Podemos então definir um conjunto de Schreier de $H$ da seguinte maneira:

Definição 1.3.9. Dizemos que $M \subset G$ é um conjunto de Schreier de $H$ se $M=\lambda(S)$, sendo $S$ um conjunto de Schreier de L.

Como $\tilde{\lambda}$ é bijeção, então a cardinalidade de $M$ é igual a cardinalidade de $S$ e o conjunto $M$ é um sistema completo de representantes das classes laterais à esquerda de $H$ em $G$.

Se $g \in G$, também denotamos por $\bar{g}$ o elemento de $M$ que representa a classe lateral $g H$.

Temos então o seguinte resultado:

Lema 1.3.10. Se $x \in F(X)$, então $\lambda(\bar{x})=\overline{\lambda(x)}$.

Prova: Seja $x \in F(X)$. Como $\bar{x} L=x L$ e $\tilde{\lambda}$ é bem definida, então $\lambda(\bar{x}) H=\lambda(x) H$, ou seja, $\lambda(\bar{x})$ e $\lambda(x)$ pertencem a mesma classe lateral a esquerda e portanto $\overline{\lambda(\bar{x})}=\overline{\lambda(x)}$. Mas $\lambda(\bar{x}) \in M, \operatorname{logo} \overline{\lambda(\bar{x})}=\lambda(\bar{x})$. Segue o resultado.

Vale o seguinte resultado, totalmente análogo ao lema 1.3.5.

Lema 1.3.11. Se $a, b \in G$, então $\overline{a \bar{b}}=\overline{a b}$.

Vamos mostrar como os lemas 1.3 .10 e 1.3.11 facilitam os cálculos para achar a apresentação de $H$.

Sejam $s=x_{1}^{\epsilon_{1}} \ldots x_{n}^{\epsilon_{n}} \in S$ e $r=\tilde{x}_{1}^{\gamma_{1}} \ldots \tilde{x}_{n}^{\gamma_{n}} \in \Re$ palavras reduzidas. Então $s^{-1} r s \in$ $\phi(\Im)$. Vamos montar a tabela de inversão de $s^{-1} r s$.

\begin{tabular}{c|c|c|c|c|c|c|c|c|c|c|c|c|c|c|c}
$x_{n}^{-\epsilon_{n}}$ & $\ldots$ & $x_{i}^{-\epsilon_{i}}$ & $\ldots$ & $x_{1}^{-\epsilon_{1}}$ & $\tilde{x}_{1}^{\gamma_{1}}$ & $\ldots$ & $\tilde{x}_{j}^{\gamma_{j}}$ & $\ldots$ & $\tilde{x}_{m}^{\gamma_{m}}$ & $x_{1}^{\epsilon_{1}}$ & $\ldots$ & $x_{i}^{\epsilon_{i}}$ & $\ldots$ & $x_{n}^{\epsilon_{n}}$ & \\
\hline$s_{n}$ & $\ldots$ & $s_{i}$ & $\ldots$ & $s_{1}$ & $r_{1}$ & $\ldots$ & $r_{j}$ & $\ldots$ & $r_{m}$ & $s_{1}^{\prime}$ & $\ldots$ & $s_{i}^{\prime}$ & $\ldots$ & $s_{n}^{\prime}$ & $s_{n+1}^{\prime}=1$ \\
\hline$a_{n}$ & $\ldots$ & $a_{i}$ & $\ldots$ & $a_{1}$ & $b_{1}$ & $\ldots$ & $b_{j}$ & $\ldots$ & $b_{m}$ & $a_{1}^{\prime}$ & $\ldots$ & $a_{i}^{\prime}$ & $\ldots$ & $a_{n}^{\prime}$ &
\end{tabular}

$s_{i}=\overline{x_{i}^{-\epsilon_{i}} \ldots x_{1}^{-\epsilon_{1}} \tilde{x}_{1}^{\gamma_{1}} \ldots \tilde{x}_{n}^{\gamma_{n}} x_{1}^{\epsilon_{1}} \ldots x_{n}^{\epsilon_{n}}} \stackrel{1.3 .10}{=} \overline{\lambda\left(x_{i}^{-\epsilon_{i}} \ldots x_{1}^{-\epsilon_{1}} \tilde{x}_{1}^{\gamma_{1}} \ldots \tilde{x}_{n}^{\gamma_{n}} x_{1}^{\epsilon_{1}} \ldots x_{n}^{\epsilon_{n}}\right)}$

$=\overline{\lambda\left(x_{i}^{-\epsilon_{i}} \ldots x_{1}^{-\epsilon_{1}}\right) \lambda(r) \lambda\left(x_{1}^{\epsilon_{1}} \ldots x_{n}^{\epsilon_{n}}\right)} \stackrel{\lambda(r)=1}{=} \overline{\lambda\left(x_{i}^{-\epsilon_{i}} \ldots x_{1}^{-\epsilon_{1}} x_{1}^{\epsilon_{1}} \ldots x_{n}^{\epsilon_{n}}\right)} \stackrel{1.3 .10}{=} \overline{x_{i+1}^{\epsilon_{i+1}} \ldots x_{n}^{\epsilon_{n}}} \stackrel{s \in S}{=}$ $x_{i+1}^{\epsilon_{i+1}} \ldots x_{n}^{\epsilon_{n}}$

$a_{i}=\left(\overline{x_{i}^{-\epsilon_{i}} s_{i-1}}\right)^{-1} x_{i}^{-\epsilon_{i}} s_{i-1}=\left(\overline{x_{i}^{-\epsilon_{i}} x_{i}^{\epsilon_{i}} x_{i+1}^{\epsilon_{i+1}} \ldots x_{n}^{\epsilon_{n}}}\right)^{-1} x_{i}^{-\epsilon_{i}} x_{i}^{\epsilon_{i}} x_{i+1}^{\epsilon_{i+1}} \ldots x_{n}^{\epsilon_{n}}=\left(\overline{x_{i+1}^{\epsilon_{i+1}} \ldots x_{n}^{\epsilon_{n}}}\right)^{-1} x_{i+1}^{\epsilon_{i+1}} \ldots x_{n}^{\epsilon_{n}}$ $\stackrel{s \in S}{=}\left(x_{i+1}^{\epsilon_{i+1}} \ldots x_{n}^{\epsilon_{n}}\right)^{-1} x_{i+1}^{\epsilon_{i+1}} \ldots x_{n}^{\epsilon_{n}}=1$. 
$s_{i}^{\prime}=\overline{x_{i}^{\epsilon_{i}} \ldots x_{n}^{\epsilon_{n}}} \stackrel{s \in S}{=} x_{i}^{\epsilon_{i}} \ldots x_{n}^{\epsilon_{n}}$

$a_{i}^{\prime}=\left(\overline{x_{i}^{\epsilon_{i}} s_{i+1}^{\prime}}\right)^{-1} x_{i}^{\epsilon_{i}} s_{i+1}^{\prime}=\left(\overline{x_{i}^{\epsilon_{i}} x_{i+1}^{\epsilon_{i+1}} \ldots x_{n}^{\epsilon_{n}}}\right)^{-1} x_{i}^{\epsilon_{i}} x_{i+1}^{\epsilon_{i+1}} \ldots x_{n}^{\epsilon_{n}} \stackrel{s \in S}{=}\left(x_{i}^{\epsilon_{i}} \ldots x_{n}^{\epsilon_{n}}\right)^{-1} x_{i}^{\epsilon_{i}} \ldots x_{n}^{\epsilon_{n}}=$ 1 .

Portanto, só precisamos calcular os $r_{j}$ 's e os $b_{j}$ 's.

\subsection{Apresentação de uma extensão}

Consideremos a seguinte sequência exata de grupos e homomorfismos:

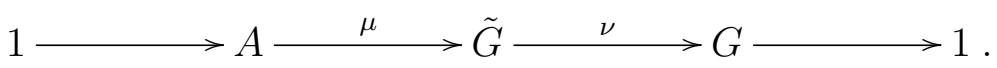

Neste caso, $\tilde{G}$ é chamado uma extensão de $G$ por $A$.

Suponhamos que $A$ e $G$ admitam, respectivamente, as apresentações $\langle Y \mid \mathcal{S}\rangle$ e $\langle X \mid \mathcal{R}\rangle$. Então, a sequência acima pode ser reescrita da seguinte maneira:

$$
1 \longrightarrow\langle Y \mid \mathcal{S}\rangle \stackrel{\mu}{\longrightarrow} \tilde{G} \stackrel{\nu}{\longrightarrow}\langle X \mid \mathcal{R}\rangle \longrightarrow 1
$$

Nesta seção, vamos mostrar que é possível obter uma apresentação de $\tilde{G}$ a partir das apresentações de $A$ e $G$.

Seja

$$
\tilde{Y}=\{\tilde{y}:=\mu(y) \mid y \in Y\}
$$

e para cada $s \in \mathcal{S}$, seja $\tilde{s}$ a palavra obtida de $s$ substituindo cada gerador $y \in Y$ por $\tilde{y} \in \tilde{Y}$. Notemos que como $\mu$ é homomorfismo, cada $\tilde{s}$ representa o elemento neutro em $\tilde{G}$. Seja

$$
\tilde{\mathcal{S}}=\{\tilde{s} \mid s \in \mathcal{S}\}
$$

Como a imagem de $\mu$ é igual ao núcleo de $\nu$, então para cada $x \in X$, existe $\tilde{x} \in \tilde{G}-\operatorname{Im} \mu$, tal que $\nu(\tilde{x})=x$. Seja

$$
\tilde{X}=\{\tilde{x} \mid x \in X\}
$$

Agora, para cada palavra $r \in \mathcal{R}$, seja $\tilde{r}$ a palavra obtida de $r$ trocando cada gerador $x \in X$ por $\tilde{x} \in \tilde{X}$. Novamente, notemos que $\tilde{r} \in$ Ker $\nu$, já que $\nu$ é homomorfismo. Como $\operatorname{Ker} \nu=\operatorname{Im} \mu$, então para cada $\tilde{r}$, existe $v_{r} \in \tilde{G}$, que é uma palavra escrita em termos dos elementos de $\tilde{Y}$, tal que $\tilde{r}=v_{r}$. Seja

$$
\tilde{\mathcal{R}}=\left\{\tilde{r} v_{r}^{-1} \mid r \in \mathcal{R}\right\} .
$$


Desde que $\operatorname{Im} \mu=\operatorname{Ker} \nu$ e Ker $\nu$ é um subgrupo normal de $\tilde{G}$, cada conjugado $\tilde{x}^{-1} \tilde{y} \tilde{x}$ pertence a imagem de $\mu$. Logo, existe uma palavra, digamos $w_{x, y}$, escrita em termos de $\tilde{y}$, tal que $\tilde{x}^{-1} \tilde{y} \tilde{x}=w_{x, y}$. Seja

$$
\tilde{\mathcal{T}}=\left\{\tilde{x}^{-1} \tilde{y} \tilde{x} w_{x, y}^{-1} \mid x \in X, y \in Y\right\} .
$$

Com todos estes conjuntos em mãos, temos o

Teorema 1.4.1. $\tilde{G}$ é isomorfo ao grupo $\langle\tilde{X}, \tilde{Y} \mid \tilde{\mathcal{R}}, \tilde{\mathcal{S}}, \tilde{\mathcal{T}}\rangle$.

Prova: Seja $D$ o grupo definido através da apresentação (1). Consideremos a associação

$$
\begin{gathered}
\theta: D \rightarrow \tilde{G} \\
\tilde{x} \mapsto \tilde{x} \quad \tilde{y} \mapsto \tilde{y}
\end{gathered}
$$

e estendida por lineariadade. Como todas as relações de $D$ valem em $\tilde{G}$, via a associação $\theta$, então $\theta$ é um homomorfismo bem definido. Vamos mostrar que $\theta$ é um isomorfismo. A restrição do homomorfismo $\theta$ ao subgrupo gerado por $\tilde{Y}$, induz um bem definido homomorfismo

$$
\begin{gathered}
\theta_{1}:\langle\tilde{Y}\rangle \rightarrow \operatorname{Im} \mu \approx A \\
\tilde{y} \mapsto y
\end{gathered}
$$

Como todas as relações de $\mathcal{S}$ valem em $\langle\tilde{Y}\rangle$, via a associação $y \mapsto \tilde{y}$, então $\theta_{1}$ é um isomorfismo. A presença das relações $\tilde{\mathcal{T}}$ em $D$ implica que $\langle\tilde{Y}\rangle$ é um subgrupo normal de $D$ e desde que $\theta(\langle\tilde{Y}\rangle) \subset \operatorname{Im} \nu=\operatorname{Ker} \mu$, então $\theta$ induz um bem definido homomorfismo

$$
\begin{gathered}
\theta_{2}: D /\langle\tilde{Y}\rangle \rightarrow \tilde{G} / \operatorname{Im} \mu \approx G \\
{[\tilde{x}] \mapsto x}
\end{gathered}
$$

A presença das relações $\tilde{\mathcal{R}}$ implica que é bem definido o homorfismo

$$
\begin{aligned}
\overline{\theta_{2}}: G & \rightarrow D /\langle\tilde{Y}\rangle \\
x & \mapsto[\tilde{x}]
\end{aligned}
$$

e é claro que $\overline{\theta_{2}}=\theta_{2}^{-1}$. Da maneira como foram construídos os homomorfismos $\theta, \theta_{1}$ e $\theta_{2}$, segue que é comutativo o seguinte diagrama:

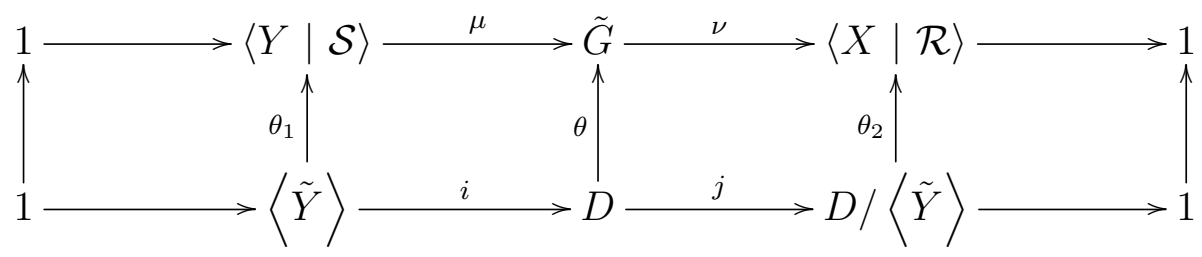

sendo $i$ e $j$, respectivamente, a inclusão e a projeção natural. Como as sequências na horizontal são exatas e $\theta_{1}$ e $\theta_{2}$ são isomorfismos, pelo lema dos cinco, $\theta$ é isomorfismo 


\subsection{Grupos Dicíclicos}

Seja $m>1$. No conjunto de $4 m$ elementos

$$
A=\left\{1, x, \ldots, x^{2 m-1}, y, x y, \ldots, x^{2 m-1} y\right\}
$$

definimos a seguinte operação

1 é o elemento neutro, $x^{a} x^{b}=x^{a+b}, x^{a}\left(x^{b} y\right)=x^{a+b} y,\left(x^{a} y\right) x^{b}=x^{a-b} y$,

$$
\left(x^{a} y\right)\left(x^{b} y\right)=x^{a-b+m} y
$$

sendo $0 \leq a, b \leq 2 m-1$ e as potências de $x$ consideradas módulo $2 m$.

Com argumentos combinatórios, prova-se que $A$, munido da operação acima, é um grupo, chamado Grupo Díciclico de ordem $4 \mathrm{~m}$.

Observemos que $x^{2 m}=x^{0}=1$, yy $=\left(x^{0} y\right)\left(x^{0} y\right)=x^{0-0+m}=x^{m}$ e yyyy $=$ $($ yy $)(y y)=x^{m} x^{m}=x^{2 m}=1 \Rightarrow y^{-1}=y y y=y(y y)=y x^{m}$.

Uma outra construção do grupo dicíclico de ordem $4 m$ é a seguinte:

Considere o grupo cíclico de ordem $2 m, \mathbb{Z}_{2 m}=\left\langle s \mid s^{2 m}=1\right\rangle$. O grupo dicíclico de ordem $4 m$, denotado por $D i c_{4 m}$, é construído adicionando a $\mathbb{Z}_{2 m}$ mais um gerador de ordem 4, digamos $t$, e as relações $t^{2}=s^{m}$ e $t^{-1} s t=s^{-1}$, ou seja,

$$
D i c_{4 m}=\left\langle s, t \mid s^{2 m}=1, t^{2}=s^{m}(*), t^{-1} s t=s^{-1}(* *)\right\rangle
$$

A primeira relação é consequência das outras duas. De fato, $s^{m} \stackrel{(*)}{=} t^{2}=t^{-1} t^{2} t \stackrel{(*)}{=}$ $t^{-1} s^{m} t=\left(t^{-1} s t\right) \stackrel{m}{\stackrel{(* *)}{=}} s^{-m} \Rightarrow s^{2 m}=1$. Portanto

$$
\operatorname{Dic}_{4 m}=\left\langle s, t \mid t^{2}=s^{m}, t^{-1} s t=s^{-1}\right\rangle .
$$

Vamos mostrar que as duas definições coincidem, isto é, $D i c_{4 m} \approx A$.

Consideremos o homomorfismo $\eta: D i c_{4 m} \rightarrow A$, definido na base por $\eta(s)=x$ e $\eta(t)=y$ e estendido por linearidade. Pelo que foi observado, $\eta$ é bem definido.

Agora consideremos $\nu: A \rightarrow D i c_{4 m}$, definido por $\nu\left(x^{c}\right)=s^{c}$ e $\nu\left(x^{d} y\right)=s^{d} t$, $0 \leq c, d \leq 2 m-1$. Usando as relações de $D i c_{4 m}$, mostra-se que $\nu$ é homomorfismo de grupos.

É claro que $\eta$ e $\nu$ são homomorfismos inversos um do outro.

Um outra apresentação para $D i c_{4 m}$ é $B=\left\langle r, v \mid r^{2}=v^{2}=\left(r^{-1} v\right)^{m}\right\rangle$. Para mostrar isto, consideremos os homomorfismos que são definidos na base da seguinte maneira: 


$$
\begin{aligned}
\varphi: B & \rightarrow \text { Dic }_{4 m} \\
r & \mapsto t s^{-1} \\
v & \mapsto t
\end{aligned}
$$

$$
\begin{gathered}
\psi: D i c_{4 m} \rightarrow B \\
s \mapsto r^{-1} v \\
t \mapsto v
\end{gathered}
$$

Notemos que

$$
\begin{gathered}
\varphi\left(r^{2}\right)=\left(t s^{-1}\right)^{2}=t s^{-1}\left(t s^{-1}\right)=t s^{-1} s t=t^{2}=\varphi\left(v^{2}\right) \\
\varphi\left[\left(r^{-1} v\right)^{m}\right]=\left(s t^{-1} t\right)^{m}=s^{m}=t^{2}=\varphi\left(v^{2}\right) \\
\psi\left(t^{2}\right)=v^{2}=\left(r^{-1} v\right)^{m}=\psi\left(s^{m}\right) \\
\psi\left(t^{-1} s t\right)=v^{-1} r^{-1} v v=v^{-1} r^{-1} v^{2}=v^{-1} r^{-1} r^{2}=v^{-1} r=\psi\left(s^{-1}\right) .
\end{gathered}
$$

Isto mostra que $\varphi$ e $\psi$ estão bem definidas e é fácil ver que $\varphi=\psi^{-1}$.

Vamos mostrar que para $m=2, D i c_{4.2}=D i c_{8} \approx \mathcal{Q}_{8}$, sendo $\mathcal{Q}_{8}$ o grupo dos quatérnios.

Consideremos $\mathcal{Q}_{8}=\{ \pm 1, \pm i, \pm j, \pm k\}$ com a regra usual de operação $i^{2}=j^{2}=k^{2}=$ $i j k=-1$.

Definimos $\mu: \mathcal{Q}_{8} \rightarrow\left\langle r, v \mid r^{2}=v^{2}=\left(r^{-1} v\right)^{2}\right\rangle \approx D i c_{8}$, da seguinte maneira:

$$
1 \mapsto 1,-1 \mapsto r^{2}, \pm i \mapsto r^{ \pm 1}, \pm j \mapsto r v^{-1}, \pm k \mapsto v^{ \pm 1}
$$

Usando as relações de $\left\langle r, v \mid r^{2}=v^{2}=\left(r^{-1} v\right)^{2}\right\rangle$, mostra-se que $\mu$ é homomorfismo.

Agora definimos $\delta:\left\langle r, v \mid r^{2}=v^{2}=\left(r^{-1} v\right)^{2}\right\rangle \rightarrow \mathcal{Q}_{8}$ na base por

$$
\delta(r)=i, \delta(v)=k
$$

e estendemos por linearidade.

É fácil mostrar que $\delta$ está bem definida e que $\delta=\mu^{-1}$.

\subsection{Alguns resultados sobre grupos}

Lema 1.6.1. Se $F:=F\left(x_{1}, \ldots, x_{n}\right)$ é um grupo livre de rank $n>1$, então $F$ é isomorfo a $\mathcal{I}_{F}$, sendo $\mathcal{I}_{F}$ o grupo dos automorfismo internos de $F$.

Prova: Consideremos o homomorfismo $\mathcal{I}: F \rightarrow \mathcal{I}_{F}$ que associa $x_{i}$ ao automorfismo interno

$$
\begin{aligned}
& \mathcal{I}_{x_{i}}: F \rightarrow F \\
& x \mapsto x_{i} x x_{i}^{-1}
\end{aligned}
$$


e estendido por lineariadade. Vamos mostrar que $\mathcal{I}$ é isomorfismo, ou equivalentemente, que $\mathcal{I}$ é bijeção. Claramente $\mathcal{I}$ é sobrejetora. Seja $a \in F$ tal que $\mathcal{I}_{a}=I d$. Então, para todo $x \in F, a x a^{-1}=x \Rightarrow a x=x a$, ou seja, $a$ pertence ao centro de $F$. Mas sendo $F$ um grupo livre de rank maior que 1, seu centro é trivial. Logo $a=1$ e como $a$ é arbitrário, segue que $\mathcal{I}$ é injetora.

Lema 1.6.2. Suponhamos que a sequência de grupos e homomorfismos

$$
1 \longrightarrow \mathbb{Z} \stackrel{f}{\longrightarrow} \mathbb{Z} \stackrel{g}{\longrightarrow} G \stackrel{h}{\longrightarrow} H \longrightarrow 1
$$

seja exata. Suponhamos ainda que $f: \mathbb{Z} \rightarrow \mathbb{Z}$ seja a multiplicação por $n \neq 0$. Então, o homomorfismo $\bar{g}: \mathbb{Z} /|n| \mathbb{Z} \rightarrow G$, dado por $\bar{g}([x])=g(x)$, é bem definido e a sequência

$$
1 \longrightarrow \mathbb{Z} /|n| \mathbb{Z} \stackrel{\bar{g}}{\longrightarrow} G \stackrel{h}{\longrightarrow} H \longrightarrow 1
$$

é exata.

Prova: Vamos mostrar a boa definição de $\bar{g}$. Se $[x]=\left[x^{\prime}\right]$, então $x-x^{\prime}=k n$. Então, $g(x) \cdot\left[g\left(x^{\prime}\right)\right]^{-1}=g\left(x-x^{\prime}\right)=g(k n)=g(f(k))$. Como a imagem de $f$ é igual ao núcleo de $g$, segue que $g(x) \cdot\left[g\left(x^{\prime}\right)\right]^{-1}=1 \Rightarrow \bar{g}([x])=g(x)=g\left(x^{\prime}\right)=\bar{g}\left(\left[x^{\prime}\right]\right) . \bar{g}$ é claramente homomorfismo, já que $g$ o é. É claro também que a imagem de $\bar{g}$ está contida no núcleo de $H$. Agora seja $x \in G$, tal que $h(x)=1$. Como a imagem de $g$ é igual ao núcleo de $h$, então existe $b \in \mathbb{Z}$, tal que $g(b)=x$. Logo, $\bar{g}([b])=g(b)=x$ e portanto o núcleo de $h$ está contido no núcleo na imagem de $\bar{g}$. $h$ é sobrejetora por hipótese. Ainda resta mostrar que $\bar{g}$ é injetora. Seja $[x] \in \mathbb{Z} /|n| \mathbb{Z}$ tal que $\bar{g}([x])=1 \Rightarrow g(x)=1$. Como a imagem de $f$ é igual ao núcleo de $g$, então existe $a \in \mathbb{Z}$ tal que $n a=x \Rightarrow 0=[n a]=[x]$, e portanto $\bar{g}$ é injetora. 



$\frac{-1}{2}$

\section{Grupos de Tranças de uma Superfície}

\subsection{Introdução}

Neste capítulo vamos fazer a definição formal de uma trança de uma superfície através de duas maneiras e vamos mostrar que estas definições são de certo modo equivalentes. Na mesma linha de raciocínio, vamos definir os grupos de tranças de uma superfície.

Em todo este capítulo $M$ denotará um superfície topológica conexa sem bordo.

\subsection{Grupos de Tranças Geométrico}

Nesta seção vamos formalizar a ideia intuitiva de uma trança; como duas tranças podem ser classificadas "como sendo a mesma trança" e construir o $n$-ésimo Grupo de Tranças de uma superfície.

Sejam $n$ um inteiro positivo e $\left\{P_{1}, \ldots, P_{n}\right\}$ um conjunto de pontos dois a dois distintos de $M$.

Definição 2.2.1. Uma n-trança sobre $M$ baseada em $\left\{P_{1}, \ldots, P_{n}\right\}$ é uma $n$-upla $b=$ $\left(b_{1}, \ldots, b_{n}\right)$ de caminhos $b_{i}:[0,1] \rightarrow M \times[0,1]$, que cumprem as seguintes condições: (1) Para todo $i \in\{1, \ldots, n\}, b_{i}(0)=\left(P_{i}, 0\right)$ e $b_{i}(1)=\left(P_{j}, 1\right)$, para algum $j \in\{1, \ldots, n\}$; 
(2) Para todo $t \in[0,1]$, o conjunto $\left\{b_{1}(t), \ldots, b_{n}(t)\right\}$ tem $n$ pontos distintos.

Cada caminho $b_{i}$ é chamado a i-ésima corda da trança, ou simplesmente, uma corda da trança.

O conjunto de todas as $n$-tranças sobre $M$ baseadas em $\left\{P_{1}, \ldots, P_{n}\right\}$ será denotado por $\mathcal{B}_{n}(M)$.

Quando não houver perigo de confusão, diremos que um elemento de $\mathcal{B}_{n}(M)$ é simplesmente uma trança.

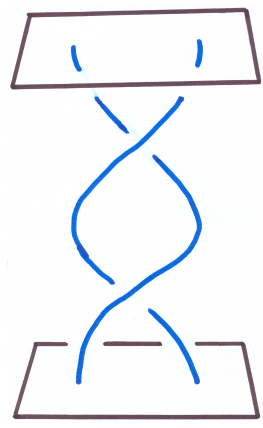

Figura 2.1: 2-trança do

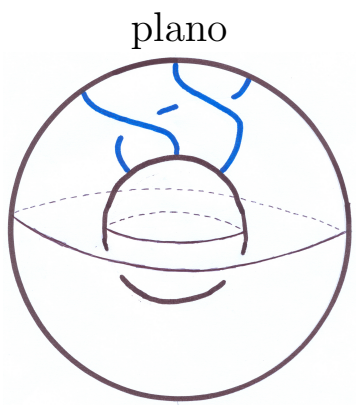

Figura 2.2: 3-trança da

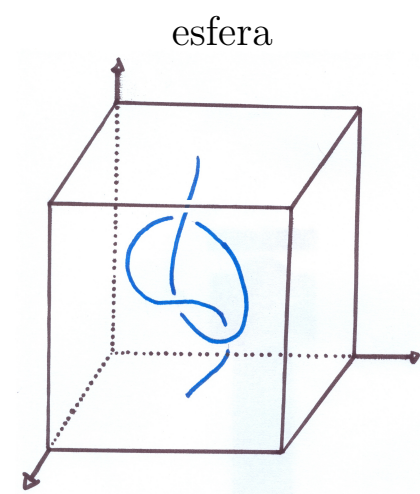

Figura 2.3: não é um trança
As figuras 2.1 e 2.2 mostram uma 2-trança do plano e uma 3-trança da esfera, respectivamente. Na verdade, estas figuras representam a imagem de 2 caminhos em $\mathbb{R}^{2} \times[0,1]$ e de 3 caminhos em $\mathbb{S}^{2} \times[0,1]$ que cumprem a definição de trança. Chamaremos a imagem dos caminhos que compõem uma trança de esquema da trança. A figura 2.3 mostra um caminho em $Q \times[0,1]$ que não cumpre a condição (2) da definição de trança, sendo $Q$ o quadrado unitário aberto do plano.

Dada uma trança $b=\left(b_{1}, \ldots, b_{n}\right) \in \mathcal{B}_{n}(M)$, podemos associar uma permutação $\bar{\alpha}(b) \in S_{n}$, chamada permutação da trança $b$, da seguinte maneira:

Para cada $i \in\{1, \ldots, n\}$, seja $j \in\{1, \ldots, n\}$ tal que $b_{i}(1)=\left(P_{j}, 1\right)$. Definimos $\bar{\alpha}(b)(i)=j$. De fato $\bar{\alpha}(b)$ é uma permutação pela condição (2) da definição de trança.

Se $\bar{\alpha}(b)$ é a permutação trivial, então $b$ é dita uma $n$ trança pura. A trança da figura 2.1 é uma 2-trança pura, enquanto a trança da figura 2.2 não é pura.

Consideremos $\Pi_{M}: M \times[0,1] \rightarrow M$ a projeção no primeiro fator. Dada uma $n$-trança $b=\left(b_{1}, \ldots, b_{n}\right)$, denotaremos por $\Pi_{M}(b):=\left(\Pi_{M} \circ b_{1}, \ldots, \Pi_{M} \circ b_{n}\right)$ a $n$-upla de caminhos em $M$.

Dadas duas tranças $a=\left(a_{1}, \ldots, a_{n}\right)$ e $b=\left(b_{1}, \ldots, b_{n}\right)$ em $\mathcal{B}_{n}(M)$, vamos associar uma terceira trança $a b$, chamada produto da trança $a$ com a trança $b$.

Para cada $i \in\{1, \ldots, n\}$, seja $(a b)_{i}:[0,1] \rightarrow$ $M \times[0,1]$, dada por $(a b)_{i}(t)=\left(\left(\Pi_{M} \circ a_{i}\right) *\left(\Pi_{M} \circ\right.\right.$ $\left.\left.b_{\bar{\alpha}(a)(i)}\right)(t), t\right)$, sendo que $*$ denota a justaposição de caminhos e $\bar{\alpha}(a)$ a permutação da trança $a$. Definimos $a b=\left((a b)_{1}, \ldots,(a b)_{n}\right)$. 
A figura 2.4 dá uma ideia geométrica do produto de tranças.

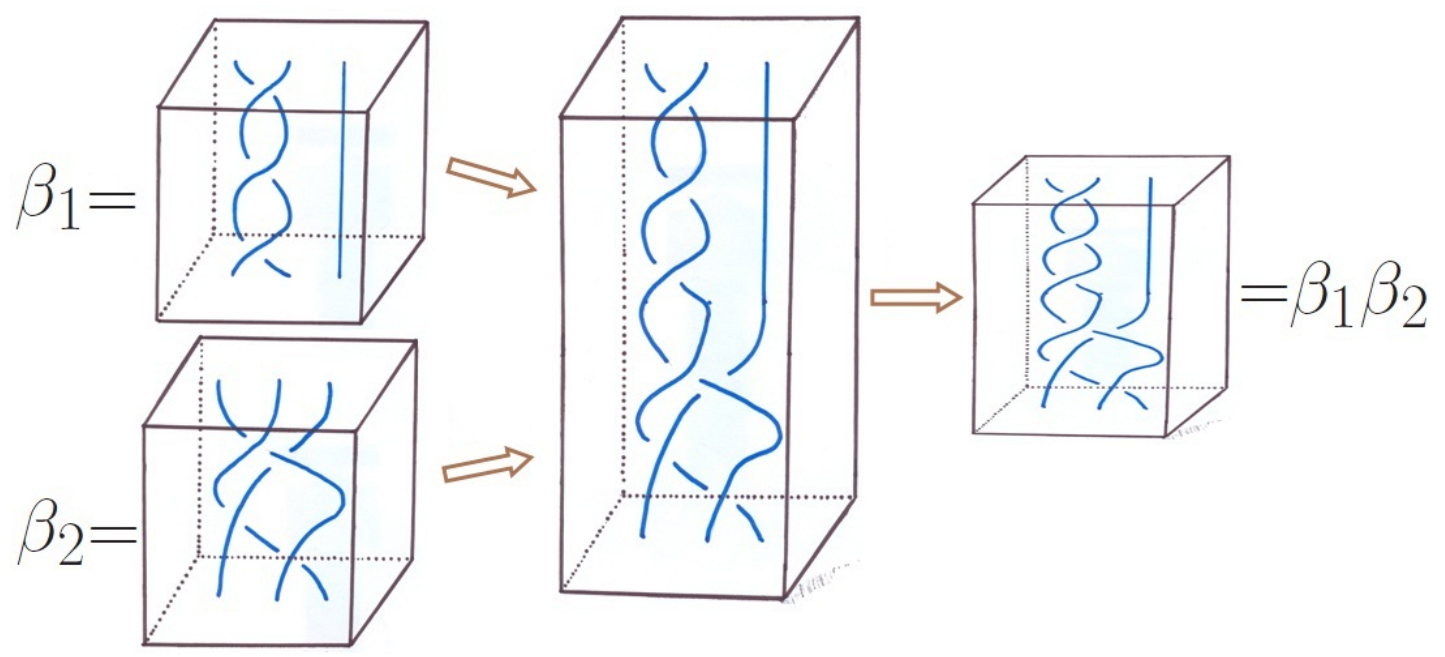

Figura 2.4: produto de tranças

Este produto de tranças não fornece uma estrutura de grupo a $\mathcal{B}_{n}(M)$, pois não existe um elemento neutro bem definido. Entretanto, existe uma relação de equivalência em $\mathcal{B}_{n}(M)$, a qual definimos abaixo, que torna o conjunto das classes um grupo com a operação induzida.

Definição 2.2.2. Dadas duas tranças $a=\left(a_{1}, \ldots, a_{n}\right)$ e $b=\left(b_{1}, \ldots, b_{n}\right)$ em $\mathcal{B}_{n}(M)$ com mesma permutação, dizemos que a é equivalente a $b$, e denotamos $a \cong b$, se existem aplicações contínuas $F_{i}:[0,1] \times[0,1] \rightarrow M \times[0,1], i=1, \ldots, n$, tais que:

(i) Para todo $s \in[0,1]$, se definirmos $f_{i}^{s}:[0,1] \rightarrow M \times[0,1]$ por $f_{i}^{s}(t)=F_{i}(t, s)$, então $F^{s}=\left(f_{1}^{s}, \ldots, f_{n}^{s}\right)$ é uma $n$-trança baseada em $\left\{P_{1}, \ldots, P_{n}\right\}$, com mesma permutação de a (e consequentemente de $b)$;

(ii) $F^{0}=$ a e $F^{1}=b$.

Intuitivamente, duas tranças são equivalentes, se podemos deformar continuamente as cordas de uma nas cordas da outra sem que cordas distintas se toquem e durante a deformação os extremos ficam fixos. A figura 2.5 mostra duas 3-tranças do plano que não são equivalentes, pois não tem mesma permutação. A figura 2.6 mostra duas 2-tranças do plano em que não é possível deformar continuamente as cordas de uma nas cordas da outra. Ao longo desta dissertação veremos muitas tranças equivalentes.

Teorema 2.2.3. A relação $\cong$ é uma relação de equivalência e o conjunto $B_{n}(M):=$ $\mathcal{B}_{n}(M) / \cong$ é um grupo com o produto induzido, chamado o grupo de $n$-tranças de $M$ ou grupo de tranças com $n$ cordas de $M$. 

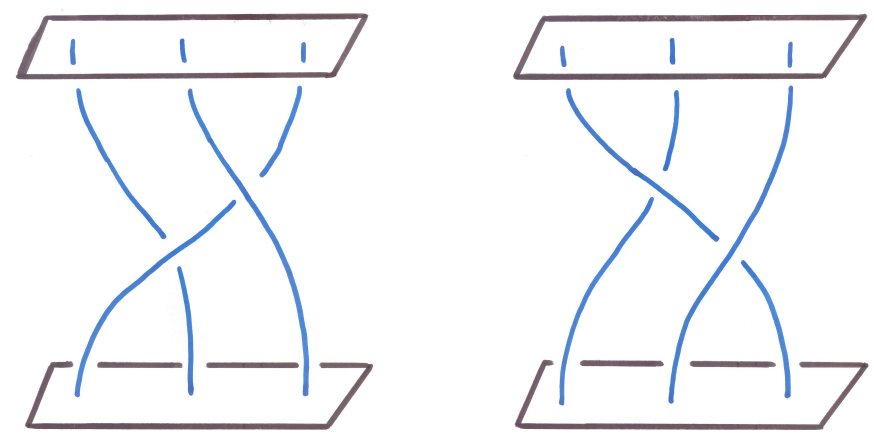

Figura 2.5: tranças que não tem mesma permutação
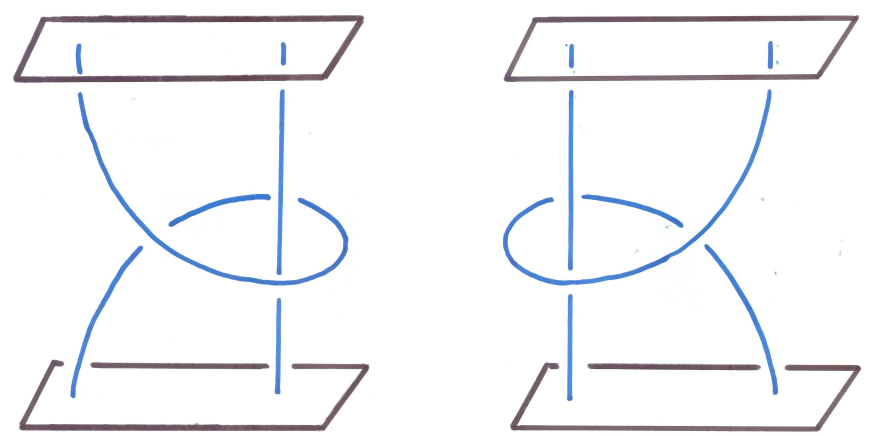

Figura 2.6: tranças com mesma permutação, mas que não são equivalentes

Prova: A demonstração é totalmente análoga a que mostra que o conjunto das classes de homotopia dos laços com ponto base fixado de um espaço topológico com a operação justaposição de caminhos é um grupo (Grupo Fundamental) e citamos [13] como referência.

Observação 2.2.4. A trança $1=\left(k_{1}, \ldots, k_{n}\right)$, sendo $k_{i}(t)=\left(P_{i}, t\right)$ para todo $t \in[0,1]$, representa a classe de equivalência do elemento neutro de $B_{n}(M)$. Se $b=\left(b_{1}, \ldots, b_{n}\right)$ é uma $n$-trança, então a trança $b^{-1}=\left(b_{1}^{-1}, \ldots, b_{n}^{-1}\right)$, sendo $b_{i}^{-1}:[0,1] \rightarrow M \times[0,1]$ o caminho definido por $b_{i}^{-1}(t)=\left(\Pi_{M} \circ b_{\bar{\alpha}(b)^{-1}(i)}(1-t), t\right)$, é tal que $\left[b^{-1}\right]=[b]^{-1}$. As tranças representadas nas figuras 2.5 e 2.6 são tais que suas classes de equivalência são inversa uma da outra.

Notemos que se $a \cong b$, então a permutação de $a$ é igual a permutação de $b$. Logo, a função $\alpha: B_{n}(M) \rightarrow S_{n}$ dada por $\alpha([b])=\bar{\alpha}(b)$ está bem definida. Pela definição de produto de tranças, segue que $\alpha$ é um homomorfismo.

Definição 2.2.5. O núcleo do homomorfismo a é chamado grupo de n-tranças puras de $M$ ou grupo de tranças puras com $n$ cordas de $M$ e denotado por $P_{n}(M)$.

Teorema 2.2.6. $B_{n}(M) / P_{n}(M)$ é isomorfo a $S_{n}$. 
Prova: Pelo teorema dos isomorfismos, basta mostrar que $\alpha$ é sobrejetora. E para mostrar que $\alpha$ é sobrejetora, basta mostrar que se $(i, i+1) \in S_{n}$ é uma transposição, existe uma trança $a$, tal que $\bar{\alpha}(a)=(i, i+1)$, pois as transposições geram $S_{n}$. Sendo $M$ superfície topológica conexa sem bordo, então existem caminhos $A_{i}, A_{i+1}:[0,1] \rightarrow M$ tais que $A_{i}(0)=A_{i+1}(1)=P_{i}, A_{i}(1)=A_{i+1}(0)=P_{i+1}, A_{i}(t) \neq A_{i+1}(t)$ para todo $t \in[0,1]$ e $A_{i}(t), A_{i+1}(t) \neq P_{j}$ para todo $j \neq i, i+1$. A trança $a=\left(a_{1}, \ldots, a_{n}\right)$, sendo $a_{j}:[0,1] \rightarrow M \times[0,1]$ definidos por

$$
a_{j}(t)=\left\{\begin{array}{l}
\left(A_{i}(t), t\right), \text { se } j=i \\
\left(A_{i+1}(t), t\right), \text { se } j=i+1 \\
\left(P_{j}, t\right), \text { caso contrário }
\end{array}\right.
$$

cumpre $\bar{\alpha}(a)=(i, i+1)$

Notemos que na definição do grupo de $n$-tranças de $M$, não fizemos menção ao conjunto de pontos $\left\{P_{1}, \ldots, P_{n}\right\}$, pois a escolha destes pontos não altera a estrutura algébrica deste grupo. Mais precisamente: se $\left\{P_{1}^{\prime}, \ldots, P_{n}^{\prime}\right\}$ é outro conjunto de $n$ pontos distintos de $M$ e $B_{n}^{\prime}(M)$ é o grupo de $n$-tranças de $M$, mas agora construído a partir destes novos pontos, então $B_{n}(M)$ e $B_{n}^{\prime}(M)$ são isomorfos. Este fato será mostrado na próxima seção.

\subsection{Grupos de Tranças e Espaços de Configuração}

Nesta seção vamos dar uma nova definição dos Grupos de Tranças de uma superfície através da noção de Espaços de Configuração. O caminho aqui é de certo modo, reverso do tópico anterior, pois aqui primeiro definimos os Grupos de Tranças e depois uma trança.

Definição 2.3.1. Seja $\left[x^{0}\right]=\left[\left(x_{1}^{0}, \ldots, x_{n}^{0}\right)\right]$ um ponto fixado de $C_{n}(M)$. O grupo $\pi_{1}\left(C_{n}(M),\left[x^{0}\right]\right)$ é chamado grupo das $n$-tranças de $M$ ou grupo de tranças com $n$ cordas de $M$. Quando $n$ estiver subtendido, chamaremos este grupo simplesmente de grupo de tranças de $M$.

O grupo de tranças de $M$ não depende do ponto base escolhido. De fato, como observado na seção "Espaços de Configuração", o espaço $F_{n}(M)$ é conexo e sendo variedade, então é conexo por caminhos. Sendo a projeção $p: F_{n}(M) \rightarrow C_{n}(M)$ um recobrimento (corolário 1.2.6), portanto contínua e sobrejetora, então $C_{n}(M)$ é conexo por caminhos. 
Se $[b] \in \pi_{1}\left(C_{n}(M),\left[x^{0}\right]\right)$, então o caminho $b:[0,1] \rightarrow C_{n}(M)$ é dito uma trança com $n$ cordas de $M$.

Precisamos mostrar que esta nova definição de trança e de grupo de tranças "coincide" com a anterior e isto é dado pelo

Teorema 2.3.2. Seja $\left[x^{0}\right]=\left[\left(x_{1}^{0}, \ldots, x_{n}^{0}\right)\right] \in C_{n}(M)$. Então os grupos $\pi_{1}\left(C_{n}(M),\left[x^{0}\right]\right)$ e $B_{n}(M)$ são isomorfos, sendo $B_{n}(M)$ o grupo de $n$-tranças de $M$, construído com base nos pontos $\left\{x_{1}^{0}, \ldots, x_{n}^{0}\right\}$.

Prova: A prova será construir homomorfismos $\lambda: \pi_{1}\left(C_{n}(M),\left[x^{0}\right]\right) \rightarrow B_{n}(M)$ e $\mu$ : $B_{n}(M) \rightarrow \pi_{1}\left(C_{n}(M),\left[x^{0}\right]\right)$ tais que $\mu \circ \lambda=I d_{\pi_{1}\left(C_{n}(M),\left[x^{0}\right]\right)}$ e $\lambda \circ \mu=I d_{B_{n}(M)}$.

Sejam $\beta \in \pi_{1}\left(C_{n}(M),\left[x^{0}\right]\right)$ e $b:[0,1] \rightarrow C_{n}(M)$ um caminho fechado tal que $[b]=\beta$. Sendo a projeção natural $p: F_{n}(M) \rightarrow C_{n}(M)$ um recobrimento, existe um único levantamento de $b$ pelo ponto $x^{0}=\left(x_{1}^{0}, \ldots, x_{n}^{0}\right)$, digamos $\bar{b}:[0,1] \rightarrow F_{n}(M)$. Notemos que $\bar{b}(t)=\left(b_{1}(t), \ldots, b_{n}(t)\right)$, sendo $b_{i}$ caminhos em $M$ tais que se $i \neq j$, então $b_{i}(t) \neq b_{j}(t)$, para todo $t \in[0,1]$. Notemos ainda que sendo $b$ um caminho fechado, então existe uma única permutação $s \in S_{n}$, tal que $b_{i}(1)=x_{s(i)}^{0}$. Logo, definindo $a_{i}:[0,1] \rightarrow M \times[0,1]$ por $a_{i}(t)=\left(b_{i}(t), t\right)$, temos que $a=\left(a_{1}, \ldots, a_{n}\right)$ é uma $n$-trança sobre $M$ baseada em $\left\{x_{1}^{0}, \ldots x_{n}^{0}\right\}$. Definimos $\lambda(\beta)=[a]$.

Precisamos mostrar que $\lambda$ está bem definida, no sentido que se $b^{\prime}$ é outro laço em $C_{n}(M)$ que representa a classe $\beta$, então a trança construída a partir de $b^{\prime}$, digamos $a^{\prime}$, é tal que $[a]=\left[a^{\prime}\right]$.

Seja $\overline{b^{\top}}$ o único levantamento de $b^{\prime}$ a partir de $x^{0}$. Novamente, notemos que $\overline{b^{\top}}(t)=$ $\left(\overline{b_{1}}(t), \ldots, \overline{b_{n}}(t)\right)$, sendo $\overline{b_{i}{ }^{\top}}$ caminhos em $M$ tais que se $i \neq j$, então $\overline{b_{i}}(t) \neq \overline{b_{j}{ }^{\top}}(t)$, para todo $t \in[0,1]$. A trança construída a partir de $\overline{b^{\top}}$ é $a^{\prime}=\left(a_{1}^{\prime}, \ldots, a_{n}^{\prime}\right)$, sendo $a_{i}^{\prime}(t)=\left(\overline{b_{i}}(t), t\right)$.

Como $p$ é recobrimento e $\beta=[b]=\left[b^{\prime}\right]$, então $\overline{b_{i}^{\prime}}(1)=x_{s(i)}^{0}=b_{i}^{\prime}(1)$ e existe uma homotopia $F:[0,1] \times[0,1] \rightarrow F_{n}(M)$, tal que $F(t, 0)=\bar{b}(t), F(t, 1)=\bar{b}(t)$, $F(0, s)=\left(x_{1}^{0}, \ldots, x_{n}^{0}\right)$ e $F(1, s)=\left(x_{s(1)}^{0}, \ldots, x_{s(n)}^{0}\right)$. Logo, se denotarmos $F(t, s)=$ $\left(f_{1}(t, s), \ldots, f_{n}(t, s)\right)$, então estão bem definida as funções $F_{i}:[0,1] \times[0,1] \rightarrow M \times[0,1]$, dadas por $F_{i}(t, s)=\left(f_{i}(t, s), t\right)$. Notemos que as funções $F_{i}$ 's cumprem as condições (i) e (ii) da definição 2.2.2 para as tranças $a$ e $a^{\prime}$. Logo, $[a]=\left[a^{\prime}\right]$ e a função $\lambda$ está bem definida.

Agora vamos mostrar que $\lambda$ é homomorfismo. Sejam $[c]$ e $[d]$ pertencentes a $\pi_{1}\left(C_{n}(M), x^{0}\right)$ e $\bar{c}$ e $\bar{d}$ os levantamentos a partir de $x^{0}$ dos laços $c$ e $d$, respectivamente. Escrevendo $\bar{c}(t)=\left(c_{1}(t), \ldots, c_{n}(t)\right)$ e $\bar{d}(t)=\left(d_{1}(t), \ldots, d_{n}(t)\right)$, então as tranças construídas a partir de $\bar{c}$ e $\bar{d}$ são, respectivamente, $T^{c}=\left(T_{1}^{c}, \ldots, T_{n}^{c}\right)$ e $T^{d}=\left(T_{1}^{d}, \ldots, T_{n}^{d}\right)$, sendo $T_{i}^{c}:[0,1] \rightarrow M \times[0,1]$ definida por $T_{i}^{c}(t)=\left(c_{i}(t), t\right)$ e $T_{i}^{d}:[0,1] \rightarrow M \times[0,1]$ 
definida por $T_{i}^{d}(t)=\left(d_{i}(t), t\right), i=1, \ldots, n$. Logo, por definição

$$
\lambda([c])=\left[T^{c}\right] \text { e } \lambda([d])=\left[T^{d}\right] .
$$

Se $s$ é a permutação da trança $T^{c}$, então a i-ésima corda da trança $T^{c} T^{d}$ é dada pelo caminho definido por $T_{i}^{c} T_{s(i)}^{d}(t)=\left(c_{i} * d_{s(i)}(t), t\right)$. Agora notemos que o caminho definido por $\left(c_{1} * d_{s(1)}(t), \ldots, c_{n} * d_{s(n)}(t)\right)$ é um levantamento do laço $c * d$ e a trança construída a partir deste caminho é justamente $T^{c} T^{d}$. Logo

$$
\lambda([c][d])=\lambda([c * d])=\left[T^{c} T^{d}\right]=\left[T^{c}\right]\left[T^{d}\right]=\lambda([c]) \lambda([d])
$$

e portanto $\lambda$ é homomorfismo.

O homomorfismo $\mu$ é construído da seguinte maneira: se $[a] \in B_{n}(M)$ é uma $n$ trança baseada em $\left\{x_{1}^{0}, \ldots x_{n}^{0}\right\}$, então $a=\left(a_{1}, \ldots, a_{n}\right)$, sendo $a_{i}$ caminhos em $M \times[0,1]$, $a_{i}(0)=\left(x_{i}^{0}, 0\right)$ e $a_{i}(1)=\left(x_{s(i)}^{0}, 1\right)$, para algum $s \in S_{n}$. Sendo $\Pi_{M}: M \times[0,1] \rightarrow M$ a projeção no primeiro fator, temos que $\Pi_{M}(a):[0,1] \rightarrow F_{n}(M)$ dado por $\Pi_{M}(a)(t)=$ $\left(\Pi_{M} \circ a_{1}(t), \ldots, \Pi_{M} \circ a_{n}(t)\right)$ é um caminho com ponto inicial $x^{0}=\left(x_{1}^{0}, \ldots x_{n}^{0}\right)$, bem definido por causa da condição (2) da definição de trança. Notemos que $p \circ \Pi_{M}(a)$ : $[0,1] \rightarrow C_{n}(M)$ é um laço com ponto base $\left[x^{0}\right]=\left[\left(x_{1}^{0}, \ldots, x_{n}^{0}\right)\right]$.

Definimos $\mu([a])=\left[p \circ \Pi_{M}(a)\right]$. Através de argumentos totalmente análogos aos anteriores mostra-se que $\mu$ está bem definida, é um homomorfismo de grupos e é fácil ver que realmente $\lambda$ e $\mu$ são inversas uma da outra.

Corolário 2.3.3. Se $B_{n}(M)$ e $B_{n}^{\prime}(M)$ são grupos de $n$-tranças de $M$ construídos a partir de $\left\{x_{1}^{0}, \ldots, x_{n}^{0}\right\}$ e $\left\{x_{1}^{1}, \ldots, x_{n}^{1}\right\}$, respectivamente, então $B_{n}(M)$ e $B_{n}^{\backslash}(M)$ são isomorfos.

Prova: Colocando $x^{0}=\left(x_{1}^{0}, \ldots, x_{n}^{0}\right)$ e $x^{1}=\left(x_{1}^{1}, \ldots, x_{n}^{1}\right)$, temos pelo teorema que $\pi_{1}\left(C_{n}(M),\left[x^{0}\right]\right)$ e $\pi_{1}\left(C_{n}(M),\left[x^{1}\right]\right)$ são isomorfos a $B_{n}(M)$ e $B_{n}^{\backslash}(M)$, respectivamente. Mas $C_{n}(M)$ é conexo por caminhos, $\operatorname{logo} \pi_{1}\left(C_{n}(M),\left[x^{0}\right]\right)$ e $\pi_{1}\left(C_{n}(M),\left[x^{1}\right]\right)$ são isomorfos. Segue o resultado.

Dado uma trança $\beta \in \pi_{1}\left(C_{n}(M),\left[x^{0}\right]\right), \beta=[b]$, podemos associar uma permutação $s(\beta)$, da seguinte maneira: $s(\beta)=\alpha \circ \lambda([b])$, sendo $\alpha$ o homomorfismo definido na seção anterior e $\lambda$ o homomorfismo definido na demonstração do teorema 2.3.2. Segue que a função $s: \pi_{1}\left(C_{n}(M),\left[x^{0}\right]\right) \rightarrow S_{n}$, dada por $s(\beta)=\alpha \circ \lambda(\beta)$ está bem definida e é um homomorfismo.

Como na seção anterior, temos a seguinte: 
Definição 2.3.4. O núcleo do homomorfismo s é chamado de grupo de $n$-tranças puras de $M$ ou grupo de tranças puras de $M$ com $n$ cordas.

Observação 2.3.5. Notemos que o grupo de $n$-tranças puras de $M$, no sentindo da definição acima, é isomorfo a $P_{n}(M)$, por causa da definição de $s$ e pelo fato de $\lambda$ ser isomorfismo. Mais ainda, temos o seguinte resultado:

Teorema 2.3.6. O grupo de $n$-tranças puras de $M$ é isomorfo a $\pi_{1}\left(F_{n}(M), x^{0}\right)$.

Prova: Pela observação acima, basta construir homomorfismos $\gamma: \pi_{1}\left(F_{n}(M), x^{0}\right) \rightarrow$ $P_{n}(M)$ e $\eta: P_{n}(M) \rightarrow \pi_{1}\left(F_{n}(M), x^{0}\right)$, tais que $\gamma \circ \eta=I d_{P_{n}(M)}$ e $\eta \circ \gamma=I d_{\pi_{1}\left(F_{n}(M), x^{0}\right)}$. Seja $\alpha \in \pi_{1}\left(F_{n}(M), x^{0}\right), \alpha=[a] . a(t)=\left(a_{1}(t), \ldots, a_{n}(t)\right)$, sendo $a_{i}:[0,1] \rightarrow M$ laços com ponto base $x_{i}^{0}$ e tais que se $i \neq j$, então $a_{i}(t) \neq a_{j}(t)$. Logo $\bar{a}=\left(\overline{a_{1}}(t), \ldots, \overline{a_{n}}(t)\right)$, sendo $\overline{a_{i}} \rightarrow M \times[0,1]$ dadas por $\overline{a_{i}}(t)=\left(a_{i}(t), t\right)$, é uma $n$-trança pura. Definimos $\gamma(\alpha)=\bar{a}$. A demonstração que $\gamma$ está bem definida e é um homomorfismo é totalmente análoga a que a função $\lambda$ do teorema 2.3.2 está bem definida e é um homomorfismo. Agora se $[b] \in P_{n}(M)$, então $b=\left(b_{1}, \ldots, b_{n}\right)$, sendo $b_{i}:[0,1] \rightarrow M \times[0,1]$ caminhos tais que $b_{i}(0)=\left(x_{i}^{0}, 0\right), b_{i}(1)=\left(x_{i}^{0}, 1\right)$ e se $i \neq j$, então $b_{i}(t) \neq b_{j}(t)$, para todo $t \in[0,1]$. Definimos $\eta([b])=\left[\Pi_{M} \circ b\right]$, sendo $\Pi_{M} \circ b:[0,1] \rightarrow F_{n}(M)$ dada por $\Pi_{M} \circ b(t)=\left(\Pi_{M} \circ b_{1}(t), \ldots, \Pi_{M} \circ b_{n}(t)\right)$. Novamente, a demonstração que $\eta$ está bem definida e é um homomorfismo são análogas a que $\mu$ do teorema 2.3.2 está bem definida. E é fácil ver $\gamma$ e $\eta$ são inversas uma da outra.

Corolário 2.3.7. Se $[b] \in \pi_{1}\left(C_{n}(M),\left[x^{0}\right]\right)$ é tal que $s([b])$ é a permutação trivial, então o levantamento de $b$ (pelo recobrimento $p: F_{n}(M) \rightarrow C_{n}(M)$ ) é um laço com ponto base $x^{0}$.

Prova: Segue diretamente da construção dos isomorfismos $\lambda$ e $\gamma$ dos teoremas 2.3 .2 e 2.3.6.

Corolário 2.3.8. $\pi_{1}\left(C_{n}(M),\left[x^{0}\right]\right) / p_{*}\left(\pi_{1}\left(F_{n}(M), x^{0}\right)\right)$ é isomorfo a $S_{n}$, sendo $p_{*}$ a induzida nos grupo fundamentais do recobrimento $p: F_{n}(M) \rightarrow C_{n}(M)$.

Prova: Sendo $p$ recobrimento, então $\pi_{1}\left(F_{n}(M), x^{0}\right)$ é isomorfo a $p_{*}\left(\left(\pi_{1}\left(F_{n}(M), x^{0}\right)\right)\right.$. Pelo teorema 2.3.6, $\pi_{1}\left(F_{n}(M), x^{0}\right)$ é isomorfo a $P_{n}(M)$. Como $\pi_{1}\left(C_{n}(M),\left[x^{0}\right]\right)$ é isomorfo a $B_{n}(M)$, segue do teorema 2.2 .6 que $\pi_{1}\left(C_{n}(M),\left[x^{0}\right]\right) / p_{*}\left(\pi_{1}\left(F_{n}(M), x^{0}\right)\right)$ é isomorfo a $S_{n}$.

Observação 2.3.9. O grupo de 1-tranças de $M$ é igual ao $\pi_{1}(M)$. Isto decorre diretamente do fato de $F_{1}(M)=M$. 
Em todo restante desta dissertação não faremos distinção entre os grupos $\pi_{1}\left(C_{n}(M),\left[x^{0}\right]\right)$ e $B_{n}(M)$, que serão chamados de grupos de $n$-tranças de $M$ ou grupo de tranças com $n$ cordas de $M$. Da mesma maneira, não faremos distinção entre os grupos $\pi_{1}\left(F_{n}(M), x^{0}\right)$ e $P_{n}(M)$, que serão chamados de grupos de $n$-tranças puras de $M$ ou grupo de tranças com $n$ cordas de $M$.

Um elemento que representa a classe de equivalência de alguma trança, também será chamado trança, quando não houver perigo de confusão. Se $\beta$ é uma trança, independente se estivermos olhando como elemento de $\pi_{1}\left(C_{n}(M),\left[x^{0}\right]\right)$ ou $B_{n}(M)$, então a permutação de $\beta$ será denotada por $\alpha(\beta)$ em todo restante desta dissertação.

A vantagem de se olhar uma trança como elemento de $B_{n}(M)$ é a intuição geométrica a qual não se tem muito em $\pi_{1}\left(C_{n}(M),\left[x^{0}\right]\right)$. Entretanto, olhando uma trança como elemento deste último grupo, temos todas as boas propriedades da teoria de grupo fundamental e recobrimento a disposição. 



$\frac{-1}{3}$

\section{Grupos de Tranças do Plano}

\subsection{Introdução}

No capítulo anterior fizemos um estudo dos grupos de tranças de uma superfície topológica conexa e sem bordo. Neste capítulo, vamos particularizar esta discussão para o caso clássico $\mathbb{R}^{2}$, que é de grande importância, pelo fato que $B_{n}\left(\mathbb{R}^{2}\right)$ pode ser considerado de maneira natural como subgrupo de $B_{n}(M)$ para uma quantidade grande de superfícies. Este fato será formalizado na última seção.

Apresentações de $B_{n}\left(\mathbb{R}^{2}\right)$ e $P_{n}\left(\mathbb{R}^{2}\right)$ serão dadas nas seções 1 e 2 , respectivamente. Não faremos a demonstração destas apresentações, apenas algumas justificativas geométricas. Estas demonstrações estão feitas em detalhes em [6], [7] e [8].

\subsection{Apresentação de $B_{n}\left(\mathbb{R}^{2}\right)$}

Seja $n \geq 2$ e consideremos o conjunto de pontos $\{(1,0), \ldots,(n, 0)\} \subset \mathbb{R}^{2}$. Fixemos $i \in\{1, \ldots, n-1\}$ e consideremos os caminhos $a_{i}, a_{i+1}:[0,1] \rightarrow \mathbb{R}^{2}$ definidos por:

$$
a_{i}(t)=(i+t,-\operatorname{sen}(\pi t)) \text { e } a_{i+1}(t)=(i+1-t, \operatorname{sen}(\pi t))
$$

Na figura 3.1 as linhas vermelha e verde representam, respectivamente, as imagens dos caminhos $a_{i}$ e $a_{i+1}$. 


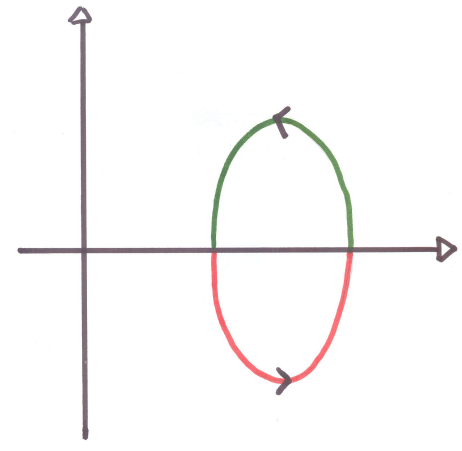

Figura 3.1: imagem dos caminhos $a_{i}$ e $a_{i+1}$

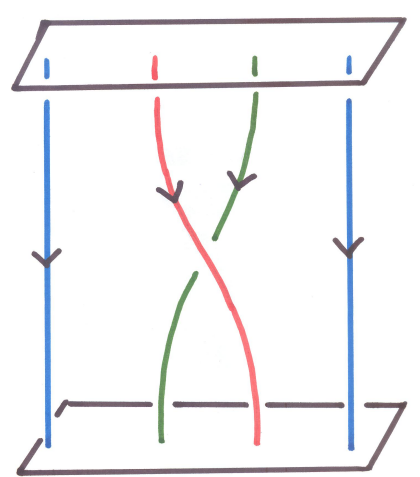

Figura 3.2: trança $\sigma_{i}$

Agora, para cada $j=1, \ldots, n$, seja $b_{j}:[0,1] \rightarrow \mathbb{R}^{2} \times[0,1]$ o caminho definido por:

$$
b_{j}(t)=\left\{\begin{array}{l}
\left(a_{i}(t), t\right), \text { se } j=i \\
\left(a_{i+1}(t), t\right), \text { se } j=i+1 \\
((j, 0), t), \text { se } j \neq i, i+1
\end{array}\right.
$$

A $i$-ésima trança de Artin, denotada por $\sigma_{i}$, é a trança $\left(b_{i}, \ldots, b_{n}\right)$.

Na figura 3.2, temos um esquema da trança $\sigma_{i}$, sendo que as linhas vermelha e verde representam, respectivamente, a $i$-ésima e a $(i+1)$-ésima corda.

A importância das tranças de Artin é que elas geram $B_{n}\left(\mathbb{R}^{2}\right)$.
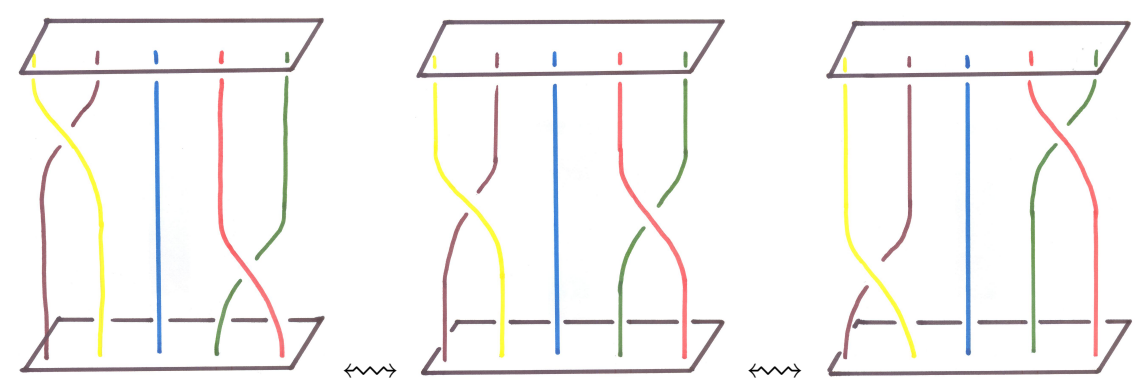

Figura 3.3: relação (i)
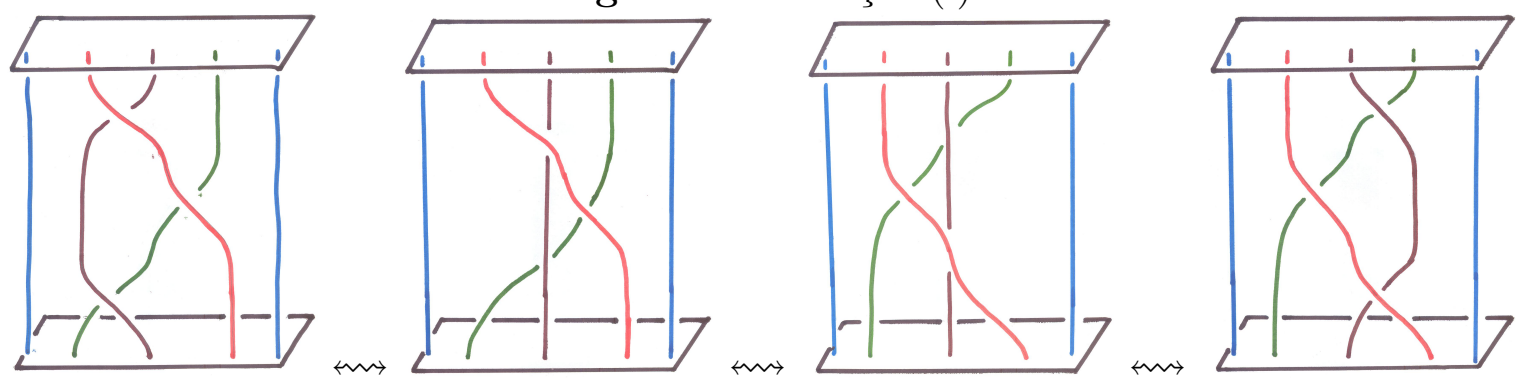

Figura 3.4: relação (ii) 
Os esquemas das figuras 3.3 e 3.4 mostram que as tranças de Artin estão sujeitas as seguintes relações:

(i) $\sigma_{i} \sigma_{j}=\sigma_{j} \sigma_{i}$, sempre que $|i-j| \geq 2$;

(ii) $\sigma_{i} \sigma_{i+1} \sigma_{i}=\sigma_{i+1} \sigma_{i} \sigma_{i+1}$.

Estas relações são chamadas de relações de Artin e definem um completo sistema de relações para $B_{n}\left(\mathbb{R}^{2}\right)$, isto é, todas as outras relações provêm destas.

Temos então o:

Teorema 3.2.1. $B_{n}\left(\mathbb{R}^{2}\right)$ admite a seguinte apresentação:

Geradores: $\sigma_{1}, \ldots, \sigma_{n-1}$

Relações: (i) $\sigma_{i} \sigma_{j}=\sigma_{j} \sigma_{i}$, sempre que $|i-j| \geq 2$;

(ii) $\sigma_{i} \sigma_{i+1} \sigma_{i}=\sigma_{i+1} \sigma_{i} \sigma_{i+1}$.

\subsection{Apresentação de $P_{n}\left(\mathbb{R}^{2}\right)$}

Seja $n \geq 2$. Consideremos em $P_{n}\left(\mathbb{R}^{2}\right)$, para $1 \leq i<j \leq$ $n$, as seguintes tranças:

$$
a_{i, j}:=\sigma_{i}^{-1} \ldots \sigma_{j-1}^{-1} \sigma_{j}^{2} \sigma_{j-1} \ldots \sigma_{i} .
$$

As figuras 3.5 e 3.6 mostram, respectivamente, os esquemas das tranças $a_{i, j}$ e $a_{i, j}^{-1}$, sendo que as linhas verde e vermelha representam a $j$-ésima e a $i$-ésima corda.

A importância das tranças $\left\{a_{i, j}\right\}_{1 \leq i<j \leq n}$ é que elas geram $P_{n}\left(\mathbb{R}^{2}\right)$.

Seja $b$ uma $n$-trança pura. Vamos dar uma ideia geométrica de como $b$ se escreve como produto das tranças $\left\{a_{i, j}\right\}_{1 \leq i<j \leq n}$ e suas inversas.

Troquemos a primeira corda de $b$ por um segmento vertical, sem "enlaçamentos" com as demais cordas e chamemos de $c_{1}$ a nova trança. Seja $a_{1}=b c_{1}^{-1} \Rightarrow b=a_{1} c_{1}$. Por construção, se retirarmos a primeira corda de $a_{1}$, podemos identificar as demais cordas com a $(n-1)$-trança trivial.

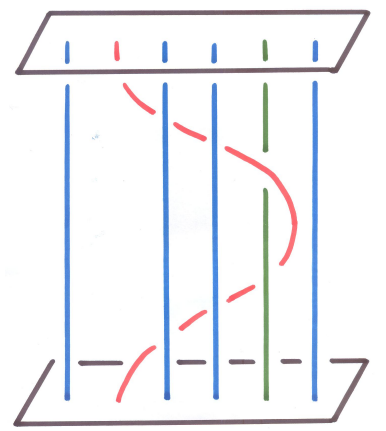

Figura 3.5: $a_{i, j}$

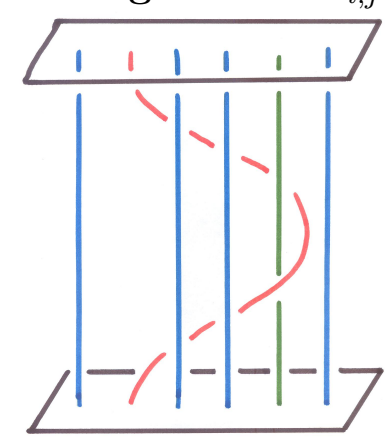

Figura 3.6: $a_{i, j}^{-1}$ Agora, troquemos a segunda corda de $c_{1}$ por um segmento vertical, sem "enlaçamentos" com as demais cordas e chamemos de $c_{2}$ a nova trança. Seja $a_{2}=c_{1} c_{2}^{-1} \Rightarrow c_{1}=$ $a_{2} c_{2} \Rightarrow b=a_{1} a_{2} c_{2}$. Por construção, se retirarmos as duas primeiras cordas de $a_{2}$, podemos identificar as demais cordas com a $(n-2)$-trança trivial. Continuando este processo, obtemos que $b=a_{1} a_{2} \ldots a_{n-1}$, sendo que cada trança pura $a_{i}$ é tal que se 


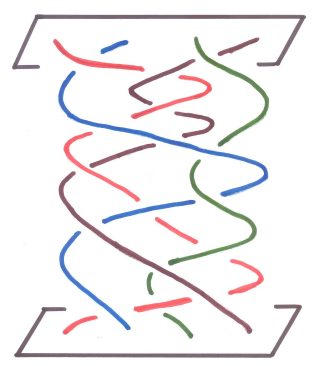

Figura 3.7: b

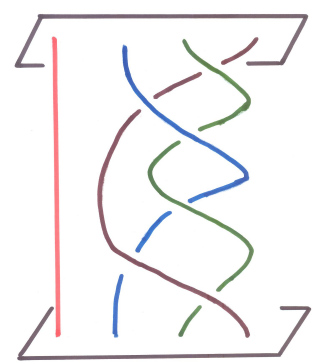

Figura 3.8: $c_{1}$

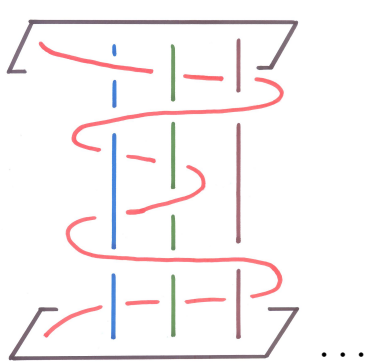

Figura 3.9: $a_{1}$

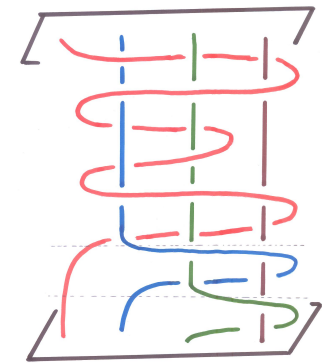

Figura 3.10: $b$

penteada

retirarmos as $i$ primeiras cordas, podemos identificar as demais cordas com a $(n-i)$ trança trivial. As tranças do tipo $a_{i}$ são chamadas tranças penteadas e a decomposição que obtivemos de $b$ é chamada um penteamento da trança.

Nas figuras 3.7, 3.8, 3.9 e 3.10 exemplificamos este processo, sendo $b$ um 4-trança.

A figura 3.11 nos dá uma ideia de como cada trança penteada pode ser escrita como produto das tranças $\left\{a_{i, j}\right\}_{1 \leq i<j \leq n}$ e suas inversas.
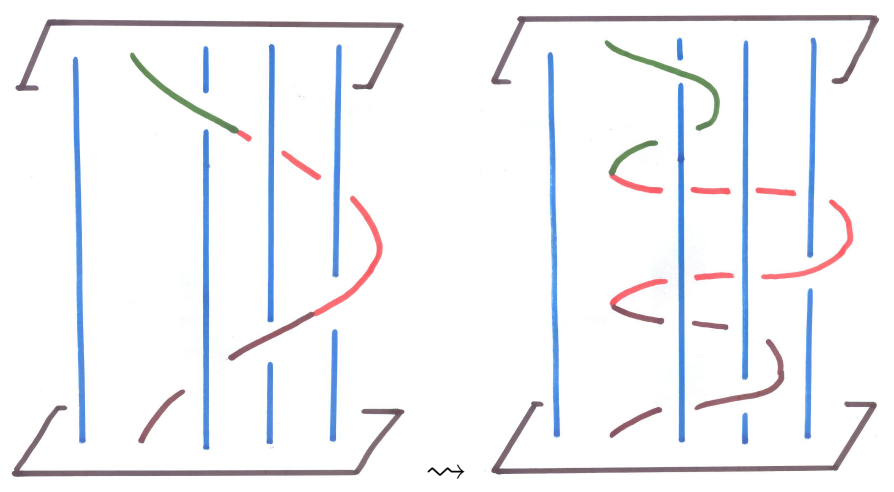

Figura 3.11: trança penteada como produto de $a_{i, j}$

As figuras 3.12, 3.13, 3.14, 3.15 e 3.16 nos mostram que valem as seguintes relações em $P_{n}\left(\mathbb{R}^{2}\right)$ (o símbolo $\rightleftharpoons$ significa que os elementos comutam):

(a) $a_{r, s} \rightleftharpoons a_{i, j}$, se $r<s<i<j$;

(b) $a_{r, s} \rightleftharpoons a_{i, j}$, se $i<r<s<j$;

(c) $a_{r, s} a_{r, j} a_{r, s}^{-1}=a_{s, j}^{-1} a_{r, j} a_{s, j}$, se $r<s<j$;

(d) $a_{r, s} a_{s, j} a_{r, s}^{-1}=a_{s, j}^{-1} a_{r, j}^{-1} a_{s, j} a_{r, j} a_{s, j}$, se $r<s<j$;

(e) $a_{i, j}^{-1} a_{s, j} a_{i, j} \rightleftharpoons a_{r, i}$, se $r<s<i<j$.

Estas relações definem um sistema completo de relações para $P_{n}\left(\mathbb{R}^{2}\right)$, isto é, todas as outras relações são provenientes destas. Mostremos que a relação (e) é equivalente a relação (e') $a_{r, i} a_{s, j} a_{r, i}^{-1}=a_{i, j}^{-1} a_{r, j}^{-1} a_{i, j} a_{r, j} a_{s, j} a_{r, j}^{-1} a_{i, j}^{-1} a_{r, j} a_{i, j}$, se $r<s<i<j$. De fato, 

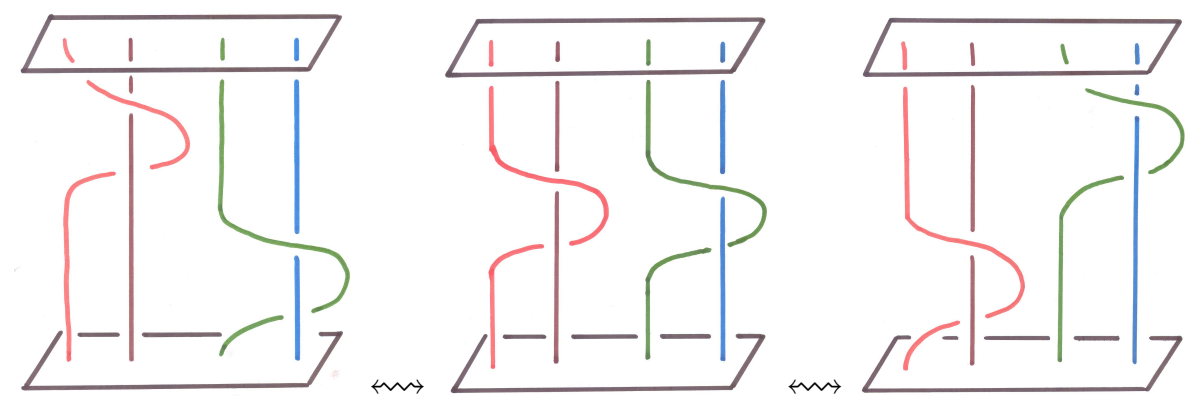

Figura 3.12: relação (a)
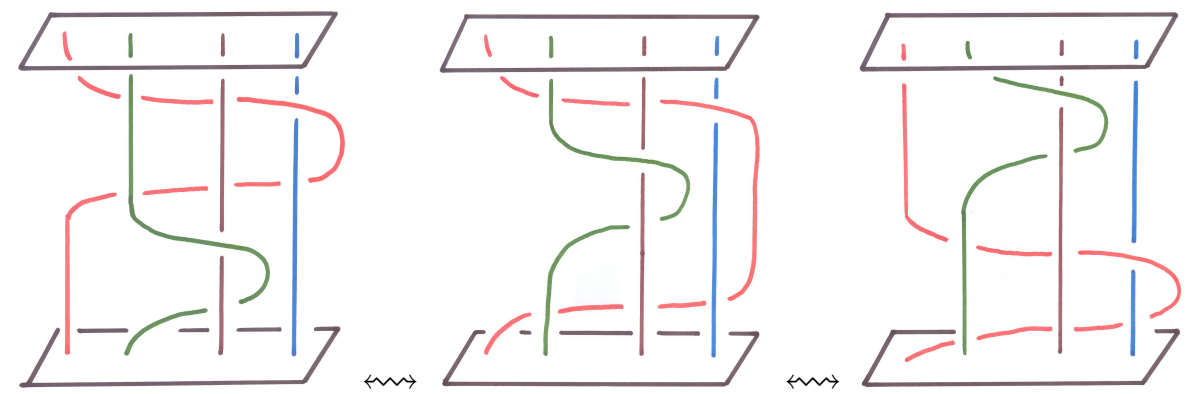

Figura 3.13: relação (b)

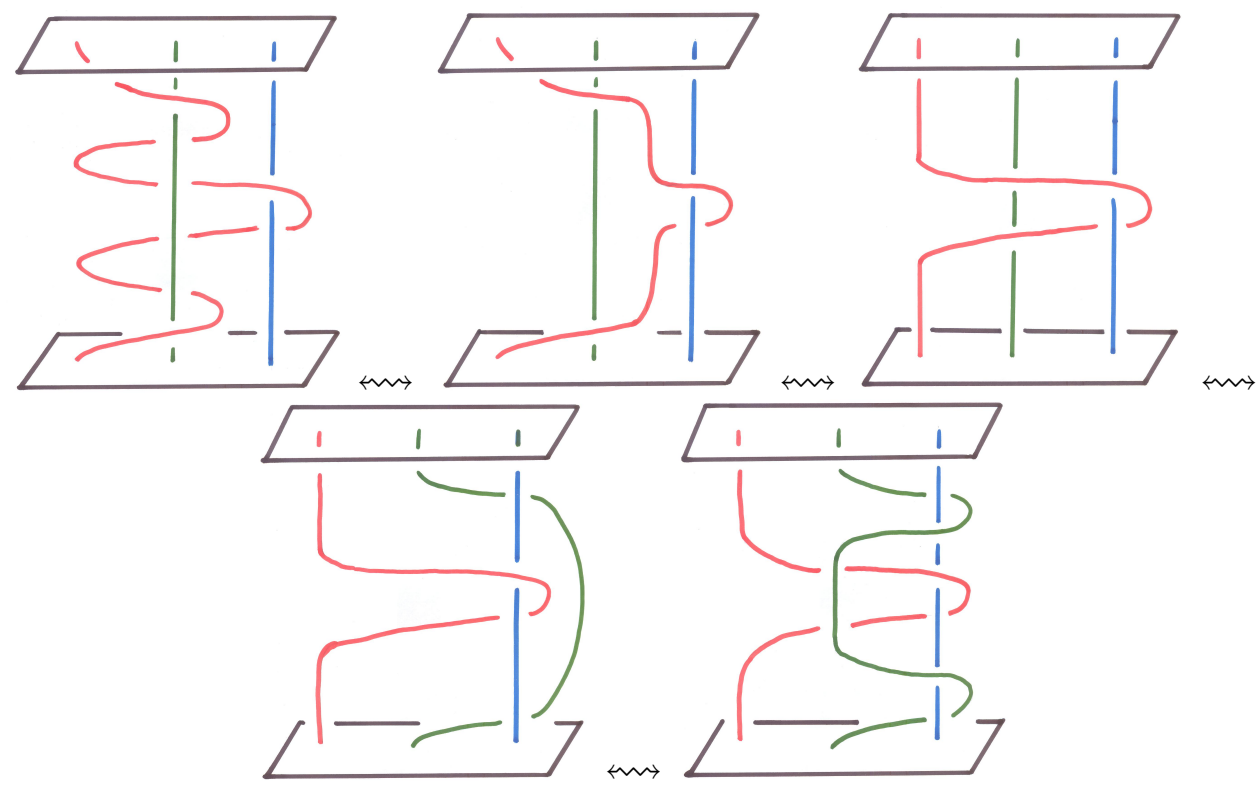

Figura 3.14: relação (c) 


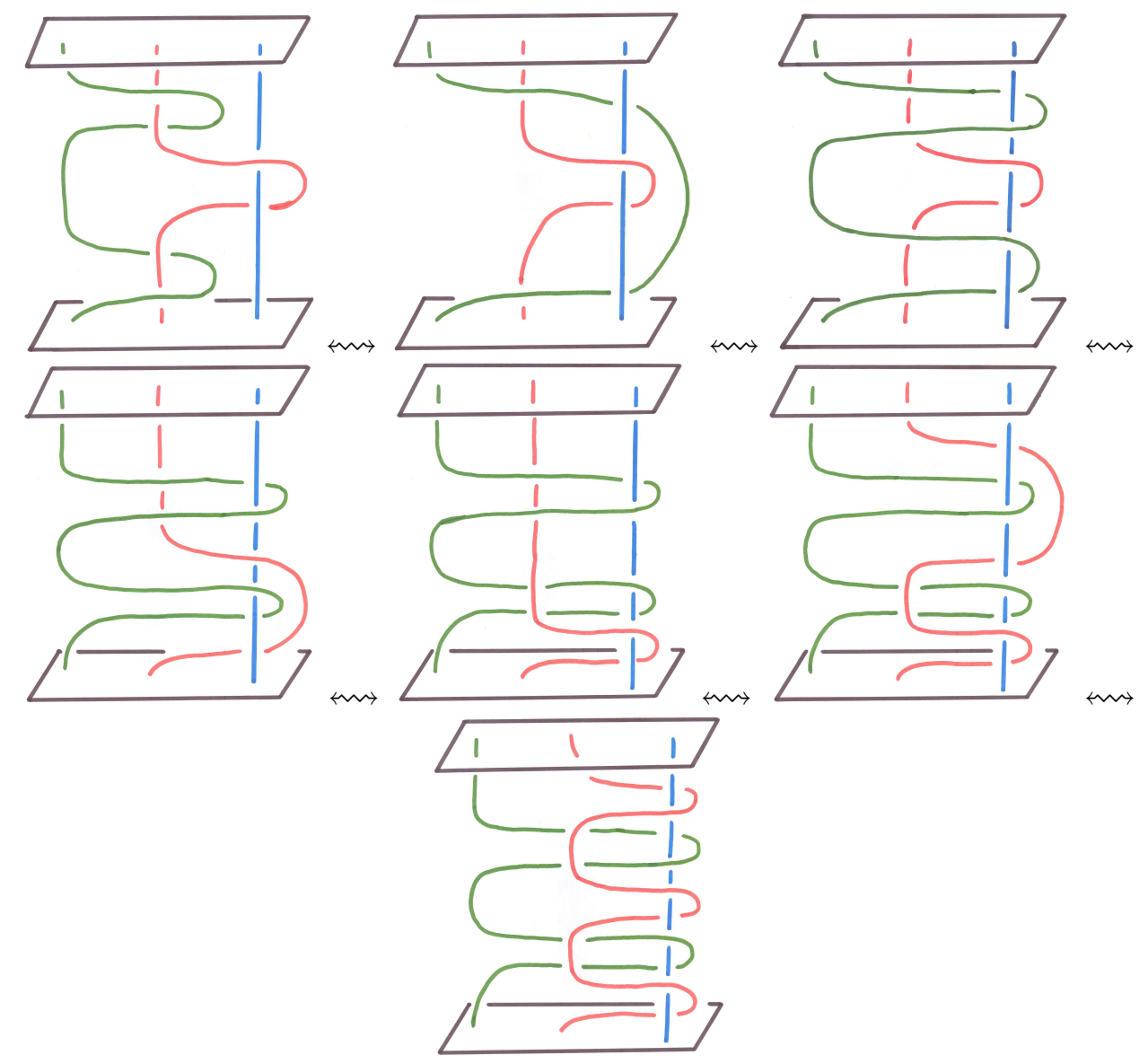

Figura 3.15: relação (d)

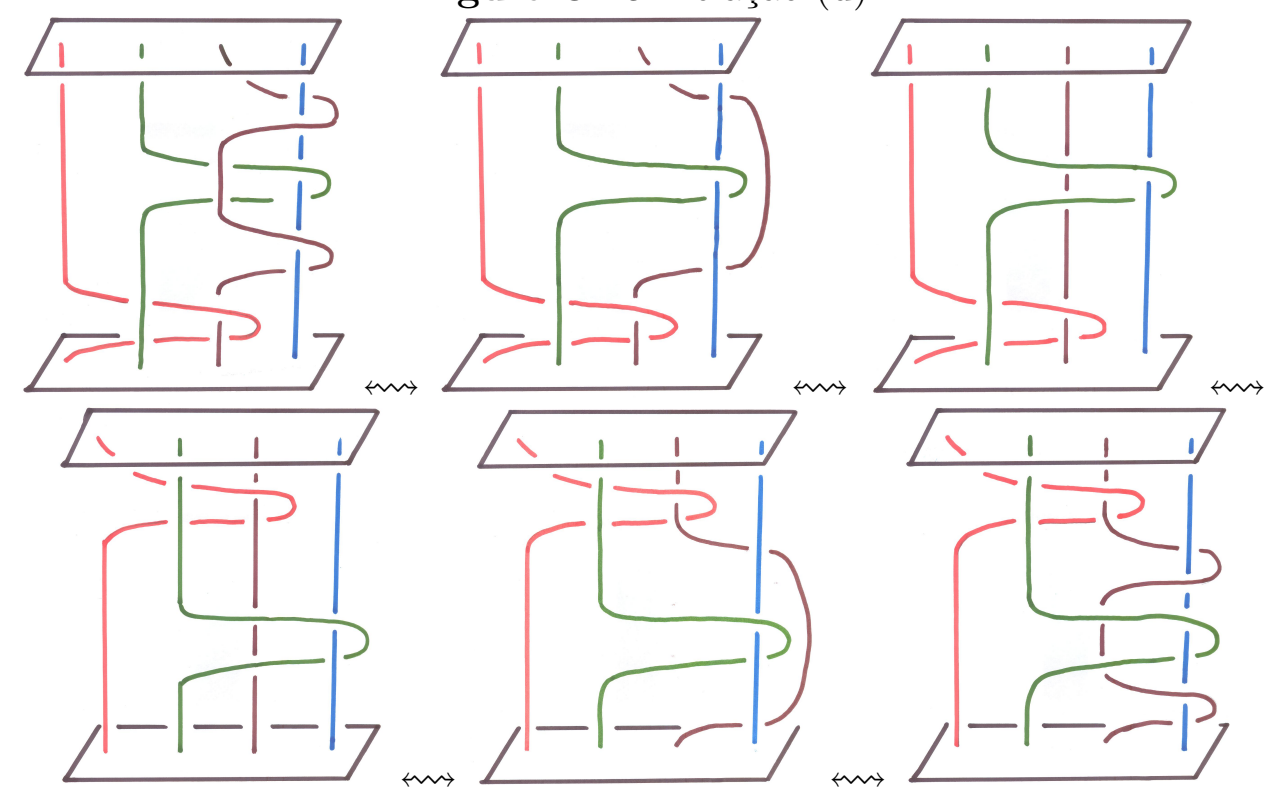

Figura 3.16: relação (e) 


$$
\begin{gathered}
(\mathrm{e}) \Leftrightarrow a_{r, i} a_{i, j}^{-1} a_{s, j} a_{i, j}=a_{i, j}^{-1} a_{s, j} a_{i, j} a_{r, i} \\
\mathbb{1} \\
a_{r, i} a_{i, j}^{-1} a_{s, j} a_{i, j} a_{r, i}^{-1}=a_{i, j}^{-1} a_{s, j} a_{i, j} \\
\mathbb{1} \\
\left(a_{r, i} a_{i, j}^{-1} a_{r, i}^{-1}\right) a_{r, i} a_{s, j} a_{r, i}^{-1}\left(a_{r, i} a_{i, j} a_{r, i}^{-1}\right)=a_{i, j}^{-1} a_{s, j} a_{i, j} \\
\mathbb{1} \\
a_{r, i} a_{s, j} a_{r, i}^{-1}=\left(a_{r, i} a_{i, j} a_{r, i}^{-1}\right) a_{i, j}^{-1} a_{s, j} a_{i, j}\left(a_{r, i} a_{i, j}^{-1} a_{r, i}^{-1}\right) \\
\mathbb{\mathbb { I }}(\mathrm{d}) \\
a_{r, i} a_{s, j} a_{r, i}^{-1}=\left(a_{i, j}^{-1} a_{r, j}^{-1} a_{i, j} a_{r, j} a_{i, j}\right) a_{i, j}^{-1} a_{s, j} a_{i, j}\left(a_{i, j}^{-1} a_{r, j}^{-1} a_{i, j}^{-1} a_{r, j} a_{i, j}\right) \Leftrightarrow\left(\mathrm{e}^{\prime}\right) .
\end{gathered}
$$

Renomeando os índices, podemos resumir estas relações de acordo com o

Teorema 3.3.1. $P_{n}\left(\mathbb{R}^{2}\right)$ admite a seguinte apresentação:

Geradores: $a_{i, j}, 1 \leq i<j \leq n$

Relações:

$$
a_{r, s} a_{i, j} a_{r, s}^{-1}=\left\{\begin{array}{l}
a_{i, j}, \text { se } i<r<s<j \text { ou } r<s<i<j \\
a_{i, j}^{-1} a_{r, j}^{-1} a_{i, j} a_{r, j} a_{i, j}, \text { se } r<i=s<j \\
a_{s, j}^{-1} a_{i, j} a_{s, j}, \text { se } i=r<s<j \\
a_{s, j}^{-1} a_{r, j}^{-1} a_{s, j} a_{r, j} a_{i, j} a_{r, j}^{-1} a_{s, j}^{-1} a_{r, j} a_{s, j}, \text { se } r<i<s<j
\end{array}\right.
$$

\section{4 $B_{n}\left(\mathbb{R}^{2}\right)$ como subgrupo de $B_{n}(M)$}

Em toda esta seção, $M$ denotará um superfície topológica conexa, compacta e sem bordo.

Seja $e: \mathbb{R}^{2} \rightarrow M$ um mergulho topológico. Então, $e$ induz um mergulho $\bar{e}$ : $\mathbb{R}^{2} \times[0,1] \rightarrow M \times[0,1]$, dado por $\bar{e}(x, t)=(e(x), t)$. Este mergulho por sua vez, induz um homomorfismo $\tilde{e}_{n}: B_{n}\left(\mathbb{R}^{2}\right) \rightarrow B_{n}(M)$, dado por

$$
\tilde{e}_{n}\left(b=\left(b_{1}, \ldots, b_{n}\right)\right)=\left(\bar{e} \circ b_{1}, \ldots, \bar{e} \circ b_{n}\right)
$$

O principal resultado desta seção é mostrar que $\tilde{e}_{n}$ é injetora se $M \neq \mathbb{S}^{2}, \mathbb{R P}^{2}$.

Em todo o restante desta dissertação, $\tilde{e}_{n}$ denotará este homomorfismo.

Vamos redefinir o homomorfismo $\tilde{e}_{n}$ em termos de Espaços de Configuração e fixar algumas notações para demonstrar o resultado.

Novamente, sendo $M$ um superfície topológia, fixemos um mergulho topológico $e: \mathbb{R}^{2} \rightarrow M$.

Seja $Q_{m}=\left\{q_{1}, \ldots, q_{m}\right\} \subset e\left(\mathbb{R}^{2}\right)$ um conjunto de $m$ pontos distintos de $M$. Consideremos $F_{m, n}(M)=F_{n}\left(M-Q_{m}\right)$ e $F_{m, n}\left(\mathbb{R}^{2}\right)=F_{n}\left(\mathbb{R}^{2}-e^{-1}\left(Q_{m}\right)\right)$. 
É fácil ver que a aplicação

$$
e_{m, n}: F_{m, n}\left(\mathbb{R}^{2}\right) \rightarrow F_{m, n}(M)
$$

definida por

$$
e_{m, n}\left(x_{1}, \ldots, x_{n}\right)=\left(e\left(x_{1}\right), \ldots, e\left(x_{n}\right)\right)
$$

é um mergulho topológico.

Quando $m=0, e_{m, n}=e_{0, n}$ induz um bem definido mergulho topológico $e_{n}$ : $C_{n}\left(\mathbb{R}^{2}\right) \rightarrow C_{n}(M)$, dado por $e_{n}\left(\left[\left(x_{1}, \ldots, x_{n}\right)\right]\right)=\left[e_{0, n}\left(x_{1}, \ldots, x_{n}\right)\right]$, que torna comutativo o seguinte diagrama:

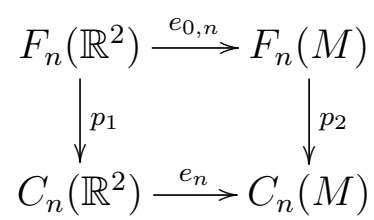

sendo $p_{1}$ e $p_{2}$ as projeções naturais.

A induzida em nível de $\pi_{1}$ de $e_{n}$ é o homomorfismo $\tilde{e}_{n}: B_{n}\left(\mathbb{R}^{2}\right) \rightarrow B_{n}(M)$ e o resultado principal desta seção é o

Teorema 3.4.1. Se $M \neq \mathbb{S}^{2}, \mathbb{R P}^{2}$, então $\tilde{e}_{n}$ é injetora para todo $n$.

Aplicando o funtor $\pi_{1}$ no diagrama anterior, e lembrando que $P_{n}(M)=\pi_{1}\left(F_{n}(M)\right)$ e $B_{n}(M)=\pi_{1}\left(C_{n}(M)\right)$, temos o seguinte diagrama comutativo:

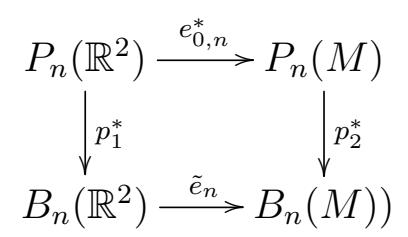

Notemos que se $\beta \in B_{n}\left(\mathbb{R}^{2}\right)$, então $\alpha(\beta)=\alpha\left(\tilde{e}_{n}(\beta)\right)$, pela própria definição de $\tilde{e}_{n}$. Logo, se $\beta \notin P_{n}\left(\mathbb{R}^{2}\right)$, então $\alpha\left(\tilde{e}_{n}(\beta)\right)$ não é a permutação trivial. Notemos ainda que $\tilde{e}_{n}$ restrita a $P_{n}\left(\mathbb{R}^{2}\right)$ pode ser considerada como $e_{0, n}^{*}$ pelo que já foi discutido no capítulo 2. Logo, temos o

Teorema 3.4.2. $\tilde{e}_{n}: B_{n}\left(\mathbb{R}^{2}\right) \rightarrow B_{n}(M)$ é injetor se, e somente se, $e_{0, n}^{*}: P_{n}\left(\mathbb{R}^{2}\right) \rightarrow$ $P_{n}(M)$ é injetor.

Portanto, para provar o teorema 3.4.1, basta que provemos o

Teorema 3.4.3. Se $M \neq \mathbb{S}^{2}, \mathbb{R P}^{2}$, então $e_{0, n}^{*}: P_{n}\left(\mathbb{R}^{2}\right) \rightarrow P_{n}(M)$ é injetor para todo $n$.

Antes de iniciarmos a demonstração, vamos provar alguns resultados. 
Lema 3.4.4. Se $M \neq \mathbb{S}^{2}, \mathbb{R}^{2}$, então $\pi_{2}\left(M-Q_{m}\right)=\pi_{3}\left(M-Q_{m}\right)=1$ para todo $m \geq 0$.

Prova: Se $m=0$, então $M-Q_{m}=M$. Como $M \neq \mathbb{S}^{2}, \mathbb{R}^{2}$, então o recobrimento universal de $M$ é o $\mathbb{R}^{2}$, o qual é contrátil. Como uma aplicação de recobrimento induz um isomorfismo nos grupos de homotopia de dimensão maior ou igual que 2 , segue que $\pi_{3}(M)=\pi_{2}(M)=1$. Se $m \neq 0$, pelo teorema de classificação de superfícies, $M-Q_{m}$ é homeomorfo a um polígono do plano menos uma quantidade $m$ de pontos de seu interior, passado a quociente onde os vértices estão todos identificados e as arestas estão identificadas aos pares de modo conveniente. Agora, todo espaço desta forma tem o mesmo tipo de homotopia que um grafo. A figura 3.17 dá uma ideia deste fato quando $M$ é a superfície orientável de gênero 2 (bitoro) e claramente este argumento se generaliza para as outras superfícies. O resultado segue agora do fato que
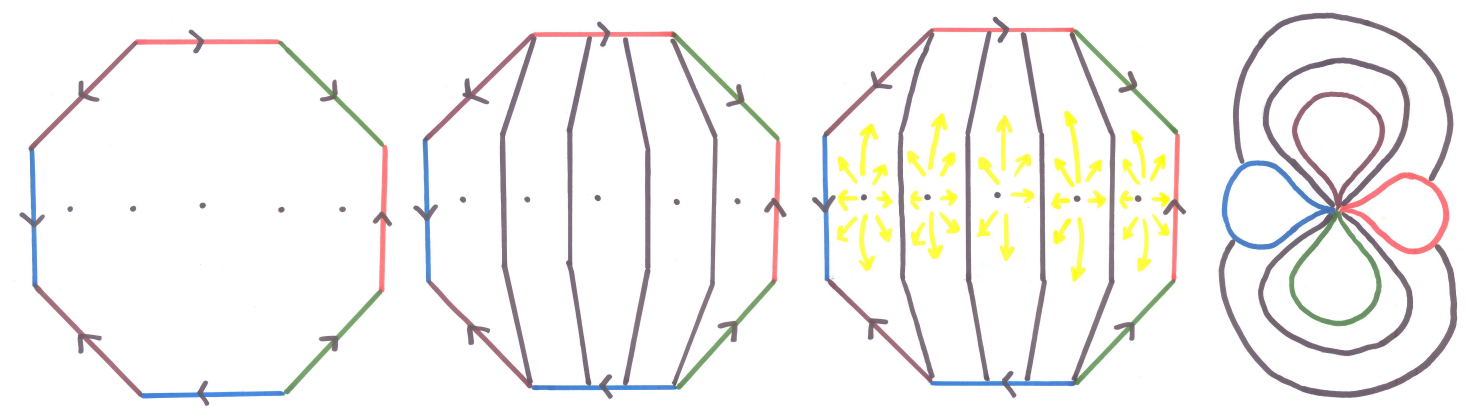

Figura 3.17: deformação de $M-Q_{m}$ num grafo

o recobrimento universal de um grafo é uma árvore, que é sempre contrátil e novamente, um recobrimento induz isomorfismo nos grupos de homotopia de dimensão maior ou igual que 2 .

Lema 3.4.5. Se $M \neq \mathbb{S}^{2}, \mathbb{R P}^{2}$, então $\pi_{2}\left(F_{n}(M)\right)=1$ para todo $n \geq 1$.

Prova: Se $n=1$, o resultado segue direto do lema 3.4 .4 e do fato que $F_{1}(M)=M$. Suponhamos então $n>1$. Consideremos a fibração $\pi: F_{m, n}(M) \rightarrow F_{m, 1}(M)=$ $M-Q_{m}$, definida no teorema de Fadell e Neuwirth (teo 1.2.2), cuja fibra típica é $F_{m+1, n-1}(M)$. A sequência exata de homotopia associada a $\pi$ é

$$
\ldots \quad \pi_{3}\left(M-Q_{m}\right) \longrightarrow \pi_{2}\left(F_{m+1, n-1}(M)\right) \longrightarrow \pi_{2}\left(F_{m, n}(M)\right) \longrightarrow \pi_{2}\left(M-Q_{m}\right) \longrightarrow \ldots
$$

Pelo lema 3.4.4, segue que $\pi_{2}\left(F_{m+1, n-1}(M)\right) \approx \pi_{2}\left(F_{m, n}(M)\right)$.

Novamente, pelo lema 3.4.4 temos que 
$1=\pi_{2}\left(M-Q_{n-1}\right)=\pi_{2}\left(F_{n-1,1}(M)\right) \stackrel{*}{\approx} \pi_{2}\left(F_{n-2,2}(M)\right) \stackrel{*}{\approx} \ldots \stackrel{*}{\approx} \pi_{2}\left(F_{0, n}(M)\right)=\pi_{2}\left(F_{n}(M)\right)$

Segue o resultado.

Observação 3.4.6. O lema 3.4.4 também vale para o caso da superfície $M$ ser o $\mathbb{R}^{2}$. De fato, para $m=0$ é óbvio e $\mathbb{R}^{2}$ menos uma quantidade finita de pontos tem o mesmo tipo de homotopia que a figura oito generalizada, que é um grafo. Veja a figura 3.18. O restante da argumentação é idêntica ao do lema 3.4.4. Assim, a mesma argumentação do lema 3.4.5 vale para $\mathbb{R}^{2}$ e portanto $\pi_{2}\left(F_{n}\left(\mathbb{R}^{2}\right)\right)=1$ para todo $n \geq 1$.

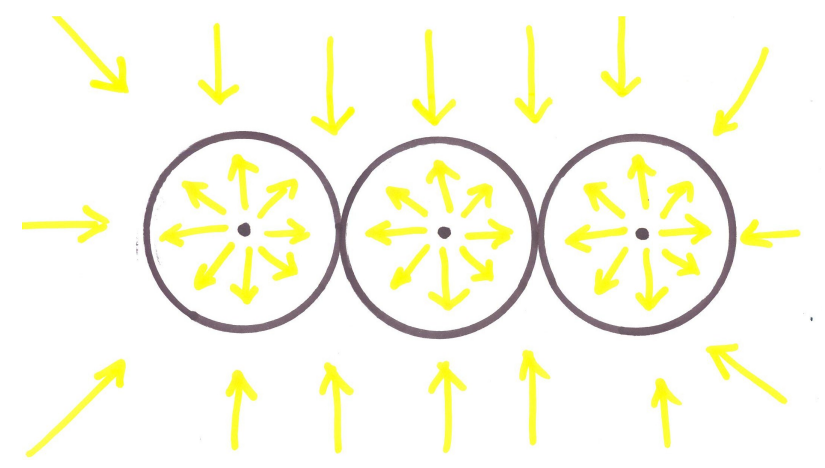

Figura 3.18: $\mathbb{R}^{2}$ menos uma quantidade finita de pontos deformando na figura oito generalizada

Lema 3.4.7. Se $M \neq \mathbb{S}^{2}, \mathbb{R P}^{2}$, então $e_{m, 1}^{*}: \pi_{1}\left(\mathbb{R}^{2}-e^{-1}\left(Q_{m}\right)\right) \rightarrow \pi_{1}\left(M-Q_{m}\right)$ é injetora para todo $m \geq 0$, sendo $e_{m, 1}^{*}$ a induzida do mergulho $e_{m, 1}$.

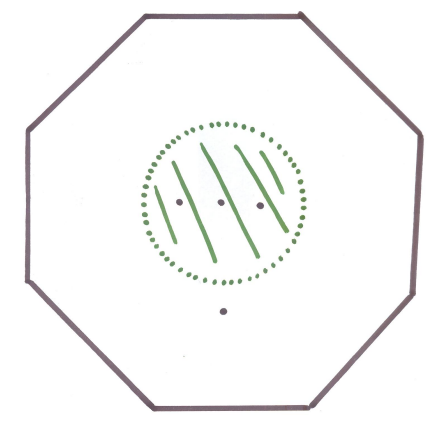

Figura 3.19: Aberto $U$

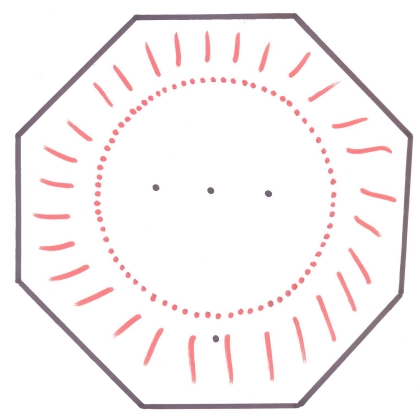

Figura 3.20: Aberto V

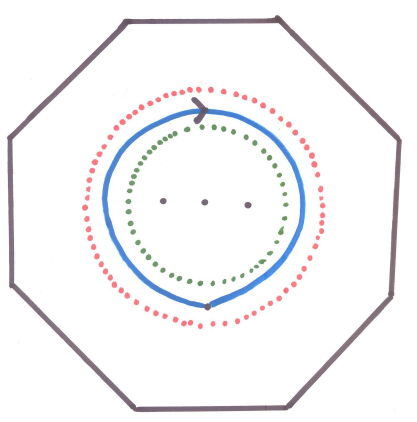

Figura 3.21: gerador de $\pi_{1}(U \cap V)$

Prova: Se $m=0$, então a afirmação é verdadeira, pois $Q_{m}=\emptyset$ e $\pi_{1}\left(\mathbb{R}^{2}\right)=1$. Suponhamos então $m>0$. Pelo teorema de classificação de superfícies, consideremos $M-$ 
$Q_{m}$ o espaço quociente de um polígono menos uma quantidade $m$ de pontos do interior deste polígono, segundo o qual os lados que constituem o bordo estão identificados dois a dois e todos os vértices estão identificados em um único ponto. Consideremos em $M-Q_{m}$ os abertos $U$ e $V$, conforme as figuras 3.19 e 3.20 respectivamente. Notemos que o aberto $V$ tem o mesmo tipo de homotopia que um bouquet de $S^{1}$. A figura 3.22 nos dá uma ideia deste fato quando $M$ é o bitoro, mas é fácil ver que este argumento vale para todas as superfícies. Logo, $\pi_{1}(V)$ é livre de rank finito, digamos $k$ (este $k$ depende do gênero da superfície e se ela é orientável ou não). Denotemos por $y_{1}, \ldots, y_{k}$ os geradores deste grupo. Agora notemos que $\pi_{1}(U)$ é livre de rank $m$ e que os geradores são loops que circulam cada ponto retirado de $M$, digamos $x_{1}, \ldots, x_{m}$. Na figura 3.21 está representado um gerador de $\pi_{1}(U \cap V)$ (é fácil ver que $U \cap V$ tem o mesmo tipo de homotopia que $S^{1}$ ). Como $M \neq \mathbb{S}^{2}, \mathbb{R P}^{2}$, a inclusão deste gerador em $\pi_{1}(V)$ é um elemento não nulo, ou seja, é uma palavra não nula em termos dos geradores $y_{1}, \ldots, y_{k}$, digamos $y$. Já a inclusão em $\pi_{1}(U)$ é igual ao produto dos geradores numa certa ordem e sem perda de generalidade, podemos supor que seja $x_{1} \ldots x_{m}$. Logo, pelo teorema de Van Kampen, $\pi_{1}\left(M-Q_{m}\right)=\left\langle y_{1}, \ldots, y_{k}, x_{1}, \ldots, x_{m} \mid y=x_{1} \ldots x_{m}\right\rangle$. Sejam $x_{1}^{\prime}, \ldots x_{m}^{\prime}$ os geradores do grupo livre $\pi_{1}\left(\mathbb{R}^{2}-Q_{m}\right)$. Pela definição do mergulho $e_{m, 1}$, sem perda de generalidade, podemos supor que $e_{m, 1}^{*}\left(x_{i}^{\prime}\right)=x_{i}$, para todo $i=1, \ldots, m$. Logo, para mostrarmos que $e_{m, 1}^{*}$ é injetora, basta mostrarmos que o subgrupo de $\pi_{1}\left(M-Q_{m}\right)$ gerado por $x_{1}, \ldots, x_{m}$ é livre de rank $m$. Ora, mas se este subgrupo não fosse livre, então existiria uma relação não trivial envolvendo apenas os geradores $x_{1}, \ldots, x_{m}$ de $\pi_{1}\left(M-Q_{m}\right)$, mas não existe de acordo com a apresentação que obtivemos, e assim, concluímos que

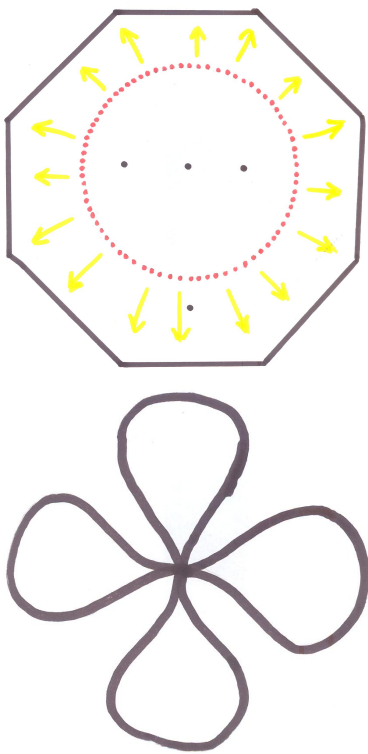

Figura 3.22:

bitoro deformando no bouquet de $S^{1}$ $e_{m, 1}^{*}$ é injetora.

Provados estes lemas, vamos a

Prova:[teorema 3.4.3] Seja $n \geq 1$ e sejam $x=\left(x_{1}^{0}, x_{2}^{0}, \ldots, x_{n+1}^{0}\right) \in F_{0, n+1}\left(\mathbb{R}^{2}\right), x^{\prime}=$ $\left(x_{2}^{0}, \ldots, x_{n+1}^{0}\right)$ e $Q_{n}=\left\{e\left(x_{2}^{0}\right), \ldots, e\left(x_{n+1}^{0}\right)\right\}$. Notemos que é comutativo o seguinte diagrama:

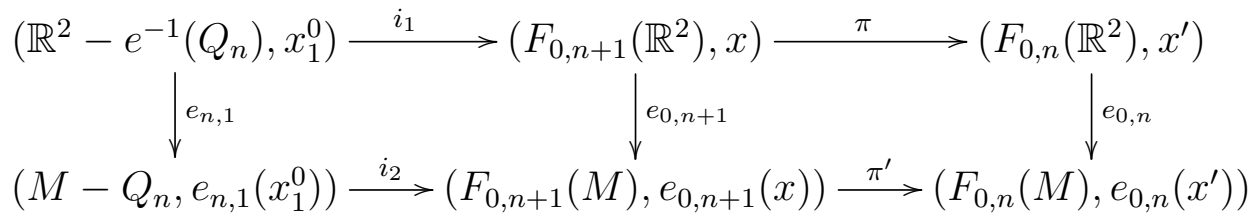


sendo $i_{1}: \mathbb{R}^{2}-e^{-1}\left(Q_{n}\right) \rightarrow F_{0, n+1}\left(\mathbb{R}^{2}\right)$ e $i_{2}: M-Q_{n} \rightarrow F_{0, n+1}(M)$ definidas por

$$
i_{1}(a)=\left(a, x_{2}^{0}, \ldots, x_{n+1}^{0}\right) \text { e } i_{2}(b)=\left(b, e\left(x_{2}^{0}\right), \ldots, e\left(x_{n+1}^{0}\right)\right)
$$

e as funções $\pi$ e $\pi^{\prime}$ são fibrações definidas no teorema 1.2.2 (Fadell e Neuwirth). Passando o funtor $\pi_{1}$ neste diagrama e colocando os grupos triviais nas pontas, temos o seguinte diagrama comutativo (omitimos os pontos base):

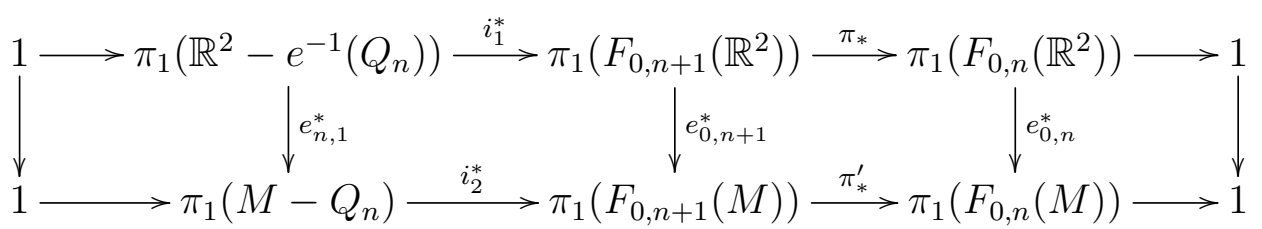

Como $M-Q_{n}$ e $\mathbb{R}^{2}-e^{-1}\left(Q_{n}\right)$ são conexos por caminhos, então $\pi_{0}\left(M-Q_{n}\right)=\pi_{0}\left(\mathbb{R}^{2}-\right.$ $\left.e^{-1}\left(Q_{n}\right)\right)=1$. Pelo lema 3.4 .5 e pela observação $3.4 .6, \pi_{2}\left(F_{0, n}(M)\right)=\pi_{2}\left(\mathbb{R}^{2}\right)=1$. Portanto, as sequências horizontais do diagrama acima são parte das sequências exatas de homotopia associada as fibrações $\pi$ e $\pi^{\prime}$. Pelo lema 3.4.7, $e_{n, 1}^{*}$ é injetora. Usando indução em $n$ e o lema dos cinco o teorema estará provado se $e_{0,1}^{*}: \pi_{1}\left(\mathbb{R}^{2}\right) \rightarrow \pi_{1}(M)$ for injetora. Mas isto segue diretamente do fato que $\pi_{1}\left(\mathbb{R}^{2}\right)=1$.

Observemos que no caso $n=1$, $\tilde{e}_{1}$ é injetora mesmo

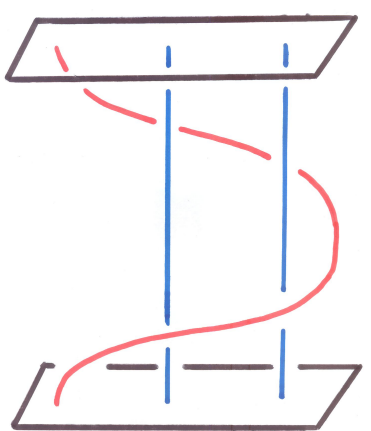

Figura 3.23: 3-trança $b$ quando $M$ é igual a $\mathbb{S}^{2}$ ou $\mathbb{R P}^{2}$, pois $C_{1}\left(\mathbb{R}^{2}\right)=F_{1}\left(\mathbb{R}^{2}\right)=$ $\mathbb{R}^{2}$ e $\pi_{1}\left(\mathbb{R}^{2}\right)=1$. No caso de $n>1$, os argumentos não valem para estes casos, pois usamos o lema 3.4.4 para provar o teorema 3.4 .3 e $\pi_{2}\left(\mathbb{S}^{2}\right)$ e $\pi_{2}\left(\mathbb{R} \mathbb{P}^{2}\right)$ são isomorfos ao grupo cíclico de ordem infinita. O lema 3.4.4 é um resultado fundamental para provar o teorema 3.4.3, pois vale uma recíproca quando $n>1$. Para provar este fato, basta exibirmos elementos de $P_{n}\left(\mathbb{R}^{2}\right)$ não nulos, cuja imagem por $\tilde{e}_{n}$ em $P_{n}\left(\mathbb{S}^{2}\right)$ e $P_{n}\left(\mathbb{R P}^{2}\right)$ sejam nulos. Consideremos em $P_{n}\left(\mathbb{R}^{2}\right)$ a trança $b=\sigma_{1}^{-1} \ldots \sigma_{n-2}^{-1} \sigma_{n-1}^{2} \sigma_{n-2}^{-1} \ldots \sigma_{1}^{-1}$. A figura 3.23 mostra um esquema desta trança quando $n=3$ e para os outros casos, basta retirarmos ou colocarmos mais segmentos verticais entre a primeira e última corda. Claramente, esta trança não se deforma na trança trivial. Entretanto, esta trança, considerada com elemento de $P_{n}\left(\mathbb{S}^{2}\right)$ via o homomorfismo $\tilde{e}_{n}$ se deforma na trança trivial. A figura 3.24 mostra esta deformação quando $n=3$, e novamente, o argumento se generaliza para os outros casos, colocanco ou retirando segmentos verticais entre a primeira e a última corda. O caso do $\mathbb{R P}^{2}$ é um pouco mais sutil. Em [9], é exibido um elemento não trivial de $P_{n}\left(\mathbb{R}^{2}\right)$, que se deforma na identidade quando considerado em $P_{n}\left(\mathbb{R P}^{2}\right)$. 


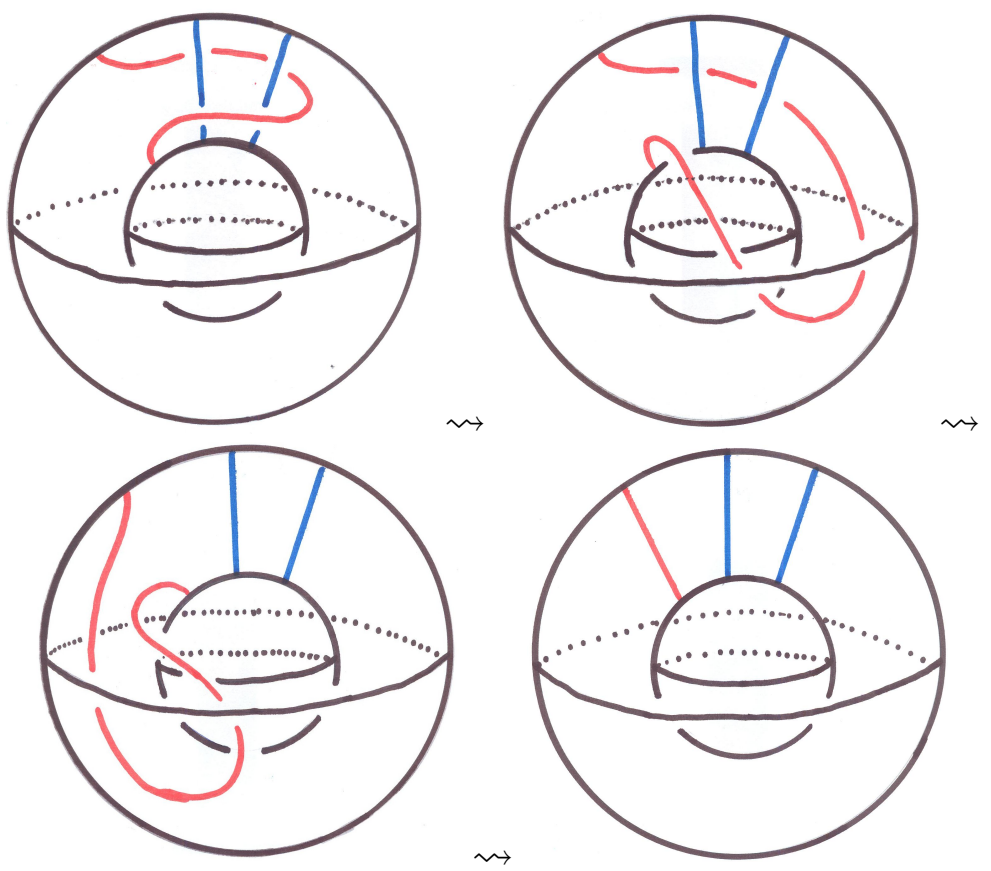

Figura 3.24: deformação de 3-trança de $\mathbb{S}^{2}$ na identidade 



\begin{tabular}{|c|c|}
\hline & Capítulo \\
\cline { 2 - 2 } & 4 \\
\hline
\end{tabular}

\section{Grupos de Tranças do Plano Projetivo}

\subsection{Introdução}

Em todo este capítulo, vamos considerar $\mathbb{R P}^{2}$ como o disco fechado unitário centrado na origem de $\mathbb{R}^{2}$, passado a quociente pela relação de equivalência que identifica pontos antípodas do bordo, salvo quando for explicitado outra descrição.

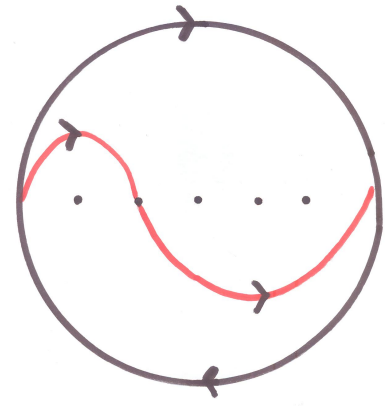

Figura 4.1: imagem do caminho $\overline{\rho_{i}}$

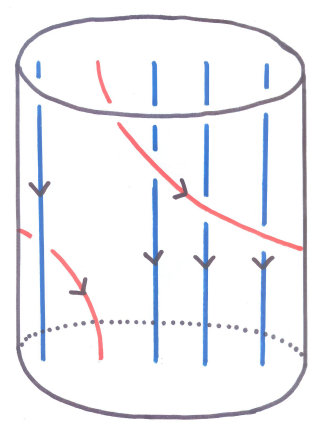

Figura 4.2:

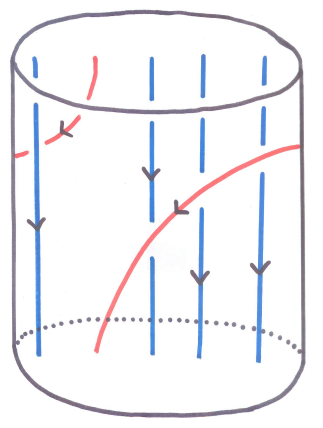

Figura 4.3:

trança $\rho_{i}^{-1}$

Sejam $\{(1,0), \ldots,(n, 0)\} \subset \mathbb{R}^{2}, c=\left(\frac{n+1}{2}, 0\right) \in \mathbb{R}^{2}$ e $B$ o disco aberto de centro $c$ e raio $\frac{n+1}{2}$ (o qual contém os pontos $(1,0), \ldots,(n, 0)$ ). Seja $f: \mathbb{R}^{2} \rightarrow B$ um mergulho 
simples e $g: B \rightarrow \mathbb{R P}^{2}$ o mergulho que aplica $B$ no disco aberto unitário contido em $\mathbb{R P}^{2}$. Definimos o mergulho $e: \mathbb{R}^{2} \rightarrow \mathbb{R P}^{2}$, por $e(x)=g \circ f(x)$.

Chamaremos as imagens das tranças de Artin pelo homomorfismo $\tilde{e}_{n}$ também de tranças de Artin, e também as denotaremos por $\sigma_{i}, i=1, \ldots, n-1$.

Diferente de $B_{n}\left(\mathbb{R}^{2}\right)$, as tranças de Artin não geram todo $B_{n}\left(\mathbb{R P}^{2}\right)$.

Fixemos $i \in\{1, \ldots, n\}$. Seja $p_{i}=e(i, 0)$ e $\overline{\rho_{i}}:[0,1] \rightarrow \mathbb{R P}^{2}$ um caminho injetor como na figura 4.1 (na figura, $n=5$ e $i=2$ ).

Definimos $\rho_{i}=\left(b_{1}, \ldots, b_{n}\right) \in B_{n}\left(\mathbb{R P}^{2}\right)$ a trança tal que $b_{j}:[0,1] \rightarrow \mathbb{R P}^{2} \times[0,1]$ são os caminhos definidos por

$$
b_{j}(t)=(e(j, 0), t), j \neq i \quad b_{i}(t)=\left(\overline{\rho_{i}}(t), t\right)
$$

Na figuras 4.2 e 4.3, estão representados esquemas das tranças $\rho_{i}$ e $\rho_{i}^{-1}$, onde $n=5$ e $i=2$, sendo que a linha vermelha representa a $i$-ésima corda e as linhas azuis representam as demais cordas.

As figuras 4.4 4.5, 4.6 e 4.7 mostram que além de valer as relações de Artin

(i) $\sigma_{i} \sigma_{j}=\sigma_{j} \sigma_{i}$, se $|i-j| \geq 2$, e

(ii) $\sigma_{i} \sigma_{i+1} \sigma_{i}=\sigma_{i+1} \sigma_{i} \sigma_{i+1}$

as tranças $\rho_{i}, i=1, \ldots, n$, juntas com as tranças de Artin, estão sujeitas as seguintes relações:

(iii) $\sigma_{i} \rho_{j}=\rho_{j} \sigma_{i}(j \neq i, i+1)$;

(iv) $\rho_{i}=\sigma_{i} \rho_{i+1} \sigma_{i}$;

(v) $\rho_{i+1}^{-1} \rho_{i}^{-1} \rho_{i+1} \rho_{i}=\sigma_{i}^{2}$;

(vi) $\rho_{1}^{2}=\sigma_{1} \sigma_{2} \ldots \sigma_{n-2} \sigma_{n-1}^{2} \sigma_{n-2} \ldots \sigma_{2} \sigma_{1}$.

O objetivo deste capítulo é mostrar que as tranças $\rho_{i}, i=1, \ldots, n$, juntos com as tranças de Artin, geram $B_{n}\left(\mathbb{R P}^{2}\right)$ e que as relações (i), (ii), (iii), (iv), (v) e (vi) formam um sistema completo de relações.

Consideremos $G_{n}\left(\mathbb{R P}^{2}\right)$ o grupo cuja apresentação é a apresentação que queremos obter para $B_{n}\left(\mathbb{R P}^{2}\right)$. Na seção 4.2 , vamos definir e extrair propriedades deste grupo, chamado grupo de tranças algébrico do plano projetivo.

Na seção 4.3, vamos extrair várias propriedades de $B_{n}\left(\mathbb{R P}^{2}\right)$.

Na seção 4.4, usando as informações das seções 4.2 e 4.4 , mostraremos que $G_{n}\left(\mathbb{R P}^{2}\right)$ é isomorfo a $B_{n}\left(\mathbb{R P}^{2}\right)$ através de um homomorfismo natural.

\subsection{Propriedades de $G_{n}\left(\mathbb{R P}^{2}\right)$}

Definição 4.2.1. O n-ésimo grupo algébrico de tranças do plano projetivo, denotado por $G_{n}\left(\mathbb{R P}^{2}\right)$, é o grupo que tem a seguinte apresentação: 

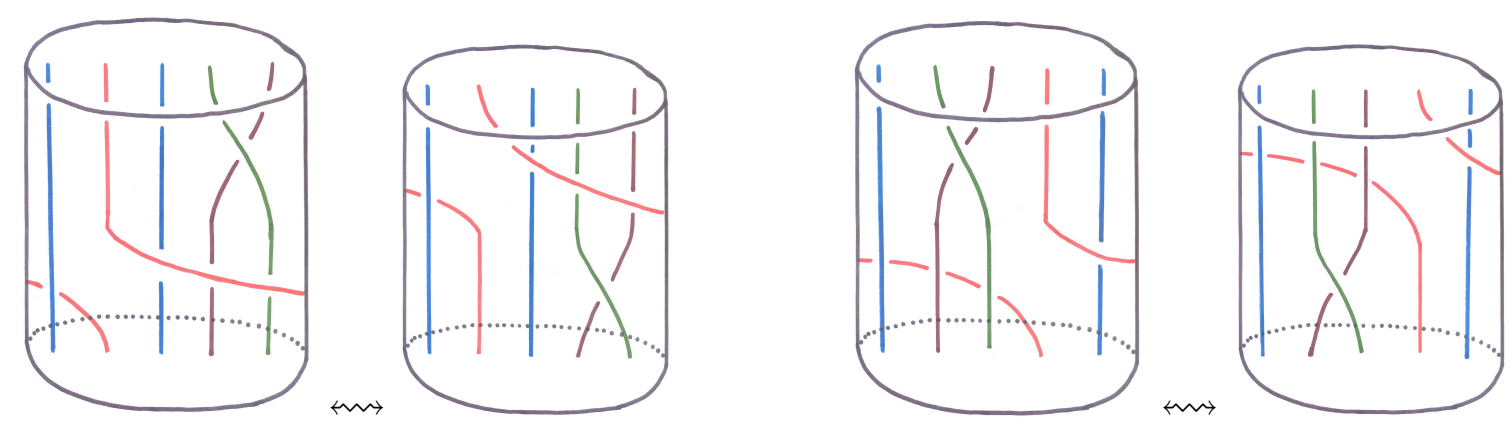

Figura 4.4: relação (iii)
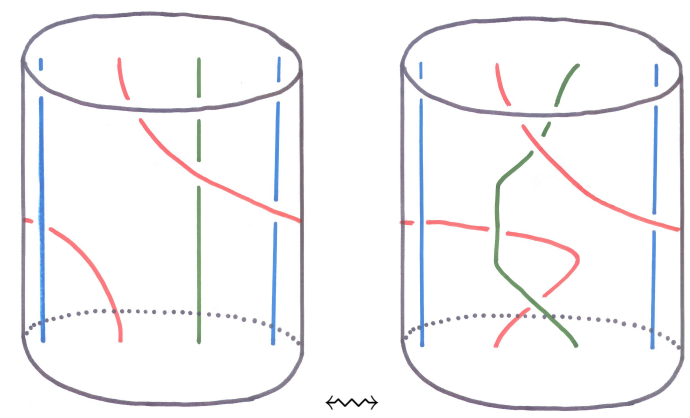

Figura 4.5: relação (iv)
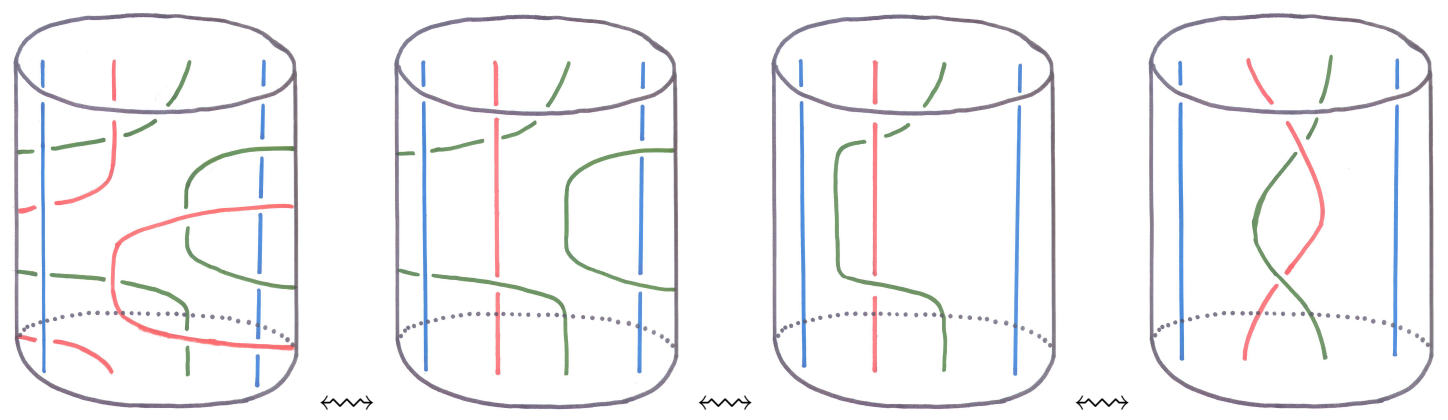

Figura 4.6: relação (v)
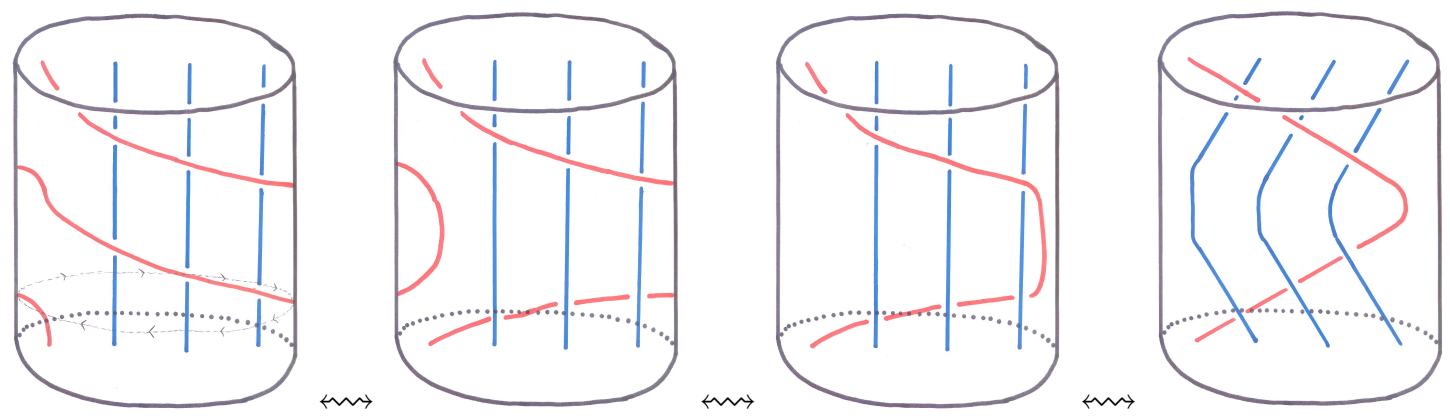

Figura 4.7: relação (vi) 
Geradores: $\tilde{\sigma}_{1}, \ldots, \tilde{\sigma}_{n-1}, \tilde{\rho}_{1}, \ldots, \tilde{\rho}_{n}$.

Relações: (i) $\tilde{\sigma}_{i} \tilde{\sigma}_{j}=\tilde{\sigma}_{j} \tilde{\sigma}_{i}$, se $|i-j| \geq 2$;

(ii) $\tilde{\sigma}_{i} \tilde{\sigma}_{i+1} \tilde{\sigma}_{i}=\tilde{\sigma}_{i+1} \tilde{\sigma}_{i} \tilde{\sigma}_{i+1}$;

(iii) $\tilde{\sigma}_{i} \tilde{\rho}_{j}=\tilde{\rho}_{j} \tilde{\sigma}_{i}$, se $j \neq i, i+1$;

(iv) $\tilde{\rho}_{i}=\tilde{\sigma}_{i} \tilde{\rho}_{i+1} \tilde{\sigma}_{i}$;

(v) $\tilde{\rho}_{i+1}^{-1} \tilde{\rho}_{i}^{-1} \tilde{\rho}_{i+1} \tilde{\rho}_{i}=\tilde{\sigma}_{i}^{2}$;

(vi) $\tilde{\rho}_{1}^{2}=\tilde{\sigma}_{1} \tilde{\sigma}_{2} \ldots \tilde{\sigma}_{n-2} \tilde{\sigma}_{n-1}^{2} \tilde{\sigma}_{n-2} \ldots \tilde{\sigma}_{2} \tilde{\sigma}_{1}$.

Seja $\gamma: G_{n}\left(\mathbb{R} \mathbb{P}^{2}\right) \rightarrow S_{n}$ o homomorfismo que mapeia $\tilde{\sigma}_{i}$ na transposição $(i, i+1), i=1, \ldots, n-1$, e mapeia $\tilde{\rho}_{j}$ na permutação identidade, $j=1, \ldots, n$, e estendido por linearidade. Cálculos simples mostram que $\gamma$ está bem definida, isto é, que $\gamma$ manda cada relação de $G_{n}\left(\mathbb{R P}^{2}\right)$ na permutação trivial. O homomorfismo $\gamma$ é chamado homomorfismo permutação de $G_{n}\left(\mathbb{R P}^{2}\right)$.

Consideremos $K_{n}\left(\mathbb{R P}^{2}\right)$ o núcleo do homomorfismo $\gamma$ e $D_{n}\left(\mathbb{R P}^{2}\right)=\{t \in$ $\left.G_{n}\left(\mathbb{R P}^{2}\right) \mid \gamma(t)(1)=1\right\}$, ou seja, $D_{n}\left(\mathbb{R} \mathbb{P}^{2}\right)$ é o subgrupo de $G_{n}\left(\mathbb{R P}^{2}\right)$ cujos elementos são mapeados por $\gamma$ nas permutações que deixam o número 1 fixo.

Teorema 4.2.2. Sejam $t_{1}, t_{2} \in G_{n}\left(\mathbb{R P}^{2}\right)$. Então $t_{1}$ e $t_{2}$ pertencem a mesma classe lateral a esquerda de $D_{n}\left(\mathbb{R P}^{2}\right)$ se, e somente se, $\left[\gamma\left(t_{1}\right)\right]^{-1}(1)=\left[\gamma\left(t_{2}\right)\right]^{-1}(1)$.

Prova: Sejam $t_{1}, t_{2} \in G_{n}\left(\mathbb{R P}^{2}\right)$. Suponhamos que $t_{1} D_{n}\left(\mathbb{R P}^{2}\right)=t_{2} D_{n}\left(\mathbb{R P}^{2}\right)$. Então, existe $t \in D_{n}\left(\mathbb{R P}^{2}\right)$, tal que $t_{1}=t_{2} t$. Aplicando o homomorfismo $\gamma$ nos dois lados da igualdade temos $\gamma\left(t_{1}\right)=\gamma\left(t_{2}\right) \gamma(t):=\gamma(t) \circ \gamma\left(t_{2}\right) \Rightarrow\left[\gamma\left(t_{2}\right)\right]^{-1}=\left[\gamma\left(t_{1}\right)\right]^{-1} \circ \gamma(t)$. Como $t \in D_{n}\left(\mathbb{R} \mathbb{P}^{2}\right)$, então $\gamma(t)(1)=1$ e portanto $\left[\gamma\left(t_{2}\right)\right]^{-1}(1)=\left[\gamma\left(t_{1}\right)\right]^{-1}(1)$. Reciprocamente, suponhamos que $\left[\gamma\left(t_{2}\right)\right]^{-1}(1)=\left[\gamma\left(t_{1}\right)\right]^{-1}(1) \Longleftrightarrow \gamma\left(t_{2}\right) \circ\left[\gamma\left(t_{1}\right)\right]^{-1}(1)=1$. Como $\gamma$ é homomorfismo, então $\gamma\left(t_{1}^{-1} t_{2}\right)=\left[\gamma\left(t_{1}\right)\right]^{-1} \gamma\left(t_{2}\right):=\gamma\left(t_{2}\right) \circ\left[\gamma\left(t_{1}\right)\right]^{-1}$. Logo $\gamma\left(t_{1}^{-1} t_{2}\right)(1)=$ $\gamma\left(t_{2}\right) \circ\left[\gamma\left(t_{1}\right)\right]^{-1}(1)=1$ e portanto $t_{1}^{-1} t_{2} \in D_{n}\left(\mathbb{R P}^{2}\right) \Rightarrow t_{1} D_{n}\left(\mathbb{R P}^{2}\right)=t_{2} D_{n}\left(\mathbb{R P}^{2}\right)$.

Corolário 4.2.3. O índice de $D_{n}\left(\mathbb{R P}^{2}\right)$ em $G_{n}\left(\mathbb{R P}^{2}\right)$ é $n$.

Os elementos $M_{0}=1$ e $M_{i}=\tilde{\sigma}_{i} \ldots \tilde{\sigma}_{1}, i=1, \ldots, n-1$, formam um sistema de representantes das classes laterais a esquerda de $D_{n}\left(\mathbb{R P}^{2}\right)$. De fato, $\gamma\left(M_{0}\right)(1)=1 \mathrm{e}$ $\gamma\left(M_{i}\right)(i+1)=(i, i+1) \ldots(1,2)(i+1)=1 \Rightarrow\left[\gamma\left(M_{i}\right)\right]^{-1}(1)=i+1$. Como $i$ varia de 1 até $n-1$, o conjunto $M:=\left\{M_{0}, M_{1}, \ldots, M_{n-1}\right\}$ tem $n$ elementos e pelo teorema 4.2.2, dois elementos distintos de $M$ não pertencem a mesma classe lateral a esquerda de $D_{n}\left(\mathbb{R} \mathbb{P}^{2}\right)$. Notemos que $M$ é um conjunto de Schreier de $D_{n}\left(\mathbb{R P}^{2}\right)$. Podemos então aplicar o Método de Reidemeister-Schreier para obter o

Teorema 4.2.4. $D_{n}\left(\mathbb{R P}^{2}\right)$ é gerado por $\tilde{\sigma}_{2}, \ldots, \tilde{\sigma}_{n-1}, \tilde{\rho}_{1}, \ldots, \tilde{\rho}_{n}, \tilde{a}_{2}, \ldots, \tilde{a}_{n}$, sendo $\tilde{a}_{i}=\tilde{\sigma}_{1}^{-1} \ldots \tilde{\sigma}_{i-2}^{-1} \tilde{\sigma}_{i-1}^{2} \tilde{\sigma}_{i-2} \ldots \tilde{\sigma}_{1}$, com as seguintes relações: 
(1) $\tilde{\sigma}_{j} \tilde{\sigma}_{k}=\tilde{\sigma}_{k} \tilde{\sigma}_{j},|j-k| \geq 2$

(2) $\tilde{\sigma}_{j} \tilde{\sigma}_{j+1} \tilde{\sigma}_{j}=\tilde{\sigma}_{j+1} \tilde{\sigma}_{j} \tilde{\sigma}_{j+1}$

(3) $\tilde{\sigma}_{j} \tilde{a}_{k} \tilde{\sigma}_{j}^{-1}=\tilde{a}_{k},(k \neq j, j+1)$

(4) $\tilde{\sigma}_{j} \tilde{a}_{j} \tilde{\sigma}_{j}^{-1}=\tilde{a}_{j+1}$

(5) $\tilde{\sigma}_{j} \tilde{a}_{j+1} \tilde{\sigma}_{j}^{-1}=\tilde{a}_{j+1}^{-1} \tilde{a}_{j} \tilde{a}_{j+1}$

(6) $\tilde{\rho}_{1}^{2}=\tilde{a}_{2} \ldots \tilde{a}_{n}$

(7) $\tilde{\rho}_{2} \tilde{a}_{2} \tilde{\rho}_{2}=\tilde{\sigma}_{2} \ldots \tilde{\sigma}_{n-2} \tilde{\sigma}_{n-1}^{2} \tilde{\sigma}_{n-2} \ldots \tilde{\sigma}_{2}$

(8) $\tilde{\sigma}_{j} \tilde{\rho}_{j+1} \tilde{\sigma}_{j}=\tilde{\rho}_{j}$

(9) $\tilde{\rho}_{j} \tilde{\sigma}_{k}=\tilde{\sigma}_{k} \tilde{\rho}_{j}, j \neq k, k+1$

(10) $\tilde{\rho}_{j} \tilde{a}_{k}=\tilde{a}_{k} \tilde{\rho}_{j}, j>k$

(11) $\tilde{\rho}_{j}^{-1} \tilde{a}_{k} \tilde{\rho}_{j}=\tilde{a}_{j} \tilde{a}_{k} \tilde{a}_{j}^{-1}, 1<j<k$

(12) $\tilde{\rho}_{j+1}^{-1} \tilde{\rho}_{j}^{-1} \tilde{\rho}_{j+1} \tilde{\rho}_{j}=\tilde{\sigma}_{j}^{2}, j>1$

(13) $\tilde{\rho}_{j}^{-1} \tilde{\rho}_{1} \tilde{\rho}_{j}=\tilde{\rho}_{1} \tilde{a}_{j}^{-1}, j>1$

(14) $\tilde{\rho}_{j} \tilde{a}_{j} \tilde{\rho}_{j}^{-1}=\tilde{\rho}_{1}^{-1} \tilde{a}_{2} \ldots \tilde{a}_{j-1} \tilde{a}_{j}^{-1} \tilde{a}_{j-1}^{-1} \ldots \tilde{a}_{2}^{-1} \tilde{\rho}_{1}$

Omitimos a prova do teorema 4.2.4, por se tratar de aplicação direta do Método de Reidemeister-Schreier e por conter apenas extensos cálculos.

Seja $A_{n}\left(\mathbb{R} \mathbb{P}^{2}\right)$ o subgrupo de $D_{n}\left(\mathbb{R} \mathbb{P}^{2}\right)$ gerado por $\tilde{\rho}_{1}, \tilde{a}_{2}, \ldots, \tilde{a}_{n}$.

Teorema 4.2.5. $A_{n}\left(\mathbb{R P}^{2}\right)$ é um subgrupo normal de $D_{n}\left(\mathbb{R P}^{2}\right)$.

Prova: Os únicos geradores de $D_{n}\left(\mathbb{R P}^{2}\right)$ que não são geradores de $A_{n}\left(\mathbb{R P}^{2}\right)$ são $\tilde{\sigma}_{2}, \ldots, \tilde{\sigma}_{n-1}, \tilde{\rho}_{2}, \ldots, \tilde{\rho}_{n}$. Portanto, para demonstrarmos o teorema, basta mostrarmos que os geradores de $A_{n}\left(\mathbb{R P}^{2}\right)$ conjugados por esses elementos pertencem a $A_{n}\left(\mathbb{R P}^{2}\right)$. Mas esses cálculos seguem diretamente das relações que obtivemos para $D_{n}\left(\mathbb{R P}^{2}\right)$ e por isso os omitimos.

Teorema 4.2.6. Se $n>1$, então $D_{n}\left(\mathbb{R P}^{2}\right) / A_{n}\left(\mathbb{R P}^{2}\right)$ é isomorfo a $G_{n-1}\left(\mathbb{R} \mathbb{P}^{2}\right)$.

Prova: Uma apresentação de $D_{n}\left(\mathbb{R P}^{2}\right) / A_{n}\left(\mathbb{R P}^{2}\right)$ é obtida da apresentação de $D_{n}\left(\mathbb{R P}^{2}\right)$ adicionando as relações $\tilde{\rho}_{1}=1, \tilde{a}_{2}=1, \tilde{a}_{3}=1, \ldots, \tilde{a}_{n}=1$. A adição destas relações transforma as relações $(3),(4),(5),(6),(10),(11),(13),(14)$ em relações triviais e altera a relação (7) para $\tilde{\rho}_{2}^{2}=\tilde{\sigma}_{2} \tilde{\sigma}_{3} \tilde{\sigma}_{n-2} \tilde{\sigma}_{n-1}^{2} \tilde{\sigma}_{n-2} \ldots \tilde{\sigma}_{3} \tilde{\sigma}_{2}$. Portanto, $D_{n}\left(\mathbb{R} \mathbb{P}^{2}\right) / A_{n}\left(\mathbb{R} \mathbb{P}^{2}\right)$ é gerado por $\tilde{\sigma}_{2}, \tilde{\sigma}_{3}, \ldots, \tilde{\sigma}_{n-1}, \tilde{\rho}_{2}, \ldots, \tilde{\rho}_{n}$ sujeito as seguintes relações:

(1) $\tilde{\sigma}_{j} \tilde{\sigma}_{k}=\tilde{\sigma}_{k} \tilde{\sigma}_{j},|j-k| \geq 2$

(2) $\tilde{\sigma}_{j} \tilde{\sigma}_{j+1} \tilde{\sigma}_{j}=\tilde{\sigma}_{j+1} \tilde{\sigma}_{j} \tilde{\sigma}_{j+1}$

(9) $\tilde{\rho}_{j} \tilde{\sigma}_{k}=\tilde{\sigma}_{k} \tilde{\rho}_{j}, j \neq k, k+1$

(8) $\tilde{\sigma}_{j} \tilde{\rho}_{j+1} \tilde{\sigma}_{j}=\tilde{\rho}_{j}$ 
(12) $\tilde{\rho}_{j+1}^{-1} \tilde{\rho}_{j}^{-1} \tilde{\rho}_{j+1} \tilde{\rho}_{j}=\tilde{\sigma}_{j}^{2}, j>1$

(7) $\tilde{\rho}_{2}^{2}=\tilde{\sigma}_{2} \tilde{\sigma}_{3} \tilde{\sigma}_{n-2} \tilde{\sigma}_{n-1}^{2} \tilde{\sigma}_{n-2} \ldots \tilde{\sigma}_{3} \tilde{\sigma}_{2}$.

A menos de renomeação de índices, esta é a apresentação de $G_{n-1}\left(\mathbb{R P}^{2}\right)$.

Pelo resultado anterior, vamos considerar $G_{n-1}\left(\mathbb{R P}^{2}\right)=D_{n}\left(\mathbb{R P}^{2}\right) / A_{n}\left(\mathbb{R P}^{2}\right)$.

Teorema 4.2.7. Sejam $n>1, i: A_{n}\left(\mathbb{R P}^{2}\right) \rightarrow D_{n}\left(\mathbb{R P}^{2}\right)$ a inclusão e $j: D_{n}\left(\mathbb{R P}^{2}\right) \rightarrow$ $G_{n-1}\left(\mathbb{R P}^{2}\right)$ a projeção natural. Então a sequência exata

$$
1 \longrightarrow A_{n}\left(\mathbb{R P}^{2}\right) \stackrel{i}{\longrightarrow} D_{n}\left(\mathbb{R P}^{2}\right) \stackrel{j}{\longrightarrow} G_{n-1}\left(\mathbb{R P}^{2}\right) \longrightarrow 1
$$

induz uma sequência exata

$$
1 \longrightarrow A_{n}\left(\mathbb{R P}^{2}\right) \stackrel{i}{\longrightarrow} K_{n}\left(\mathbb{R P}^{2}\right) \stackrel{j}{\longrightarrow} K_{n-1}\left(\mathbb{R P}^{2}\right) \longrightarrow 1
$$

chamada sequência exata fundamental de $G_{n}\left(\mathbb{R P}^{2}\right)$.

Prova: Podemos identificar $S_{n-1}$ como o subgrupo de $S_{n}$ que deixa o número 1 fixo. Então, o seguinte diagrama é comutativo

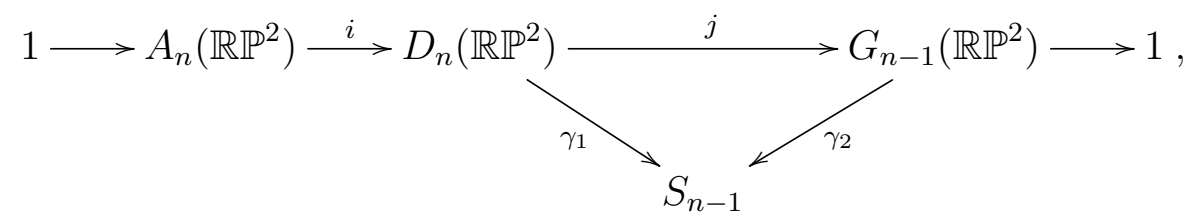

sendo $\gamma_{1}$ a restrição do homomorfismo permutação $\gamma: G_{n}\left(\mathbb{R P}^{2}\right) \rightarrow S_{n}$ e $\gamma_{2}$ é o homorfismo permutação de $G_{n-1}\left(\mathbb{R P}^{2}\right)$, a menos de renomeação dos índices. Claramente, a seguinte sequência é exata:

$$
1 \longrightarrow A_{n}\left(\mathbb{R P}^{2}\right) \stackrel{i}{\longrightarrow} \operatorname{ker} \gamma_{1} \stackrel{j}{\longrightarrow} \operatorname{ker} \gamma_{2} \longrightarrow 1
$$

Mas ker $\gamma_{1}=K_{n}\left(\mathbb{R P}^{2}\right)$ e ker $\gamma_{2} \approx K_{n-1}\left(\mathbb{R P}^{2}\right)$. Segue o resultado.

Teorema 4.2.8. $G_{2}\left(\mathbb{R P}^{2}\right)$ é isomorfo ao grupo dicíclico de ordem 16 .

Prova: Por definição, $G_{2}\left(\mathbb{R} \mathbb{P}^{2}\right)$ é gerado por $\tilde{\rho}_{1}, \tilde{\rho}_{2}$ e $\tilde{\sigma}_{1}$, com as seguintes relações:
(iv) $\tilde{\rho}_{1}=\tilde{\sigma}_{1} \tilde{\rho}_{2} \tilde{\sigma}_{1}$
(v) $\tilde{\rho}_{2}^{-1} \tilde{\rho}_{1}^{-1} \tilde{\rho}_{2} \tilde{\rho}_{1}=\tilde{\sigma}_{1}^{2}$
(vi) $\tilde{\rho}_{1}^{2}=\tilde{\sigma}_{1}^{2}$

Da relação (iv) segue que $\tilde{\rho}_{2}=\tilde{\sigma}_{1}^{-1} \tilde{\rho}_{1} \tilde{\sigma}_{1}^{-1}$ e vamos usar esta igualdade como definição de $\tilde{\rho}_{2}$. Logo, $G_{2}\left(\mathbb{R} \mathbb{P}^{2}\right)=\left\langle\tilde{\rho}_{1}, \tilde{\sigma}_{1} \mid \tilde{\sigma}_{1} \tilde{\rho}_{1}^{-1} \tilde{\sigma}_{1} \tilde{\rho}_{1}^{-1} \tilde{\sigma}_{1}^{-1} \tilde{\rho}_{1} \tilde{\sigma}_{1}^{-1} \tilde{\rho}_{1}=\tilde{\rho}_{1}^{2}=\tilde{\sigma}_{1}^{2}\right\rangle$. Notemos que 
(vi) $\tilde{\rho}_{1}^{2}=\tilde{\sigma}_{1}^{2} \Longleftrightarrow \tilde{\sigma}_{1}^{-1} \tilde{\rho}_{1}=\tilde{\sigma}_{1} \tilde{\rho}_{1}^{-1}$.

Portanto, $\tilde{\sigma}_{1} \tilde{\rho}_{1}^{-1} \tilde{\sigma}_{1} \tilde{\rho}_{1}^{-1} \tilde{\sigma}_{1}^{-1} \tilde{\rho}_{1} \tilde{\sigma}_{1}^{-1} \tilde{\rho}_{1}=\tilde{\sigma}_{1} \tilde{\rho}_{1}^{-1} \tilde{\sigma}_{1} \tilde{\rho}_{1}^{-1}\left(\tilde{\sigma}_{1}^{-1} \tilde{\rho}_{1}\right)\left(\tilde{\sigma}_{1}^{-1} \tilde{\rho}_{1}\right) \stackrel{(*)}{=} \tilde{\sigma}_{1} \tilde{\rho}_{1}^{-1} \tilde{\sigma}_{1} \tilde{\rho}_{1}^{-1} \tilde{\sigma}_{1} \tilde{\rho}_{1}^{-1} \tilde{\sigma}_{1} \tilde{\rho}_{1}^{-1}$ $=\left(\tilde{\sigma}_{1} \tilde{\rho}_{1}^{-1}\right)^{4}$. Assim, concluímos que $G_{2}\left(\mathbb{R} \mathbb{P}^{2}\right)=\left\langle\tilde{\rho}_{1}, \tilde{\sigma}_{1} \mid\left(\tilde{\sigma}_{1} \tilde{\rho}_{1}^{-1}\right)^{4}=\tilde{\rho}_{1}^{2}=\tilde{\sigma}_{1}^{2}\right\rangle$, a qual é uma apresentação para $D i c_{16}$, conforme foi provado na seção 1.5 .

Teorema 4.2.9. $K_{2}\left(\mathbb{R P}^{2}\right)$ é isomorfo ao grupo dos quatérnios.

Prova: Como $S_{2}$ tem ordem dois e pelo teorema $4.2 .8 G_{2}\left(\mathbb{R P}^{2}\right)$ tem ordem 16, então pelo teorema de Lagrange, $K_{2}\left(\mathbb{R P}^{2}\right)$ tem ordem 8 . Agora, por definição, $\gamma: G_{2}\left(\mathbb{R}^{2}\right) \rightarrow$ $S_{2}$, mapeia $\tilde{\sigma}_{1}$ na transposição $(1,2)$ e mapeia os elementos $\tilde{\rho}_{1}$ e $\tilde{\rho}_{2}$ na permutação identidade. Se adicionarmos as relações $\tilde{\rho}_{1}=\tilde{\rho}_{2}=1 \mathrm{em} G_{2}\left(\mathbb{R}^{2}\right)$, obteremos o grupo $\left\langle\tilde{\sigma}_{1} \mid \tilde{\sigma}_{1}^{2}=1\right\rangle$, que é uma apresentação de $S_{2}$. Portanto, o grupo $K_{2}\left(\mathbb{R}^{2}\right)$, que é o núcleo de $\gamma$, é consequência destas duas relações adicionais, isto é, $K_{2}\left(\mathbb{R P}^{2}\right)$ é o menor subgrupo normal de $G_{2}\left(\mathbb{R}^{2}\right)$ que contém $\tilde{\rho}_{1}$ e $\tilde{\rho}_{2}$. Mas o subgrupo gerado por $\tilde{\rho}_{1}$ e $\tilde{\rho}_{2}$ é normal em $G_{2}\left(\mathbb{R}^{2}\right)$. Para vermos isto, basta mostrarmos que $\tilde{\rho}_{1}$ e $\tilde{\rho}_{2}$ conjugados por $\tilde{\sigma}_{1}$ pertencem ao subgrupo em questão. Mas isto é verdade, pois notemos que

$$
\text { (iv) } \Longleftrightarrow \sigma_{1}^{-1} \tilde{\rho}_{1}=\tilde{\rho}_{2} \tilde{\sigma}_{1}
$$

e portanto

$$
\tilde{\sigma}_{1}^{-1} \tilde{\rho}_{1} \tilde{\sigma}_{1} \stackrel{(* *)}{=} \tilde{\rho}_{2} \tilde{\sigma}_{1}^{2} \stackrel{(\mathrm{vi})}{=} \tilde{\rho}_{2} \tilde{\rho}_{1}^{2} \quad \tilde{\sigma}_{1}^{-1} \tilde{\rho}_{2} \tilde{\sigma}_{1} \stackrel{(* *)}{=} \tilde{\sigma}_{1}^{-1} \tilde{\sigma}_{1}^{-1} \tilde{\rho}_{1} \stackrel{(\mathrm{vi})}{=} \tilde{\rho}_{1}
$$

Vamos extrair algumas relações entre estes geradores. Pelo teorema 4.2.8, $\tilde{\sigma}_{1}^{2}=$ $\left(\tilde{\sigma}_{1} \tilde{\rho}_{1}^{-1}\right)^{4}$. Usando $(*)$ também do teorema 4.2 .8 , temos que $\tilde{\sigma}_{1}^{2}=\left(\tilde{\sigma}_{1} \tilde{\rho}_{1}^{-1}\right)^{4} \stackrel{(*)}{=}\left(\tilde{\rho}_{1} \tilde{\sigma}_{1}^{-1}\right)^{4}$ $=\left(\tilde{\sigma}_{1} \tilde{\rho}_{1}^{-1}\right)^{-4}=\tilde{\sigma}_{1}^{-2} \Rightarrow \tilde{\sigma}_{1}^{4}=1$. Por (vi), obtemos que $\tilde{\rho}_{1}^{4}=1 \Longleftrightarrow \tilde{\rho}_{1}^{2}=\tilde{\rho}_{1}^{-2}$. Agora, $\tilde{\rho}_{2}^{2} \stackrel{(\mathrm{iv})}{=}\left(\tilde{\sigma}_{1}^{-1} \tilde{\rho}_{1}^{-1} \tilde{\sigma}_{1}^{-1}\right)^{2}=\tilde{\sigma}_{1}^{-1} \tilde{\rho}_{1} \tilde{\sigma}_{1}^{-2} \tilde{\rho}_{1} \tilde{\sigma}_{1}^{-1} \stackrel{(\mathrm{vi})}{=} \tilde{\sigma}_{1}^{-1} \tilde{\rho}_{1} \tilde{\rho}_{1}^{-2} \tilde{\rho}_{1} \tilde{\sigma}_{1}^{-1}=\tilde{\sigma}_{1}^{-2} \stackrel{(\mathrm{vi})}{=} \tilde{\rho}_{1}^{-2}=\tilde{\rho}_{1}^{2}$. Esta igualde implica que

$\tilde{\rho}_{2} \tilde{\rho}_{1}=\tilde{\rho}_{2}^{-1} \tilde{\rho}_{1}^{-1}$.
$\left.\operatorname{Logo}, \tilde{\rho}_{1}^{2}=\tilde{\sigma}_{1}^{2} \stackrel{(\mathrm{v})}{=} \tilde{\rho}_{2}^{-1} \tilde{\rho}_{1}^{-1} \tilde{\rho}_{2} \tilde{\rho}_{1} \stackrel{(* * *)}{=}{ }_{\left(\tilde{\rho}_{2}\right.} \tilde{\rho}_{1}\right)^{2}$. Portanto, fica claro que $\left(\tilde{\rho}_{2} \tilde{\rho}_{1}\right)^{2}=\tilde{\rho}_{2}^{2}=\tilde{\rho}_{1}^{2}$. Se $K_{2}\left(\mathbb{R P}^{2}\right)$ não fosse igual a $\left\langle\tilde{\rho}_{1}, \tilde{\rho}_{2} \mid\left(\tilde{\rho}_{2} \tilde{\rho}_{1}\right)^{2}=\tilde{\rho}_{2}^{2}=\tilde{\rho}_{1}^{2}\right\rangle$, que é uma apresentação para o grupo dos quatérnios de acordo com a seção 1.5, então existiria pelo menos mais uma relação não trivial entre $\tilde{\rho}_{1}$ e $\tilde{\rho}_{2}$ que não provém desta que obtivemos. Mas então, $K_{2}\left(\mathbb{R P}^{2}\right)$ teria ordem menor que 8 , o que é um absurdo. Portanto, $K_{2}\left(\mathbb{R P}^{2}\right)$ é isomorfo ao grupo dos quatérnios. 
Corolário 4.2.10. Em $G_{2}\left(\mathbb{R P} \mathbb{P}^{2}\right),\left(\tilde{\rho}_{1} \tilde{\rho}_{2}\right)^{2}=\tilde{\sigma}_{1}^{2}$ e este elemento tem ordem 2.

Prova: No teorema anterior obtivemos a relação $\left(\tilde{\rho}_{2} \tilde{\rho}_{1}\right)^{2}=\tilde{\rho}_{1}^{2}$ a qual ocorre se, e somente se, $\tilde{\rho}_{2} \tilde{\rho}_{1} \tilde{\rho}_{2}=\tilde{\rho}_{1}$. Logo, $\left(\tilde{\rho}_{1} \tilde{\rho}_{2}\right)^{2}=\tilde{\rho}_{1}\left(\tilde{\rho}_{2} \tilde{\rho}_{1} \tilde{\rho}_{2}\right)=\tilde{\rho}_{1}^{2}$. Como já mostramos, $\tilde{\rho}_{1}^{2}=\tilde{\sigma}_{1}^{2}$. Segue a igualdade. Também mostramos no teorema anterior que $\tilde{\sigma}_{1}^{4}=1$. Logo, para mostrarmos que $\left(\tilde{\rho}_{1} \tilde{\rho}_{2}\right)^{2}$ tem ordem 2 , é suficiente mostrarmos que $\left(\tilde{\rho}_{1} \tilde{\rho}_{2}\right)^{2} \neq 1$. Mas se valesse a igualdade, então, pelos cálculos que fizemos no teorema anterior, teríamos obtido que $K_{2}\left(\mathbb{R P}^{2}\right)=\left\langle\tilde{\rho}_{1}, \tilde{\rho}_{2} \mid \tilde{\rho}_{1}^{2}=\tilde{\rho}_{2}^{2}=1\right\rangle$, a qual é uma apresentação de $\mathbb{Z}_{2} \times \mathbb{Z}_{2}$, que tem ordem 4 e isto contraria o resultado anterior.

Teorema 4.2.11. $A_{2}\left(\mathbb{R P}^{2}\right)$ é cíclico de ordem 4.

Prova: Consideremos a sequência exata fundamental

$$
1 \longrightarrow A_{2}\left(\mathbb{R P}^{2}\right) \stackrel{i}{\longrightarrow} K_{2}\left(\mathbb{R P}^{2}\right) \stackrel{j}{\longrightarrow} K_{1}\left(\mathbb{R P}^{2}\right) \longrightarrow 1
$$

Notemos que $K_{1}\left(\mathbb{R P}^{2}\right)=G_{1}\left(\mathbb{R P}^{2}\right)=\left\langle\tilde{\rho}_{2} \mid \tilde{\rho}_{2}^{2}=1\right\rangle$, que é cíclico de ordem 2. Portanto, como $K_{2}$ tem ordem 8 , pelo teorema anterior, então $A_{2}\left(\mathbb{R P}^{2}\right)$ tem ordem 4 . Mas desde que os geradores $\tilde{\rho}_{1}$ e $\tilde{a}_{2}$ de $A_{2}\left(\mathbb{R P}^{2}\right)$ satisfazem $\tilde{a}_{2}=\tilde{\sigma}_{1}^{2}=\tilde{\rho}_{1}^{2}, \tilde{\rho}_{1}^{4}=1$, e $\tilde{\rho}_{1}^{2} \neq 1$, então $A_{2}\left(\mathbb{R P}^{2}\right)=\left\langle\tilde{\rho}_{1} \mid \tilde{\rho}_{1}^{4}=1\right\rangle$.

Teorema 4.2.12. Se $n>2$, então $A_{n}\left(\mathbb{R P}^{2}\right)$ é um grupo livre de rank $n-1$ e neste caso $A_{n}\left(\mathbb{R} \mathbb{P}^{2}\right)=\left\langle\tilde{\rho}_{1}, \tilde{a}_{2}, \tilde{a}_{3} \ldots, \tilde{a}_{n} \mid \tilde{\rho}_{1}^{2}=\tilde{a}_{2} \tilde{a}_{3} \ldots \tilde{a}_{n}\right\rangle$.

Prova: Consideremos $U_{n}=\left\langle v, u_{2}, u_{3}, \ldots, u_{n} \mid v^{2}=u_{2} u_{3} \ldots u_{n}\right\rangle$ o grupo livre de rank $n-1$. De fato $U_{n}$ é livre, pois $u_{n}=u_{n-1}^{-1} \ldots u_{3}^{-1} u_{2}^{-1} v^{2}$, ou seja, podemos retirar $u_{n}$ do conjunto de geradores e não existe relações entre os demais geradores. Vamos exibir uma representação de $D_{n}\left(\mathbb{R P}^{2}\right)$ em $A u t\left(U_{n}\right)$, tal que $A_{n}\left(\mathbb{R P}^{2}\right)$ está em correspondência biunívoca com $\mathcal{I}_{U_{n}}$, sendo $\mathcal{I}_{U_{n}}$ o subgrupo dos automorfismos internos de $U_{n}$. Como o rank de $U_{n}$ é maior que 1 , pelo lema 1.6.1, seguirá que $A_{n}\left(\mathbb{R P}^{2}\right)$ é um grupo livre de rank $n-1$. Para cada gerador de $D_{n}\left(\mathbb{R P}^{2}\right)$, denotaremos pelo mesmo símbolo o automorfismo associado em $A u t\left(U_{n}\right)$. Definimos:

$$
\begin{aligned}
& \tilde{\sigma}_{i}: u_{j} \mapsto u_{j}, \text { se } j \neq i, i+1, u_{i} \mapsto u_{i+1}, u_{i+1} \mapsto u_{i+1}^{-1} u_{i}^{-1} u_{i+1}, v \mapsto v \\
& \tilde{a}_{i}: u_{j} \mapsto u_{i} u_{j} u_{i}^{-1}, v \mapsto u_{i} v u_{i}^{-1} \\
& \tilde{\rho}_{1}: u_{j} \mapsto v u_{j} v^{-1}, v \mapsto v \text { para } i>1 \\
& \tilde{\rho}_{i}: u_{j} \mapsto w^{-1} u_{j} w, \text { se } j>i, u_{j} \mapsto u_{j}, \text { se } j<i, u_{i} \mapsto w, v \mapsto v w
\end{aligned}
$$

sendo $w=v^{-1} u_{2} \ldots u_{i-1} u_{i} u_{i-1}^{-1} \ldots u_{2}^{-1} v$. Estes automorfismos satisfazem as relações 
(1)...(14) e por argumentos puramente combinatórios mostra-se que essa associação é injetora. Notemos que os automorfismos $\tilde{a}_{2}, \ldots, \tilde{a}_{n}, \tilde{\rho}_{1}$ geram $\mathcal{I}_{U_{n}}$. Como $\tilde{\rho}_{1}^{2} \stackrel{(6)}{=}$ $\tilde{a}_{2} \tilde{a}_{3} \ldots \tilde{a}_{n}$, e $A_{n}\left(\mathbb{R P}^{2}\right)$ é livre de rank $n-1$, segue que $A_{n}\left(\mathbb{R P}^{2}\right)=\left\langle\tilde{\rho}_{1}, \tilde{a}_{2}, \tilde{a}_{3} \ldots, a_{n} \mid \tilde{\rho}_{1}^{2}=\tilde{a}_{2} \tilde{a}_{3} \ldots a_{n}\right\rangle$.

Corolário 4.2.13. $G_{n}\left(\mathbb{R P}^{2}\right)$ é infinito para $n>2$.

\subsection{Propriedades de $B_{n}\left(\mathbb{R} \mathbb{P}^{2}\right)$}

Definição 4.3.1. O espaço das n-uplas de pontos da esfera $\mathbb{S}^{2}$ que são distintos $e$ não antipodais, com a topologia induzida de $\underbrace{\mathbb{S}^{2} \times \ldots \times \mathbb{S}^{2}}_{n-v e z e s}$, é chamado de espaço de configuração de $n$ pontos ordenados e não anti podais de $\mathbb{S}^{2}$ e denotado por $\mathcal{A}_{n}\left(\mathbb{S}^{2}\right)$, isto é, $\mathcal{A}_{n}\left(\mathbb{S}^{2}\right)=\left\{\left(x_{1}, \ldots, x_{n}\right) \in \prod_{1}^{n} \mathbb{S}^{2} \mid x_{i} \neq \pm x_{j}, i \neq j\right\}$.

No próximo resultado, consideraremos $\mathbb{R} \mathbb{P}^{2}$ como $\mathbb{S}^{2}$ passada a quociente pela relação que identifica pontos antípodas.

Teorema 4.3.2. A aplicação $p: \mathcal{A}_{n}\left(\mathbb{S}^{2}\right) \rightarrow F_{n}\left(\mathbb{R P}^{2}\right)$, definida por $p\left(x_{1}, \ldots, x_{n}\right)=$ $\left(\left[x_{1}\right], \ldots,\left[x_{n}\right]\right)$, é um recobrimento a $2^{n}$ folhas.

Prova: Seja $\left(\left[x_{1}\right], \ldots,\left[x_{n}\right]\right) \in F_{n}\left(\mathbb{R P}^{2}\right)$. Consideremos a aplicação $\tilde{p}: \mathbb{S}^{2} \rightarrow \mathbb{R} \mathbb{P}^{2}$, dada por $\tilde{p}(x)=[x]$, a qual é um recobrimento a duas folhas. Logo, para todo $i=1, \ldots, n$, existem abertos $U_{i} \subset \mathbb{S}^{2}$ e $V_{i} \subset \mathbb{R P}^{2}$, tais que $x_{i} \in U_{i},\left[x_{i}\right] \in V_{i}, \tilde{p}^{-1}\left(V_{i}\right)=U_{i} \cup-U_{i}$ e $\left.\tilde{p}\right|_{ \pm U_{i}}: \pm U_{i} \rightarrow V_{i}$ são homeomorfismos. Como $\mathbb{S}^{2}$ e $\mathbb{R} \mathbb{P}^{2}$ são espaços de Hausdorff, podemos considerar tais abertos de modo que $U_{i} \cap \pm U_{j}=\emptyset$ e $V_{i} \cap V_{j}=\emptyset$, sempre que $i \neq j$. Denotando $U_{i}^{1}=U_{i}, U_{i}^{2}=-U_{i}, T_{i_{1}, \ldots, i_{n}}=U_{1}^{i_{1}} \times \ldots \times U_{n}^{i_{n}}, i_{1}, \ldots, i_{n} \in\{1,2\}$, $V=V_{1}, \ldots, V_{n}$, então $V$ é um aberto de $F_{0, n}\left(\mathbb{R P}^{2}\right)$ que contém $\left(\left[x_{1}\right], \ldots,\left[x_{n}\right]\right)$, os conjuntos $T_{i_{1}, \ldots, i_{n}}$ são abertos disjuntos de $\mathcal{A}_{n}\left(\mathbb{S}^{2}\right)$, com $i_{j} \in\{1,2\}$ e notando que $p\left(x_{1}, \ldots, x_{n}\right)=\left(\tilde{p}\left(x_{1}\right), \ldots, \tilde{p}\left(x_{n}\right)\right), p^{-1}(V)=\bigcup T_{i_{1}, \ldots, i_{n}}$ e $p$ restrita a cada aberto desta união disjunta é um homeomorfismo com $V$. Segue o resultado.

Lema 4.3.3. $\mathcal{A}_{2}\left(\mathbb{S}^{2}\right)$ tem o mesmo tipo de homotopia que $V_{3,2}$, sendo $V_{3,2}=\left\{\left(v_{1}, v_{2}\right) \in\right.$ $\left.\mathbb{R}^{3} \times \mathbb{R}^{3} \mid<v_{i}, v_{j}>=\delta_{i, j}\right\}$ uma variedade de Stiefel.

Observação 4.3.4. O símbolo $<$, $>$ denota o produto interno entre dois vetores de $\mathbb{R}^{3}$. O símbolo $\delta_{i, j}$ denota o delta de Kronecker. Para mais informações sobre variedades de Stiefel, citamos [20] e [21].

Prova: Consideremos $\mathcal{A}_{2}\left(\mathbb{S}^{2}\right)=\left\{(a, b) \mid a, b \in \mathbb{S}^{2}, a \neq \pm b\right\}$ e $V_{3,2}=\{(x, y) \mid x, y \in$ $\left.\mathbb{R}^{3},\|x\|=\|y\|=1,<x, y>=0\right\}$ e definimos 


$$
\begin{gathered}
f: V_{3,2} \rightarrow \mathcal{A}_{2}\left(\mathbb{S}^{2}\right) \\
(x, y) \mapsto(x, y)
\end{gathered}
$$$$
g: \mathcal{A}_{2}\left(\mathbb{S}^{2}\right) \rightarrow V_{3,2}
$$$$
(a, b) \mapsto\left(a, \frac{a \wedge b}{\|a \wedge b\|}\right)
$$

$\mathrm{O}$ símbolo $\wedge$ denota o produto vetorial entre dois vetores

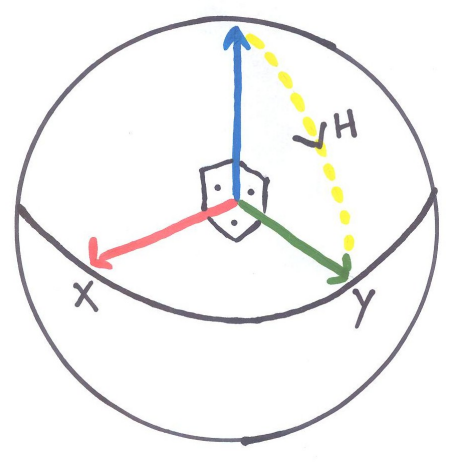
de $\mathbb{R}^{3}$. Uma definição de produto vetorial e algumas de suas propriedades estão feitas, de modo suscinto, em [18]. Notemos que $g$ está bem definida. De fato, segue da definição de produto vetorial que $\langle a, a \wedge b\rangle=0$ e como $a$ não é um vetor múltiplo de $b$, então $a \wedge b \neq 0$. Como a norma e produto vetorial de dois vetores dependem continuamente das coordenadas dos dois vetores, segue que $g$ é contínua. Agora definimos

Figura 4.8: homotopia $H$

$$
\begin{aligned}
& H: V_{3,2} \times[0,1] \rightarrow V_{3,2} \\
& J: \mathcal{A}_{2}\left(\mathbb{S}^{2}\right) \times[0,1] \rightarrow \mathcal{A}_{2}\left(\mathbb{S}^{2}\right) \\
& ((x, y), t) \mapsto\left(x, \frac{(1-t) x \wedge y+t y}{\|(1-t) x \wedge y+t y\|}\right) \quad(a, b) \mapsto\left(a, \frac{(1-t) a \wedge b+\|a \wedge b\| t b}{\|(1-t) a \wedge b+\| a \wedge b \| t b} \|\right.
\end{aligned}
$$

Para mostrarmos que $H$ e $J$ estão bem definidas e são contínuas, basta usarmos as propriedades de norma, produto interno e produto vetorial de $\mathbb{R}^{3}$. A figura 4.8 nos dá uma ideia da homotopia $H$. A homotopia $J$ é semelhante. Agora notemos que

$$
\begin{aligned}
& H((x, y), 0)=\left(x, \frac{x \wedge y}{\|x \wedge y\|}\right)=g \circ f(x, y) \quad J((a, b), 0)=\left(a, \frac{a \wedge b}{\|a \wedge b\|}\right)=f \circ g(a, b) \\
& H((x, y), 1)=\left(x, \frac{y}{\|y\|}\right)=(x, y)=I d_{V_{3,2}} \quad J((a, b), 1)=\left(a, \frac{b}{\|b\|}\right)=(a, b)=I_{\mathcal{A}_{2}\left(S^{2}\right)}
\end{aligned}
$$

Com isto concluímos que $\mathcal{A}_{2}\left(\mathbb{S}^{2}\right)$ tem o mesmo tipo de homotopia que $V_{3,2}$.

Lema 4.3.5. $\pi_{2}\left(F_{n-1,1}\left(\mathbb{R P}^{2}\right)\right)=1$ para todo $n \geq 2$

Prova: Notemos que $F_{n-1,1}\left(\mathbb{R P}^{2}\right)$ é o espaço projetivo menos $n-1$ pontos, a qual tem o mesmo tipo de homotopia que um bouquet de $n-1 \mathbb{S}^{1}$, que é um grafo. A figura 4.9 nos dá uma ideia desta afirmação. O resultado segue dos fatos que o recobrimento universal de um grafo é uma árvore, toda árvore é contrátil e uma aplicação de recobrimento induz isomorfismo nos grupos de homotopia de dimensão maior que 1. 

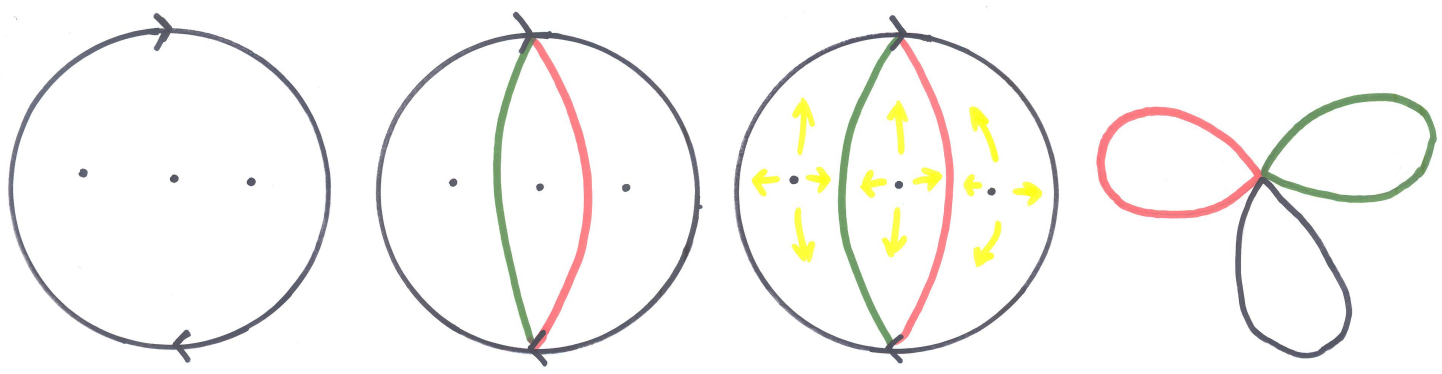

Figura 4.9: deformação de $\mathbb{R P}^{2}$ num bouquet de $\mathbb{S}^{1}$

Lema 4.3.6. Se $n \geq 2$, então $\pi_{2}\left(F_{0, n}\left(\mathbb{R P}^{2}\right)\right)=1$.

Prova: A prova é feita por indução em $n$. Em [22] encontramos uma prova que $\pi_{2}\left(V_{3,2}\right)=1$. Segue do teorema 4.3 .2 e do lema 4.3 .3 que $\pi_{2}\left(F_{0,2}\left(\mathbb{R P}^{2}\right)\right)=1$. Agora suponhamos que $\pi_{2}\left(F_{0, n-1}\left(\mathbb{R P}^{2}\right)\right)=1$. O teorema de Fadell-Neuwirth (teorema 1.2.2) fornece a seguinte sequência exata:

$$
\ldots \longrightarrow \pi_{2}\left(F_{n-1,1}\left(\mathbb{R P}^{2}\right)\right) \longrightarrow \pi_{2}\left(F_{0, n}\left(\mathbb{R P}^{2}\right)\right) \longrightarrow \pi_{2}\left(F_{0, n-1}\left(\mathbb{R P}^{2}\right)\right) \longrightarrow \ldots
$$

Aplicando o lema 4.3.5 e a hipótese de indução na sequência acima, concluímos que $\pi_{2}\left(F_{0, n}\left(\mathbb{R P}^{2}\right)\right)=1$.

Seja $n \geq 3$ e consideremos a fibração $\pi: F_{0, n}\left(\mathbb{R P}^{2}\right) \rightarrow F_{0, n-1}\left(\mathbb{R P}^{2}\right)$ definida no teorema de Fadell-Neuwirth (teorema 1.2.2), cuja fibra típica é $F_{n-1,1}\left(\mathbb{R P}^{2}\right)$. Notemos que $\pi_{0}\left(F_{n-1,1}\left(\mathbb{R P}^{2}\right)\right)=1$, já que $\mathbb{R P}^{2}$ menos uma quantidade finita de pontos é um espaço conexo por caminhos. Logo, a sequência exata de homotopia da fibração $\pi$, junto com o lema 4.3.6, nos fornece a seguinte sequência exata curta:

$$
1 \longrightarrow \pi_{1}\left(F_{n-1,1}\left(\mathbb{R P}^{2}\right)\right) \stackrel{i_{*}}{\longrightarrow} \pi_{1}\left(F_{0, n}\left(\mathbb{R P}^{2}\right)\right) \stackrel{\pi_{*}}{\longrightarrow} \pi_{1}\left(F_{0, n-1}\left(\mathbb{R P}^{2}\right)\right) \longrightarrow 1
$$

sendo $i_{*}$ a induzida da inclusão.

Mas, por definição $P_{n}\left(\mathbb{R P}^{2}\right)=\pi_{1}\left(F_{0, n}\left(\mathbb{R P}^{2}\right)\right)$ e assim temos a seguinte

Definição 4.3.7. Para $n \geq 3$, a sequência exata curta

$$
1 \longrightarrow \pi_{1}\left(F_{n-1,1}\left(\mathbb{R P}^{2}\right)\right) \stackrel{i_{*}}{\longrightarrow} P_{n}\left(\mathbb{R P}^{2}\right) \stackrel{\pi_{*}}{\longrightarrow} P_{n-1}\left(\mathbb{R P}^{2}\right) \longrightarrow 1
$$

é chamada de sequência exata curta de Fadell-Neuwirth do $\mathbb{R P}^{2}$. 
Geometricamente, o homomorfismo $\pi_{*}$ corresponde a retirar a primeira corda de uma trança.

Vamos dar uma interpretação geométrica do homomorfismo $i_{*}$. Mas antes temos o

Teorema 4.3.8. Se $n \geq 2$, então $\pi_{1}\left(F_{n-1,1}\left(\mathbb{R P}^{2}\right)\right)$ é um grupo livre de rank $n-1$, cuja apresentação é $\left\langle\rho_{1}, a_{1,2}, \ldots, a_{1, n} \mid \rho_{1}^{2}=a_{1,2} \ldots a_{1, n}\right\rangle$.

Prova: Consideremos os abertos $U$ e $V$ de $\mathbb{R P}^{2}$, conforme as figuras 4.10 e 4.11 . Notemos que o aberto $V$ tem o mesmo tipo que homotopia que $\mathbb{S}^{1}$. Logo $\pi_{1}(V)$ é cíclico infinito. Na figura 4.11 também está desenhado um gerador de $\pi_{1}(V)$, que chamamos de $\rho_{1}$. Agora, é fácil ver que $\pi_{1}(U)$ é livre de rank $n-1$. Sejam $a_{1,2}, \ldots, a_{1, n}$ os geradores. Na figura 4.10 estão desenhados estes geradores de $\pi_{1}(U)$. O aberto $U \cap V$ tem o mesmo tipo de homotopia que $\mathbb{S}^{1}$. Na figura 4.12 está representado um gerador de $\pi_{1}(U \cap V)$. Notemos que este gerador, incluído em $\pi_{1}(V)$ é igual a $\rho_{1}^{2}$ e incluído em $\pi_{1}(U)$ é igual ao produto de todos os geradores numa certa ordem, que sem perda de generalidade, podemos supor que seja $a_{1,2} \ldots a_{1, n}$. Logo, pelo teorema de Van Kampen, $\pi_{1}\left(F_{n-1,1}\left(\mathbb{R P}^{2}\right)\right)=\left\langle\rho_{1}, a_{1,2}, \ldots, a_{1, n} \mid \rho_{1}^{2}=a_{1,2}, \ldots, a_{1, n}\right\rangle$. Este grupo é livre. De fato, como $n \geq 2$, então $a_{1, n}=a_{1, n-1}^{-1} \ldots a_{1,2}^{-1} \rho_{1}^{2}$, ou seja, podemos retirar $a_{1, n}$ do conjunto de geradores e não existem relações entre os demais geradores.

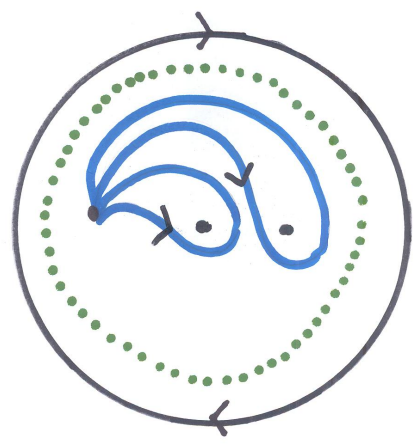

Figura 4.10: aberto $U$

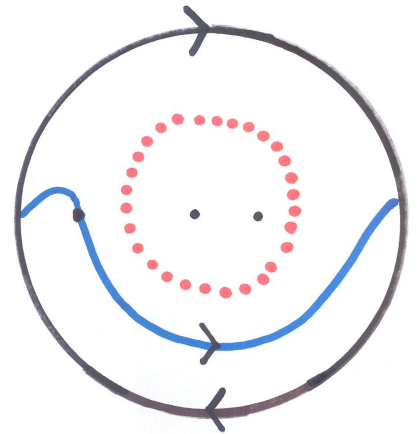

Figura 4.11: aberto $V$

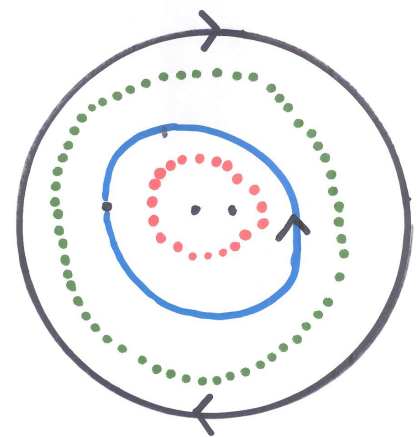

Figura 4.12: $U \cap V$

Como $i_{*}$ é injetiva, podemos considerar, $\pi_{1}\left(F_{n-1,1}\left(\mathbb{R} \mathbb{P}^{2}\right)\right)$ como subgrupo de $P_{n}\left(\mathbb{R P}^{2}\right)$. Notemos que os caminhos $\rho_{1}, a_{1,2}, \ldots, a_{1, n-1}$ do teorema 4.3 .8 em $P_{n}\left(\mathbb{R P}^{2}\right)$ são justamentes as tranças $\rho_{1}, a_{1,2}, \ldots, a_{1, n}$, sendo que estamos usando o mesmo símbolo para as tranças que são imagens por $\tilde{e}_{n}$ das tranças $a_{1,2}, \ldots, a_{1, n} \in P_{n}\left(\mathbb{R}^{2}\right)$. Estas tranças foram definidas no capítulo 3 . Portanto, podemos considerar $\pi_{1}\left(F_{n-1,1}\left(\mathbb{R P}^{2}\right)\right)$ como o subgrupo de $P_{n}\left(\mathbb{R P}^{2}\right)$ gerado por $\rho_{1}, a_{1,2}, \ldots a_{1, n}$ e $i_{*}$ como a inclusão. 
O seguinte resultado nos será útil na seção seguinte e tem uma demonstração que é, salvo poucos detalhes, a mesma do teorema de Fadell-Neuwirth (teorema 1.2.2) e por isso a omitimos.

Teorema 4.3.9. A aplicação $\lambda: \mathcal{A}_{n}\left(\mathbb{S}^{2}\right) \rightarrow \mathcal{A}_{n-1}\left(\mathbb{S}^{2}\right)$, definida por $\lambda\left(x_{1}, x_{2}, \ldots, x_{n}\right)=$ $\left(x_{2}, \ldots, x_{n}\right)$, é uma fibração localmente trivial, com fibra $\mathbb{S}^{2}-Q_{2(n-1)}$, sendo $Q_{2(n-1)}$ um conjunto de $2(n-1)$ pontos distintos e não antipodais de $S^{2}$.

\section{$4.4 \quad 0$ isomorfismo entre $G_{n}\left(\mathbb{R P}^{2}\right)$ e $B_{n}\left(\mathbb{R P}^{2}\right)$}

Consideremos $\phi_{n}: G_{n}\left(\mathbb{R P}^{2}\right) \rightarrow B_{n}\left(\mathbb{R} \mathbb{P}^{2}\right)$ definido na base por

$$
\tilde{\sigma}_{n} \mapsto \sigma_{n} \quad \tilde{\rho}_{n} \mapsto \rho_{n}
$$

e estendido por linearidade. $\phi_{n}$ é um homomorfismo, pois as relações de $G_{n}\left(\mathbb{R P}^{2}\right)$ valem em $B_{n}\left(\mathbb{R P}^{2}\right)$.

O principal objetivo desta seção é provar o seguinte:

Teorema 4.4.1 (principal). Para todo $n, \phi_{n}$ é um isomorfismo.

Notemos que para $n=1$ o teorema é óbvio, desde que $G_{1}\left(\mathbb{R} \mathbb{P}^{2}\right) \approx \mathbb{Z}_{2} \approx B_{1}\left(\mathbb{R} \mathbb{P}^{2}\right)$ e $\phi_{1}$ é o homomorfismo identidade.

Suponhamos então $n \geq 2$. A prova do teorema 4.4.1 será feita por passos, através de várias condições suficientes.

Consideremos $i_{1}: K_{n}\left(\mathbb{R P}^{2}\right) \rightarrow G_{n}\left(\mathbb{R P}^{2}\right)$ e $i_{2}: P_{n}\left(\mathbb{R} \mathbb{P}^{2}\right) \rightarrow B_{n}\left(\mathbb{R P}^{2}\right)$ as inclusões, e $\psi_{n}: K_{n}\left(\mathbb{R P}^{2}\right) \rightarrow P_{n}\left(\mathbb{R P}^{2}\right)$ a restrição de $\phi_{n}$. Então, claramente é comutativo o seguinte diagrama:

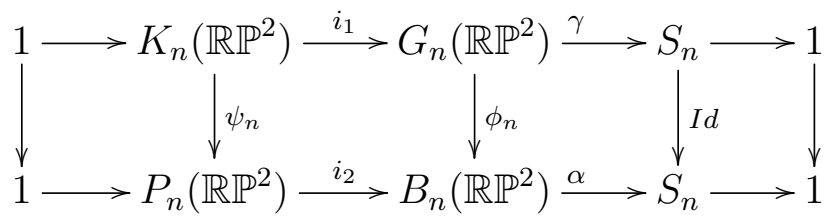

Pelo Lema dos Cinco, temos o:

Lema 4.4.2. Se $\psi_{n}$ é isomorfismo, então $\phi_{n}$ é isomorfismo.

Agora, usando a observação posterior ao teorema 4.3.8, a sequência exata fundamental de $G_{n}\left(\mathbb{R P}^{2}\right)$ (teorema 4.2 .7 ) e a sequência exata curta de Fadell-Neuwirth do $\mathbb{R} \mathbb{P}^{2}$ (definição 4.3.7), temos que é comutativo o seguinte diagrama: 


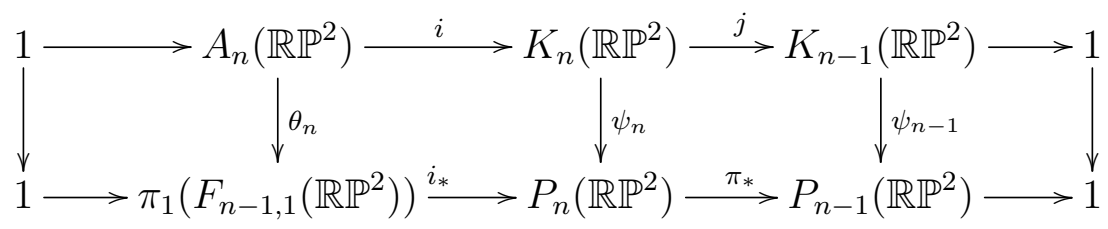

sendo $\theta_{n}: A_{n}\left(\mathbb{R P}^{2}\right) \rightarrow \pi_{1}\left(F_{n-1,1}\left(\mathbb{R P}^{2}\right)\right)$ a restrição do homorfismo $\psi_{n}$.

Como $A_{n}\left(\mathbb{R P}^{2}\right)=\left\langle\tilde{\rho}_{1}, \tilde{a}_{2}, \tilde{a}_{3} \ldots, \tilde{a}_{n} \mid \tilde{\rho}_{1}^{2}=\tilde{a}_{2} \tilde{a}_{3} \ldots \tilde{a}_{n}\right\rangle$ e $\pi_{1}\left(F_{n-1,1}\left(\mathbb{R} \mathbb{P}^{2}\right)\right)=$ $\left\langle\rho_{1}, a_{1,2}, \ldots, a_{1, n} \mid \rho_{1}^{2}=a_{1,2} \ldots a_{1, n}\right\rangle$ pelos teoremas 4.2 .12 e 4.3 .8 e como $\theta_{n}\left(\tilde{\rho}_{1}\right)=\rho_{1}$, $\theta_{n}\left(\tilde{a}_{i}\right)=a_{1, i}$, para todo $i=2, \ldots, n$, então $\theta_{n}$ é um isomorfismo para todo $n \geq 2$. Logo, usando indução e em cada passo indutivo usando o Lema dos Cinco, temos o:

Lema 4.4.3. Se $\psi_{2}$ é isomorfismo, então $\psi_{n}$ é isomorfismo para todo $n \geq 2$.

Consideremos a fibração $\pi: F_{0,2}\left(\mathbb{R P}^{2}\right) \rightarrow F_{0,1}\left(\mathbb{R P}^{2}\right)$, definida no teorema de FadellNeuwirth (teorema 1.2.2), cuja fibra típica neste caso é $F_{1,1}\left(\mathbb{R P}^{2}\right)$.

Notemos que $\pi_{0}\left(F_{1,1}\left(\mathbb{R P}^{2}\right)\right)=1$, já que $\mathbb{R P}^{2}$ menos um ponto é conexo por caminhos. Pelo lema 4.3.6, $\pi_{2}\left(F_{0,2}\left(\mathbb{R P}^{2}\right)\right)=1$. Portanto, a sequência exata de homotopia associada a $\pi$ é a seguinte:

$$
\begin{gathered}
1 \longrightarrow \pi_{2}\left(F_{0,1}\left(\mathbb{R P}^{2}\right), x_{2}\right) \stackrel{\partial}{\longrightarrow} \pi_{1}\left(F_{1,1}\left(\mathbb{R P}^{2}\right), x_{1}\right) \stackrel{i_{*}}{\longrightarrow} \\
\pi_{1}\left(F_{0,2}\left(\mathbb{R P}^{2}\right),\left(x_{1}, x_{2}\right)\right) \stackrel{\pi_{*}}{\longrightarrow} \pi_{1}\left(F_{0,1}\left(\mathbb{R P}^{2}\right), x_{2}\right) \longrightarrow 1
\end{gathered}
$$

$\pi_{1}\left(F_{0,1}\left(\mathbb{R P}^{2}\right), x_{2}\right)=\pi_{1}\left(\mathbb{R P}^{2}\right)$ que é cíclico de ordem 2. Seja $\rho_{2}$ um gerador deste grupo. Como $\mathbb{R P}^{2}$ menos um ponto tem o mesmo tipo de homotopia que $\mathbb{R P}^{1}$, que por sua vez é homeomorfo a $\mathbb{S}^{1}$, então $\pi_{1}\left(F_{1,1}\left(\mathbb{R P}^{2}\right)\right)$ é infinito cíclico. Agora, o recobrimento universal de $\mathbb{R P}^{2}$ é $\mathbb{S}^{2}$. Logo, $\pi_{2}\left(F_{0,1}\left(\mathbb{R P}^{2}\right)\right)=\pi_{2}\left(\mathbb{R P}^{2}\right)=\pi_{2}\left(\mathbb{S}^{2}\right)$ que é ciclíco infinito. Sejam $\rho_{1}$ o gerador de $\pi_{1}\left(F_{1,1}\left(\mathbb{R P}^{2}\right), x_{1}\right)$ e $\omega$ o gerador de $\pi_{2}\left(F_{0,1}\left(\mathbb{R P}^{2}\right), x_{2}\right)$. Suponhamos que $\partial(\omega)=\rho_{1}^{4}$. Então, pelo lema 1.6.2, a seguinte sequência é exata (vamos omitir os pontos bases):

$$
1 \longrightarrow\left\langle\rho_{1} \mid \rho_{1}^{4}=1\right\rangle \stackrel{i_{*}}{\longrightarrow} \pi_{1}\left(F_{0,2}\left(\mathbb{R P}^{2}\right)\right) \stackrel{\pi_{*}}{\longrightarrow}\left\langle\rho_{2} \mid \rho_{2}^{2}=1\right\rangle \longrightarrow 1
$$

Por definição, $K_{1}\left(\mathbb{R P}^{2}\right)=G_{1}\left(\mathbb{R P}^{2}\right)=\left\langle\tilde{\rho}_{2} \mid \tilde{\rho}_{2}^{2}=1\right\rangle$. Pelos teoremas 4.2 .11 e 4.2.9, $A_{2}\left(\mathbb{R} \mathbb{P}^{2}\right)=\left\langle\tilde{\rho}_{1} \mid \tilde{\rho}_{1}^{4}=1\right\rangle, K_{2}\left(\mathbb{R} \mathbb{P}^{2}\right)=\left\langle\tilde{\rho}_{1}, \tilde{\rho}_{2} \mid\left(\tilde{\rho}_{2} \tilde{\rho}_{1}\right)^{2}=\tilde{\rho}_{1}^{2}=\tilde{\rho}_{2}^{2}\right\rangle$. Usando a sequência exata fundamental de $G_{2}\left(\mathbb{R P}^{2}\right)$, temos a seguinte sequência exata:

$$
1 \longrightarrow A_{2}\left(\mathbb{R P}^{2}\right) \stackrel{i}{\longrightarrow} K_{2}\left(\mathbb{R P}^{2}\right) \stackrel{j}{\longrightarrow} K_{1}\left(\mathbb{R P}^{2}\right) \longrightarrow 1
$$


sendo $i: A_{2}\left(\mathbb{R P}^{2}\right) \rightarrow K_{2}\left(\mathbb{R P}^{2}\right)$ definido na base por $i\left(\tilde{\rho}_{1}\right)=\tilde{\rho}_{1}$ e $j: K_{2}\left(\mathbb{R P}^{2}\right) \rightarrow$ $K_{1}\left(\mathbb{R P}^{2}\right)$ definido na base por $j\left(\tilde{\rho}_{1}\right)=1$ e $j\left(\tilde{\rho}_{2}\right)=\tilde{\rho}_{2}$.

Notemos que $\psi_{1}: K_{1}\left(\mathbb{R P}^{2}\right) \rightarrow \pi_{1}\left(F_{0,1}\left(\mathbb{R P}^{2}\right)\right)$ é isomorfismo. Se definirmos $\theta_{2}$ : $A_{2}\left(\mathbb{R P}^{2}\right) \rightarrow\left\langle\rho_{1} \mid \rho_{1}^{4}=1\right\rangle$ por $\theta_{2}\left(\tilde{\rho}_{1}\right)=\rho_{1}$, então $\theta_{2}$ é isomorfismo e o seguinte diagrama é comutativo, cujas sequências na horizantal são exatas:

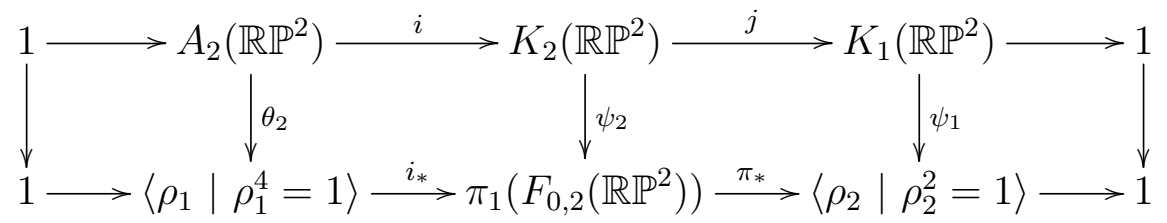

Pelo lema dos cinco, seguirá que $\psi_{2}$ é isomorfismo. Portanto, para que fique provado o teorema 4.4.1, o restante desta seção será dedicada a provar o

Lema 4.4.4. $\partial$ é a multiplicação por 4.

Prova: Nesta demonstração, vamos considerar $\mathbb{R P}^{2}$ como o espaço quociente de $\mathbb{S}^{2}$, onde se identificam os pontos antípodas. Pelo teorema 4.3.9, a aplicação $\lambda:\left(\mathcal{A}_{2}\left(\mathbb{S}^{2}\right),\left(x_{1}^{0}, x_{2}^{0}\right)\right) \rightarrow\left(\mathbb{S}^{2}, x_{2}^{0}\right)$, dada por $\lambda\left(x_{1}, x_{2}\right)=x_{2}$, é uma fibração localmente trivial com fibra típica $\mathbb{S}^{2}-\left\{ \pm x_{2}^{0}\right\}$. Pelo teorema 4.3.2, $\mathcal{A}_{2}\left(\mathbb{S}^{2}\right)$ recobre $F_{0,2}\left(\mathbb{R P}^{2}\right)$ e como $\pi_{2}\left(F_{0,2}\left(\mathbb{R P}^{2}\right)\right)=1$ pelo lema 4.3 .6 , então $\pi_{2}\left(\mathcal{A}_{2}\left(\mathbb{S}^{2}\right)\right)=1$. Como $\pi_{1}\left(\mathbb{S}^{2}\right)=1$, então a sequência exata de homotopia da fibração $\lambda$ nos fornece:

$$
1 \longrightarrow \pi_{2}\left(\mathbb{S}^{2}, x_{2}^{0}\right) \stackrel{\bar{\partial}}{\longrightarrow} \pi_{1}\left(\mathbb{S}^{2}-\left\{ \pm x_{2}^{0}\right\}, x_{1}^{0}\right) \stackrel{i_{*}^{\prime}}{\longrightarrow} \pi_{1}\left(\mathcal{A}_{2}\left(\mathbb{S}^{2}\right),\left(x_{1}^{0}, x_{2}^{0}\right)\right) \longrightarrow 1
$$

sendo $i_{*}^{\prime}$ a induzida da aplicação $i^{\prime}:\left(\mathbb{S}^{2}-\left\{ \pm x_{2}^{0}\right\}, x_{1}^{0}\right) \rightarrow\left(\mathcal{A}_{2}\left(\mathbb{S}^{2}\right),\left(x_{1}^{0}, x_{2}^{0}\right)\right)$, definida por $i^{\prime}\left(x_{1}\right)=\left(x_{1}, x_{2}^{0}\right) \cdot \pi_{2}\left(\mathbb{S}^{2}\right)$ é cíclico de ordem infinita. Pela projeção estereográfica, $\mathbb{S}^{2}- \pm x_{2}^{0}$ é homeomorfo a $\mathbb{R}^{2}$ menos um ponto. Logo, $\pi_{1}\left(\mathbb{S}^{2}- \pm x_{2}^{0}\right)$ é cíclico de ordem infinita. Em [12], encontramos uma prova que $\pi_{1}\left(V_{3,2}\right)$ é cíclico de ordem 2 e como $\mathcal{A}_{2}\left(\mathbb{S}^{2}\right)$ tem o mesmo tipo de homotopia que $V_{3,2}$, de acordo com o lema 4.3 .3 , então $\pi_{1}\left(\mathcal{A}_{2}\left(\mathbb{S}^{2}\right)\right)$ é cíclico de ordem 2. Logo, $\bar{\partial}$ é a multiplicação por 2. Voltemos a considerar a fibração $\pi:\left(F_{0,2}\left(\mathbb{R P}^{2}\right),\left(\left[x_{1}^{0}\right],\left[x_{2}^{0}\right]\right)\right) \rightarrow\left(F_{0,1}\left(\mathbb{R P}^{2}\right),\left[x_{2}^{0}\right]\right)$, cuja fibra típica é $\mathbb{R P}^{2}-\left[x_{2}^{0}\right]$. Seja $\tilde{p}: \mathbb{S}^{2} \rightarrow \mathbb{R P}^{2}$ a projeção natural. Notemos que é comutativo o seguinte diagrama:

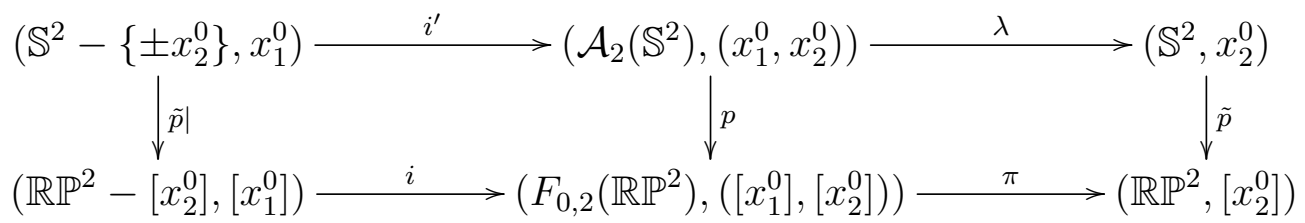

sendo $p$ o recobrimento definido no teorema 4.3 .2 e $\tilde{p} \mid$ a restrição de $\tilde{p}$. Pela naturalidade da sequência exata de homotopia, temos o seguinte diagrama comutativo (omitimos os 
pontos base e só exibimos a parte que nos interessa):

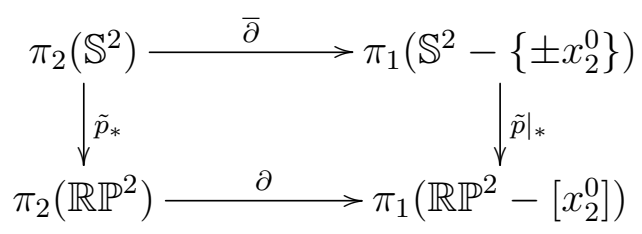

Notemos que $\mathbb{R P}^{2}-\left[x_{2}^{0}\right]$ tem o mesmo tipo de homotopia que $\mathbb{R P}^{1}$. Agora $\mathbb{R P}^{1}$ é homeomorfo a $\mathbb{S}^{1}$. Logo, $\pi_{1}\left(\mathbb{R P}^{2}-\left[x_{2}^{0}\right]\right)$ é cíclico de ordem infinita. Podemos, sem perda de generalidade, considerar $\pm x_{2}^{0}$ como sendo o polo norte e sul de $\mathbb{S}^{2}$. Um gerador de $\pi_{1}\left(\mathbb{S}^{2}-\left\{ \pm x_{2}^{0}\right\}\right)$ é um loop que sai do ponto $(1,0,0)$ e dá a volta pelo equador. Notemos que, pela definição de $\tilde{p}, \tilde{p} \mid *$ aplicado neste gerador dá duas voltas em $\pi_{1}\left(\mathbb{R P}^{2},\left[x_{2}^{0}\right]\right)$. Logo, $\left.\tilde{p}\right|_{*}$ é a multiplicação por 2. Como $\tilde{p}$ é recobrimento, então $\tilde{p}_{*}$ é isomorfismo, e portanto, $\partial=\left(\left.\tilde{p}\right|_{*}\right) \circ \bar{\partial} \circ\left(\tilde{p}_{*}\right)^{-1}$ é a multiplicação por 4 . 


\section{Grupos de Tranças Puras do Espaço \\ Projetivo}

Assim como as tranças de Artin não geram $B_{n}\left(\mathbb{R P}^{2}\right)$, as tranças puras $a_{i, j}, 1 \leq i<j \leq n$, que foram definidas no capítulo 3 , pensadas como imagem do homomorfismo $\tilde{e}_{n}$, não geram todo $P_{n}\left(\mathbb{R P}^{2}\right)$.

Consideremos em $\mathbb{R P}^{2}$ um caminho injetor $\bar{\xi}_{k}$ conforme a descrição da figura 5.1. Na figura, $n=5$ e $k=3$.

Definimos a trança $\xi_{k}=\left(b_{1}, \ldots, b_{n}\right) \in P_{n}\left(\mathbb{R P}^{2}\right)$, cujas cordas são os caminhos que tem a seguinte expressão:

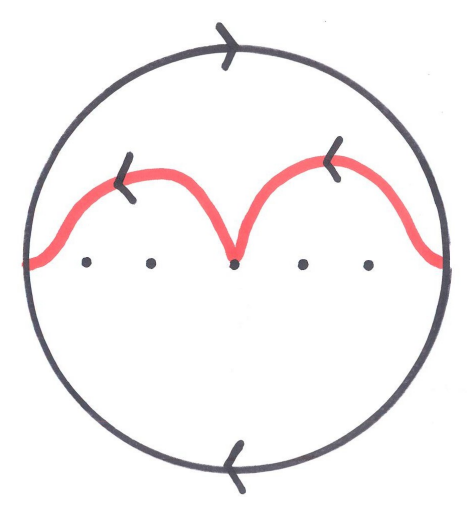

$$
\xi_{j}(t)=(e(j, 0), t), j \neq k \quad \xi_{k}(t)=\left(\bar{\xi}_{k}(t), t\right)
$$

$\mathrm{Na}$ figura 5.2 estão representados os esquemas das Figura 5.1: caminho $\overline{\xi_{k}}$ tranças $\xi_{k}$ e $\xi_{k}^{-1}$ quando $n=5$ e $k=3$.

Como as tranças $a_{i, j}$ são imagem do homomorfismo $\tilde{e}_{n}$, valem as relações do plano (a) $a_{r, s} a_{i, j} a_{r, s}^{-1}=\left\{\begin{array}{l}a_{i, j}, \text { se } i<r<s<j \text { ou } r<s<i<j \\ a_{i, j}^{-1} a_{r, j}^{-1} a_{i, j} a_{r, j} a_{i, j}, \text { se } r<i=s<j \\ a_{s, j}^{-1} a_{i, j} a_{s, j}, \text { se } i=r<s<j \\ a_{s, j}^{-1} a_{r, j}^{-1} a_{s, j} a_{r, j} a_{i, j} a_{r, j}^{-1} a_{s, j}^{-1} a_{r, j} a_{s, j}, \text { se } r<i<s<j\end{array}\right.$ 

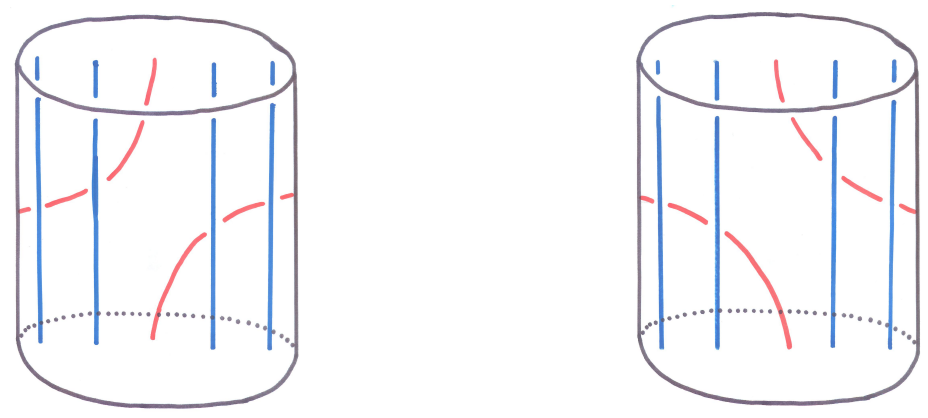

Figura 5.2: tranças $\xi_{k}$ e $\xi_{k}^{-1}$
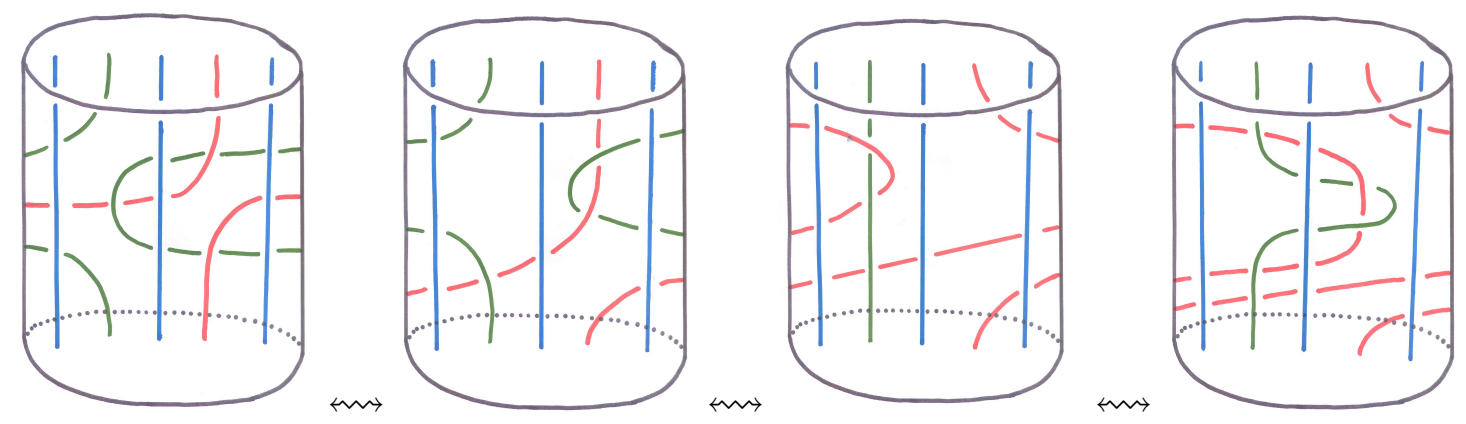

Figura 5.3: relação $(b)$
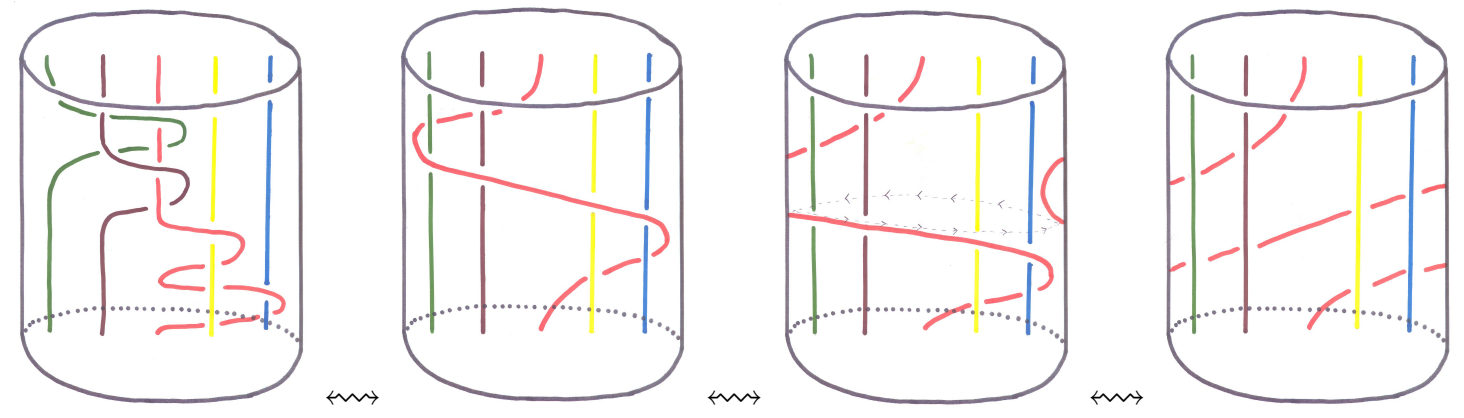

Figura 5.4: relação $(c)$
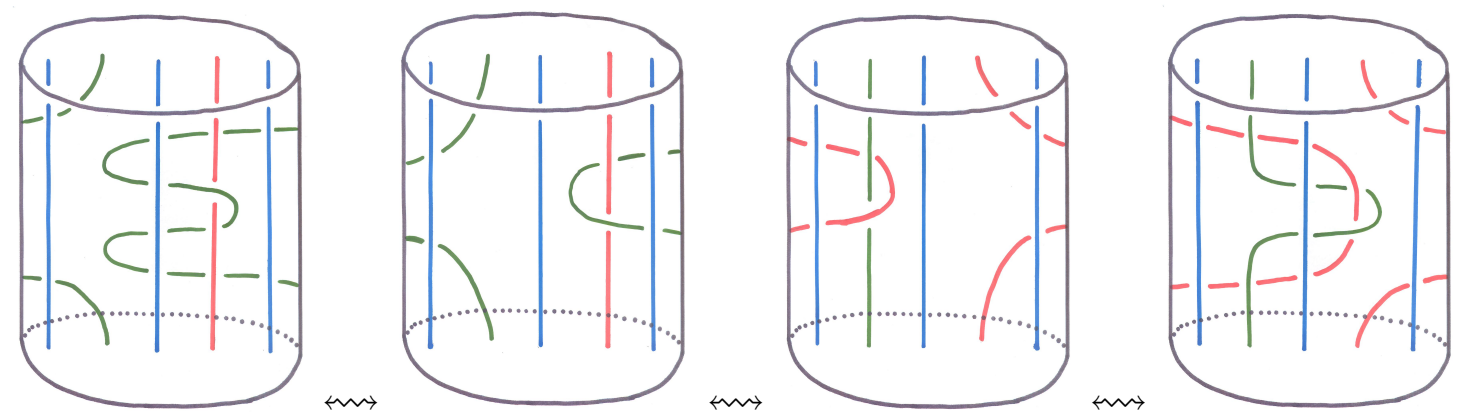

Figura 5.5: relação $(d)$ 


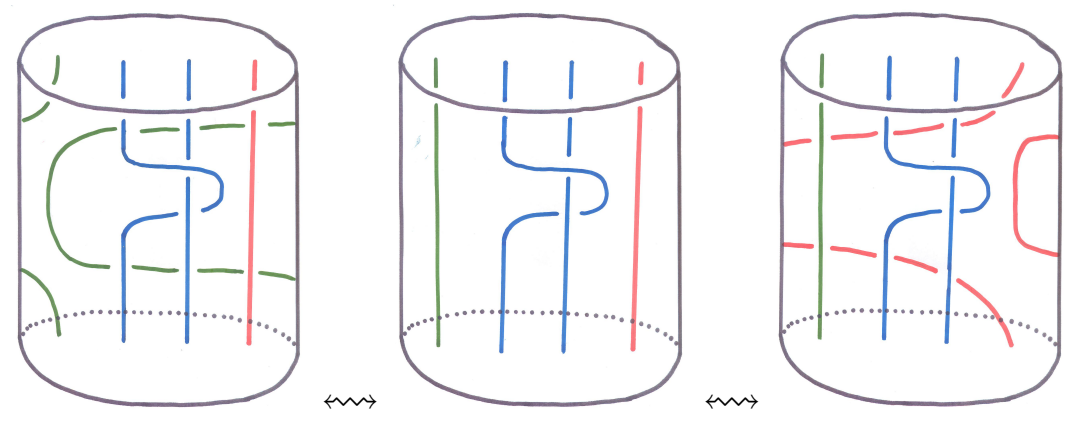

Figura 5.6: relação $(e)$

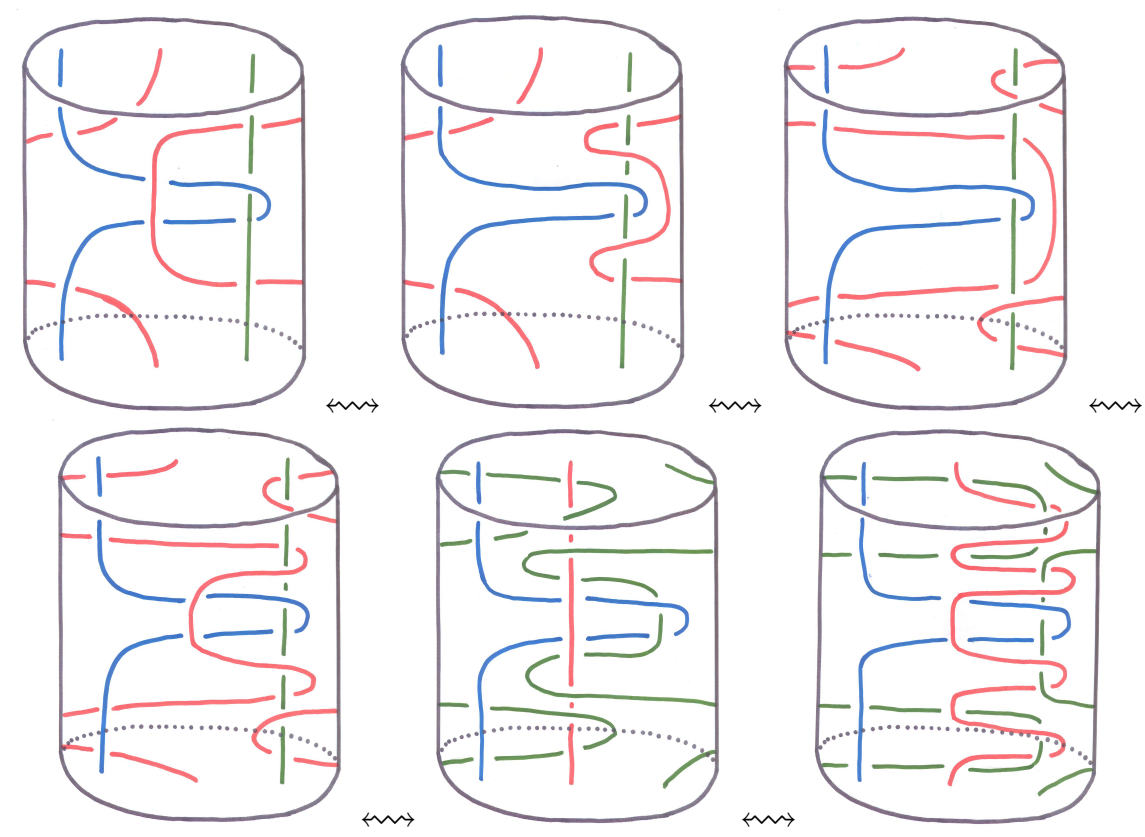

Figura 5.7: relação $(f)$

As figuras 5.3, 5.4, 5.5, 5.6 e 5.7 mostram que as tranças $\xi_{k}, 1 \leq k \leq n$, junto com as tranças $a_{i, j}, 1 \leq i<j \leq n$, estão sujeitas as seguintes relações:

(b) $\xi_{i} \xi_{j} \xi_{i}^{-1}=\xi_{j}^{-1} a_{i, j}^{-1} \xi_{j}^{2}$

(c) $\xi_{i}^{2}=a_{1, i} \ldots a_{i-1, i} a_{i, i+1} \ldots a_{i, n}$;

(d) $\xi_{i} a_{i, j} \xi_{i}^{-1}=\xi_{j}^{-1} a_{i, j}^{-1} \xi_{j}$;

(e) $\xi_{k} a_{i, j} \xi_{k}^{-1}=a_{i, j}$, se $i<j<k$ ou $k<i<j$;

(f) $\xi_{k} a_{i, j} \xi_{k}^{-1}=\xi_{j}^{-1} a_{k, j}^{-1} \xi_{j} a_{k, j}^{-1} a_{i, j} a_{k, j} \xi_{j}^{-1} a_{k, j} \xi_{j}$, se $i<k<j$. 
O objetivo deste capítulo é mostrar que as tranças $\xi_{k}, 1 \leq k \leq n$, e $a_{i, j}, 1 \leq i<$ $j \leq n$, geram $P_{n}\left(\mathbb{R P}^{2}\right)$ e que as relações $(a),(b),(c),(d),(e)$ e $(f)$ formam um sistema completo de relações para $P_{n}\left(\mathbb{R P}^{2}\right)$, isto é, qualquer outra relação é proveniente destas.

Consideremos $\tilde{G}_{n}$ o grupo cuja apresentação é a apresentação que queremos obter para $P_{n}\left(\mathbb{R P}^{2}\right)$. Vamos mostrar que $\tilde{G}_{n}$ é isomorfo a $P_{n}\left(\mathbb{R P}^{2}\right)$ através de 3 teoremas, que juntos, usando indução, provam ser verdadeira a apresentação que queremos obter.

Teorema 5.0.5. $\tilde{G}_{1}$ é isomorfo a $P_{1}\left(\mathbb{R} \mathbb{P}^{2}\right)$.

Prova: Como $P_{1}\left(\mathbb{R P}^{2}\right)=\pi_{1}\left(\mathbb{R P}^{2}\right) \approx \mathbb{Z}_{2}$, basta mostrar que $\tilde{G}_{1}$ é cíclico de ordem 2 . Mas isto segue direto da definição, pois em $\tilde{G}_{1}$ só existe um gerador, $\xi_{1}$, e exceto a relação $(c) \xi_{1}^{2}=1$, todas as outras são relações triviais.

Teorema 5.0.6. $\tilde{G}_{2}$ é isomorfo a $P_{2}\left(\mathbb{R} \mathbb{P}^{2}\right)$.

Prova: Como corolário da demonstração do teorema 4.4.1 e pelo teorema 4.2.9, $P_{2}\left(\mathbb{R P}^{2}\right)$ é isomorfo ao grupo dos quatérnios. Portanto, para mostrar o teorema, basta mostrarmos que $\tilde{G}_{2}$ é isomorfo a $D i c_{8}$, pois de acordo com a seção 1.5, o grupo dicíclico de ordem 8 é isomorfo ao grupo dos quatérnios. Por definição, $\tilde{G}_{2}$ é gerado por $\xi_{1}, \xi_{2}, a_{1,2}$, sujeito as seguintes relações:
(b) $\xi_{1} \xi_{2} \xi_{1}^{-1}=\xi_{2}^{-1} a_{1,2}^{-1} \xi_{2}^{2}$
(c) $\xi_{1}^{2}=\xi_{2}^{2}=a_{1,2}$
(d) $\xi_{1} a_{1,2} \xi_{1}^{-1}=\xi_{2}^{-1} a_{1,2}^{-1} \xi_{2}$

Por $(c)$, podemos tomar $\xi_{2}^{2}$ como definição de $a_{1,2}$ e usando a relação $(c)$ nas relações (b) e $(d)$, temos que $\tilde{G}_{2}$ é gerado por $\xi_{1}$ e $\xi_{2}$, com as seguintes relações:

$$
\left(b^{\prime}\right) \xi_{1} \xi_{2} \xi_{1}^{-1}=\xi_{2}^{-1} \quad\left(c^{\prime}\right) \xi_{1}^{2}=\xi_{2}^{2} \quad\left(d^{\prime}\right) \xi_{1}^{2}=\xi_{2}^{-2}
$$

Notemos que $\left(d^{\prime}\right) \stackrel{\left(c^{\prime}\right)}{\Longleftrightarrow} \xi_{1}^{2}=\xi_{1}^{-2} \Longleftrightarrow \xi_{1}^{4}=1$.

Notemos ainda que $\xi_{2}^{3}=\xi_{2}^{3} \Longleftrightarrow \xi_{2}^{2} \xi_{2}=\xi_{2} \xi_{2}^{2} \stackrel{\left(c^{\prime}\right)}{\Longleftrightarrow} \xi_{1}^{2} \xi_{2}=\xi_{2} \xi_{1}^{2} \Longleftrightarrow \xi_{1} \xi_{2} \xi_{1}^{-1}=$ $\xi_{1}^{-1} \xi_{2} \xi_{1}$.

Logo, a relação $\left(b^{\prime}\right)$ é equivalente a relação $\xi_{1}^{-1} \xi_{2} \xi_{1}=\xi_{2}^{-1}$.

Portanto,

$$
\tilde{G}_{2}=<\xi_{1}, \xi_{2} \mid \xi_{1}^{4}=1, \xi_{1}^{2}=\xi_{2}^{2}, \xi_{1}^{-1} \xi_{2} \xi_{1}=\xi_{2}^{-1}>
$$

que é uma apresentação para $D i c_{8}$, conforme mostrado na seção 1.5.

No capítulo anterior, usando a fibração localmente trivial $\pi$, definida no teorema de Fadell e Neuwirth (teorema 1.2.2), definimos, para $n \geq 3$, a sequência exata curta de Fadell-Neuwirth

$$
1 \longrightarrow \pi_{1}\left(F_{n-1,1}\left(\mathbb{R} \mathbb{P}^{2}\right)\right) \stackrel{i_{*}}{\longrightarrow} P_{n}\left(\mathbb{R P}^{2}\right) \stackrel{\pi_{*}}{\longrightarrow} P_{n-1}\left(\mathbb{R P}^{2}\right) \longrightarrow 1
$$


e observamos que $\pi_{1}\left(F_{n-1,1}\left(\mathbb{R} \mathbb{P}^{2}\right)\right)$ podia ser considerado como subgrupo de $P_{n}\left(\mathbb{R P}^{2}\right)$ gerado por $\rho_{1}, a_{1,2} \ldots a_{1, n}, i_{*}$ o homomorfismo inclusão e $\pi_{*}$ era o homomorfismo que retirava a primeira corda.

Agora, usando a fibração $\epsilon$ do teorema de Fadell e Neuwirth, de maneira totalmente análoga, podemos mostrar que $\pi_{1}\left(F_{n-1,1}\left(\mathbb{R P}^{2}\right)\right)$ pode ser considerado como subgrupo de $P_{n}\left(\mathbb{R P}^{2}\right)$ gerado por $\xi_{n}, a_{1, n}, \ldots, a_{n-1, n}$ com a relação $\xi_{n}^{2}=a_{1, n} \ldots a_{n-1, n}$ e definirmos, para $n \geq 3$ a sequência de Fadell-Neuwirth

$$
1 \longrightarrow \pi_{1}\left(F_{n-1,1}\left(\mathbb{R P}^{2}\right)\right) \stackrel{i_{*}}{\longrightarrow} P_{n}\left(\mathbb{R P}^{2}\right) \stackrel{\epsilon_{*}}{\longrightarrow} P_{n-1}\left(\mathbb{R P}^{2}\right) \longrightarrow 1
$$

Novamente, $i_{*}$ pode ser considerado como a inclusão, mas agora, o homomorfismo $\epsilon_{*}$ corresponde a retirar a última corda de uma trança.

Usaremos esses fatos para provar o

Teorema 5.0.7. Se $n \geq 2$ e $\tilde{G}_{n}$ é isomorfo a $P_{n}\left(\mathbb{R P}^{2}\right)$, então $\tilde{G}_{n+1}$ é isomorfo a $P_{n+1}\left(\mathbb{R P}^{2}\right)$.

Prova: Como $n \geq 2$, temos a sequência de Fadell - Neuwirth

$$
1 \longrightarrow \pi_{1}\left(F_{n, 1}\left(\mathbb{R P}^{2}\right)\right) \stackrel{i_{*}}{\longrightarrow} P_{n+1}\left(\mathbb{R P}^{2}\right) \stackrel{\epsilon_{*}}{\longrightarrow} P_{n}\left(\mathbb{R P}^{2}\right) \longrightarrow 1
$$

Lembrando que $\pi_{1}\left(F_{n, 1}\left(\mathbb{R P}^{2}\right)\right)=<\xi_{n+1}, a_{1, n+1}, \ldots, a_{n, n+1} \mid \xi_{n+1}^{2}=a_{1, n+1} \ldots a_{n, n+1}>$ e como, por hipótese já temos a apresentação de $P_{n}\left(\mathbb{R P}^{2}\right)$, então podemos aplicar o método de apresentação de uma extensão. Pelo método, $P_{n+1}\left(\mathbb{R P}^{2}\right)$ é gerado por elementos que são mapeados por $\epsilon_{*}$ nos geradores de $P_{n}\left(\mathbb{R P}^{2}\right)$ e também pelos geradores de $\pi_{1}\left(F_{n, 1}\left(\mathbb{R P}^{2}\right)\right)$. Notemos que $\epsilon_{*}\left(\xi_{k}\right)=\xi_{k}$, se $1 \leq k \leq n$, e $\epsilon_{*}\left(a_{i, j}\right)=a_{i, j}$, se $1 \leq i<j \leq n$, já que $\epsilon_{*}$ é o homomorfismo que corresponde a retirar a última corda de uma trança. Logo,

$$
P_{n+1}\left(\mathbb{R P}^{2}\right) \text { é gerado por } \xi_{k}, 1 \leq k \leq n+1 \text { e } a_{i, j}, 1 \leq i<j \leq n+1 \text {. }
$$

Agora, pelo método, temos que calcular 3 classes de relações. A primeira é a inclusão das relações de $\pi_{1}\left(F_{n, 1}\left(\mathbb{R P}^{2}\right)\right)$. Como só ha uma relação, temos que em $P_{n+1}\left(\mathbb{R P}^{2}\right)$ vale

$$
\xi_{n+1}^{2}=a_{1, n+1} \ldots a_{n, n+1} .
$$

A segunda classe de relações é obtida da seguinte maneira: consideremos cada relação de $P_{n}\left(\mathbb{R P}^{2}\right)$ com um elemento $P_{n+1}\left(\mathbb{R P}^{2}\right)$. O método garante que existe um elemento de $\pi_{1}\left(F_{n, 1}\left(\mathbb{R P}^{2}\right)\right)$ que é igual a relação que estamos considerando e esta igualdade é uma relação de $P_{n+1}\left(\mathbb{R P}^{2}\right)$. Exceto a relação $(c)$, já mostramos que todas as outras relações valem em $P_{n+1}\left(\mathbb{R P}^{2}\right)$. Logo, podemos tomar o elemento neutro de $\pi_{1}\left(F_{n, 1}\left(\mathbb{R P}^{2}\right)\right.$ para igualar as estas relações e assim, temos que em $P_{n+1}\left(\mathbb{R} \mathbb{P}^{2}\right)$ vale 
$a_{r, s} a_{i, j} a_{r, s}^{-1}=\left\{\begin{array}{l}a_{i, j}, \text { se } 1 \leq i<r<s<j \leq n \text { ou } 1 \leq r<s<i<j \leq n \\ a_{i, j}^{-1} a_{r, j}^{-1} a_{i, j} a_{r, j} a_{i, j}, \text { se } 1 \leq r<i=s<j \leq n \\ a_{s, j}^{-1} a_{i, j} a_{s, j}, \text { se } 1 \leq i=r<s<j \leq n \\ a_{s, j}^{-1} a_{r, j}^{-1} a_{s, j} a_{r, j} a_{i, j} a_{r, j}^{-1} a_{s, j}^{-1} a_{r, j} a_{s, j}, \text { se } 1 \leq r<i<s<j \leq n\end{array}\right.$

e para $1 \leq i<j \leq n$ e $1 \leq k \leq n$ :

$\xi_{i} \xi_{j} \xi_{i}^{-1}=\xi_{j}^{-1} a_{i, j}^{-1} \xi_{j}^{2}$

$\xi_{i} a_{i, j} \xi_{i}^{-1}=\xi_{j}^{-1} a_{i, j}^{-1} \xi_{j}$;

$\xi_{k} a_{i, j} \xi_{k}^{-1}=a_{i, j}$, se $i<j<k$ ou $k<i<j$;

$\xi_{k} a_{i, j} \xi_{k}^{-1}=\xi_{j}^{-1} a_{k, j}^{-1} \xi_{j} a_{k, j}^{-1} a_{i, j} a_{k, j} \xi_{j}^{-1} a_{k, j} \xi_{j}$, se $i<k<j$.

Agora, já mostramos que em $P_{n+1}\left(\mathbb{R P}^{2}\right)$ vale $\xi_{i}^{2}=a_{1, i} \ldots a_{i-1, i} a_{i, i+1} \ldots a_{i, n} a_{i, n+1}$ e como $\xi_{i}^{2}=a_{1, i} \ldots a_{i-1, i} a_{i, i+1} \ldots a_{i, n}$ é uma relação de $P_{n}\left(\mathbb{R} \mathbb{P}^{2}\right)$ e $a_{i, n+1} \in \pi_{1}\left(F_{n, 1}\left(\mathbb{R} \mathbb{P}^{2}\right)\right)$, então, pelo método, precisamos por em $P_{n+1}\left(\mathbb{R P}^{2}\right)$ a relação

$$
\xi_{i}^{2}=a_{1, i} \ldots a_{i-1, i} a_{i, i+1} \ldots a_{i, n} a_{i, n+1}, 1 \leq i \leq n .
$$

A terceira classe de relações é obtida conjugando cada gerador de $\pi_{1}\left(F_{n, 1}\left(\mathbb{R P}^{2}\right)\right)$ por cada gerador de $P_{n}\left(\mathbb{R P}^{2}\right)$ e pelo método, esta conjugação será um elemento de $\pi_{1}\left(F_{n, 1}\left(\mathbb{R P}^{2}\right)\right)$. Novamente, usando as relações que já provamos e a sequência de Fadell-Neuwirth para garantir junto com o método a veracidade das relações, temos:

Para todo $1 \leq i<j \leq n$, para todo $1 \leq k \leq n$ e para todo $1 \leq l \leq n$

$$
\begin{aligned}
& a_{i, j} a_{l, n+1} a_{i, j}^{-1}=\left\{\begin{array}{l}
a_{l, n+1}, \text { se } l<i \text { ou } j<l \\
a_{l, n+1}^{-1} a_{i, n+1}^{-1} a_{l, n+1} a_{i, n+1} a_{l, n+1}, \text { se } l=j \\
a_{j, n+1}^{-1} a_{l, n+1} a_{j, n+1}, \text { se } l=i \\
a_{j, n+1}^{-1} a_{i, n+1}^{-1} a_{j, n+1} a_{i, n+1} a_{l, n+1} a_{i, n+1}^{-1} a_{j, n+1}^{-1} a_{r, j} a_{i, n+1}, \text { se } i<l<j
\end{array}\right. \\
& a_{i, j}=\xi_{n+1} a_{i, j}^{-1}=\xi_{n+1} \\
& \xi_{k} \xi_{n+1} \xi_{k}^{-1}=\xi_{n+1}^{-1} a_{k, n+1}^{-1} \xi_{n+1}^{2} \\
& \xi_{k} a_{l, n+1} \xi_{k}^{-1}\left\{\begin{array}{l}
a_{l, n+1}, \text { se } k<l \\
\xi_{n+1}^{-1} a_{l, n+1}^{-1} \xi_{n+1}, \text { se } k=l \\
\xi_{n+1}^{-1} a_{k, n+1}^{-1} \xi_{n+1} a_{k, n+1}^{-1} a_{k, n+l} a_{k, n+l} \xi_{n+1}^{-1} a_{k, n+1} \xi_{n+1} \text { se } l<k
\end{array}\right.
\end{aligned}
$$

Combinando todos os índices, obtemos a apresentação de $\tilde{G}_{n+1}$, e portanto, $\tilde{G}_{n+1}$ é isomomorfo a $P_{n+1}\left(\mathbb{R P}^{2}\right)$. 


\section{Referências Bibliográficas}

[1] E. Artin, Theorie der Zöpfe, Abhandlungen aus dem Mathematischen Seminar der Universität Hamburg4, 47-72, 1925

[2] E. Artin, Theory of Braids, Annals of Mathematics 48, 101-126, 1947

[3] E. Artin, Braids and permutations, Annals of Mathematics 48, 643-648, 1947

[4] R. H. Fox, L. Neuwirth, The braid groups, Mathematica Scandinavica 10, 119126, 1962

[5] J . S. Birman, On braids groups, Communications on Pure and Applied Mathematics 22, 643-649, 1947

[6] J. S. Birman, Braids, Links, and Mapping Class Groups, Annals of Mathematics Studies 82, Princeton University Press, 1974

[7] V. L. Hansen, Braids and Coverings: selectd topics, London Mathematical Society Student Texts 18, Cambridge University Press, 1989

[8] K. Murasugi, B. I. Kurpita, A Study of Braids, Mathematics and Its Aplications 484, Kluwer Academic Publishers, 1999

[9] J. V. Buskirk, Braid Groups os Compact 2-Manifolds with Elements of Finite Order, Transactions of the American Mathematical Society 122 81-97, 1966

[10] D. L. Gonçalves, J. Guaschi, The braid group of the projective plane and the Fadell-Neuwirth short exact sequence, Geometria Dedicata 130 93-107, 2007

[11] W. Magnus, A. Karras, D. Solitar, Combinatorial Group Theory: Presentations of Groups in terms of generators and relations, Interscience Publishers, 1966 
[12] G. W. Whitehead, Elements of homotopy theory, Graduate Texts in Mathematics 61, Springer - Verlag, 1978

[13] E. L. Lima, Grupo Fundamental e Espaços de Recobrimento, Projeto Euclides, IMPA, 2006

[14] P. J. Hilton, An Introduction to Homotopy Theory, Cambridge at The University Press, 1964

[15] D. L. Johnson, Presentatios of groups, London Mathematical Society Lecture Notes Series 22, Cambridge University Press, 1976

[16] E. Fadell, J. Van Buskirk, The braid groups of $E^{2}$ and $S^{2}$, Duke Mathematical Journal 29, 243-258, 1962

[17] H. S. M. Coxeter, W. O. J. Moser, Generators and relations for discrete groups, Ergebnisse Series, Berlim, 1957

[18] M. P. do Carmo, Geometria Diferencial de Curvas e Superfícies, Textos Universitários, Sociedade Brasileira de Matemática, 2005

[19] A. Garcia, Y. Lequain, Elementos de Álgebra, Projeto Euclides, IMPA, 2006

[20] N. E. Steenrod, The topology of fibre bundles, Princeton mathematical series 14, Princeton University Press, 1951

[21] D. Husemoller, Fibre bundles, Graduate texts in mathematics 20, Springer Verlag, 1994

[22] G. F. Paechter, The groups $\pi_{r}\left(V_{n, m}\right)$. I, The Quartely Journal of Mathematics, 7, 249-268, 1956 